\title{
BOW SHOCK AND UPSTREAM PHENOMENA AT MARS
}

\author{
C. MAZELLE ${ }^{1}$, D. WINTERHALTER ${ }^{2}$, K. SAUER $^{3}$, J.G. TROTIGNON ${ }^{4}$, \\ M.H. ACUÑA ${ }^{5}$, K. BAUMGÄRTEL ${ }^{6}$, C. BERTUCCI ${ }^{1}$, D.A. BRAIN ${ }^{7}$, S.H. BRECHT $^{8}$, \\ M. DELVA ${ }^{9}$, E. DUBININ ${ }^{3}$, M. ØIEROSET ${ }^{10}$ and J. SLAVIN $^{5}$ \\ ${ }^{1}$ C.E.S.R., CNRS/UPS, 9-11 Avenue du Colonel Roche, 31400 Toulouse, France \\ ${ }^{2}$ Jet Propulsion Laboratory, California Institute of Technology, Pasadena, CA, U.S.A. \\ ${ }^{3}$ Max-Planck-Institut für Aeronomie, Katlenburg-Lindau, Germany \\ ${ }^{4}$ L.P.C.E., CNRS, Orléans, France \\ ${ }^{5}$ NASA Goddard Space Flight Center, Greenbelt, MD 20771, U.S.A. \\ ${ }^{6}$ Astrophysikalisches Institut Potsdam, Potsdam, Germany \\ ${ }^{7}$ Department of Astrophysical and Planetary Sciences, University of Colorado, Boulder, CO, U.S.A. \\ ${ }^{8}$ Berkeley Research Associates, Berkeley, CA, U.S.A. \\ ${ }^{9}$ Institut für Weltraumforschung, Graz, Austria \\ ${ }^{10}$ Space Science Laboratory, University of California, Berkeley, CA, U.S.A.
}

(Accepted in final form 14 March 2003)

\begin{abstract}
Mars Global Surveyor is the sixth spacecraft to return measurements of the Martian bow shock. The earlier missions were Mariner 4 (1964), Mars 2 and 3 (1972), Mars 5 (1975) and Phobos 2 (1989) (see reviews by Gringauz, 1981; Slavin and Holzer, 1982; Russell, 1985; Vaisberg, 1992a,b; Zakharov, 1992). Previous investigations of planetary bow shocks have established that their position, shape and jump conditions are functions of the upstream flow parameters and the nature of the solar wind - planet interaction (Spreiter and Stahara, 1980; Slavin et al., 1983; Russell, 1985). At Mars, however, the exact nature of the solar wind interaction was elusive due to the lack of low altitude plasma and magnetic field measurements (e.g., Axford, 1991). In fact our knowledge of the nature of the interaction of Mars with the solar wind was incomplete until the arrival of MGS and the acquisition of close-in magnetic field data (Acuña et al., 1998). As detailed by a series of review papers in this monograph, the Mars Global Surveyor (MGS) mission has now shown that the Mars environment is very complex with strong, highly structured crustal magnetic remnants in the southern hemisphere, while the northern hemisphere experiences the direct impingement of solar wind plasma. This review paper first presents a survey of the observations on the Martian bow shock and the upstream phenomena in the light of results from all the missions to date. It also discusses the kinetic properties of the Martian bow shock compared to the predictions of simulations studies. Then it examines the current status of understanding of these phenomena, including the possible sources of upstream low-frequency waves and the interpretations of localized disturbances in the upstream solar wind around Mars. Finally, it briefly discusses the open issues and questions that require further study.
\end{abstract}

\section{Introduction}

The study of planetary bow shocks and their upstream region provides important insight both into the behavior of astrophysical collisionless shocks and into the nature of the planetary obstacle to the solar wind flow. Since the flow velocity of 
the solar wind at each of the planets exceeds the velocity of compressional waves in the solar wind plasma, i.e., exceeds the fast magnetosonic speed, a shock wave must form in front of each planet if it does not absorb the incoming flow. The bow shock serves to slow down the flowing solar wind from supersonic to subsonic and to heat and deflect it around the planetary obstacle. The Mach number (magnetosonic, as well as sonic and Alfvénic) indicates the amount of deceleration and heating that occurs at the shock (the strength of the shock). The size of the solar wind obstacle largely determines the size of the shock relative to the planet. However, the physical size of the shock is also relevant since it dictates length scales for physical processes operating near the shock as well as the amount of space the upstream solar wind has available to be slowed and deflected around the obstacle.

Previous investigations of planetary bow shocks have established that their position, shape and jump conditions are functions of the upstream flow parameters and the nature of the solar wind - planet interaction (e.g., Spreiter and Stahara, 1980; Slavin et al., 1983; Russell, 1985). The first observations of the Martian bow shock were taken by Mariner 4 (Smith et al., 1965), from which the basic parameters of the shock were established. Other missions to Mars, such as Mars 2 and 3 (1972), Mars 5 (1975) and Phobos 2 (1989), continued the investigations (see reviews by Gringauz, 1981; Slavin and Holzer, 1982; Russell, 1985; Vaisberg, 1992a, b; Zakharov, 1992). The Phobos-2 spacecraft in particular provided a wealth of information about the plasma environment at relatively high altitude $(>850 \mathrm{~km})$ above Mars.

However, the exact nature of the solar wind interaction was elusive due to the lack of low altitude plasma and magnetic field measurements (e.g., Axford, 1991). It was not clear whether Mars, as an obstacle to the solar wind, was Earth-like, i.e., if Mars possesses an appreciable internal magnetic field, or if it was Venus-like or comet-like. The measured and/or inferred shock parameters such as standoff distance, flare angles, and jump conditions, provided much room for speculation about the nature of the Martian obstacle.

All this has changed with the arrival of Mars Global Surveyor (MGS) at Mars, and its acquisition of close-in magnetic field data (Acuña et al., 1998). MGS has now conclusively established that Mars is lacking, at present, an active dynamo. Thus the solar wind interaction is primarily Venus/comet-like. MGS has also shown the existence of magnetic remnants in localized, near-surface regions around the planet. These remnants, while complicating some detailed considerations at lower altitude, do not appear to influence the global structure of Mars's bow shock and upstream regions.

In comparison to Venus, Mars is located at a greater heliocentric distance and is also much smaller. Thus the solar wind density and interplanetary magnetic field (IMF) strength are smaller at Mars than at Venus, and therefore the Mach numbers are different. Further, the small planet size coupled with the low IMF strength at Mars makes the gyroradius of solar wind protons comparable to the size of the shock. At Mars, therefore, kinetic effects are potentially important. Finally, the 
Martian exosphere extends beyond the bow shock due to the much weaker Martian gravity, with significantly higher fluxes than at Venus. This has major implications for the actual solar wind/Mars interaction process, and therefore for the shock characteristics and upstream dynamics.

Below we review the current status of understanding for the phenomena that occur at the Martian bow shock and in the interplanetary medium upstream from this shock. We also discuss their implications for the nature of the global Mars solar wind interaction processes. Section 2 is devoted to the observations on the Martian bow shock in the light of results from all the missions to date and to the simulation studies, including the discussion on the kinetic properties. In Section 3 we review the observations on upstream phenomena at Mars. Section 4 is devoted to the possible sources of low-frequency waves upstream from the bow shock. Section 5 discusses the interpretations of localized disturbances in the upstream solar wind around Mars. Finally, in Section 6, we briefly comment the open issues and questions that require further study.

\section{The Bow Shock}

\subsection{BOW SHOCK STRUCTURE}

The plasma and magnetic field measurements performed by the Phobos- 2 spacecraft have confirmed previous observations made on Mars-2, 3, 5 and Mariner4 that the bow shock exists near Mars (Riedler et al., 1989; Schwingenschuh et al., 1990). The main shock substructures, a foot of reflected protons, overshoot, electron and ion foreshock were also clearly identified (Barabash and Lundin, 1993; Delva and Dubinin, 1998; Skalsky et al., 1992, 1993; Tatrallyay et al., 1997; Trotignon et al., 1992).

Phobos 2 provided sufficient magnetic field and plasma data for performing the first statistical analysis of the structure of the Martian bow shock by Tatrallyay et al. (1997). They used 82 crossings from the circular orbits of Phobos 2 in the terminator region $\left(80^{\circ}<S Z A<110^{\circ}\right)$ to investigate the characteristics of the transition layer at the Martian bow shock. They were interested in the investigation of the shock overshoot magnitude and thickness. They computed the overshoot magnitude as $\left(B_{M}-B_{2}\right) / B_{2}$, where $B_{M}$ is the maximum field value in the overshoot and $B_{2}$ is the theoretical downstream value computed from Spreiter and Stahara's (1980) gasdynamic model, using measured upstream field values. They found that the overshoot amplitude scales with the fast magnetosonic Mach number $M_{f}$ as typical for a fast-mode type shock. The thickness of the overshoot was taken as the distance along the shock normal between the beginning of the ion foot and the minimum of the first undershoot. The thickness of the Martian shock overshoots was in most of the cases between 0.5 and $2.5 r_{L}$ ( $r_{L}$ denotes the proton gyroradius calculated from the upstream solar wind speed and magnetic 


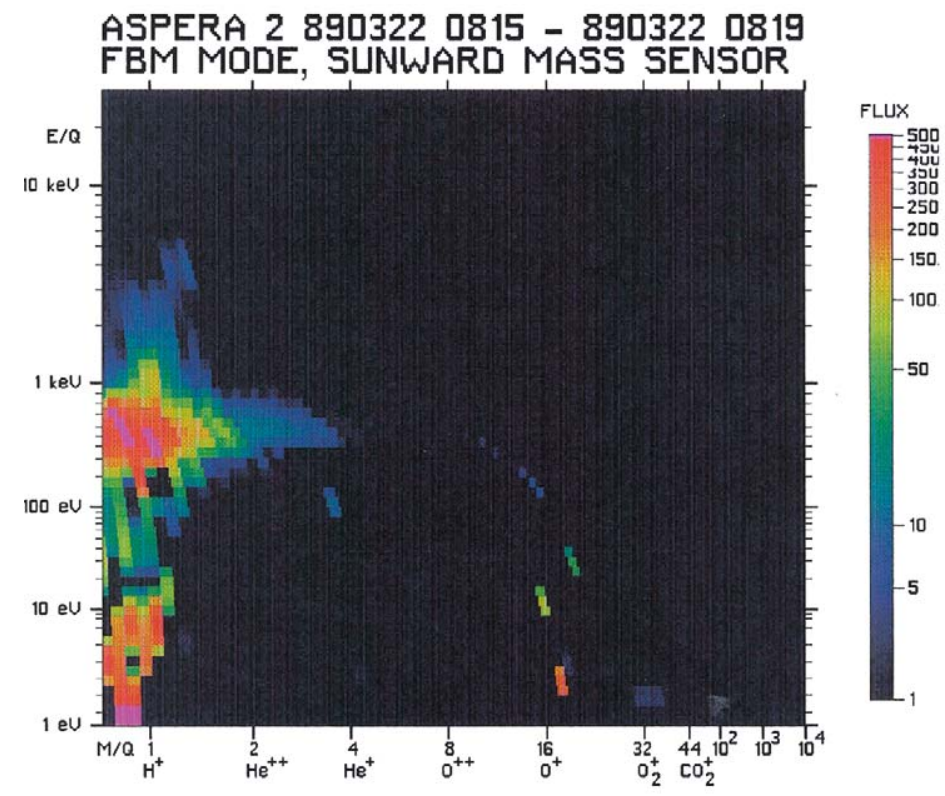

Figure 1. $E / q-m / q$ matrix collected by the ASPERA ion mass-spectrometer onboard Phobos-2 at the bow shock crossing near the terminator. Cold ions $\mathrm{H}^{+}, \mathrm{O}^{+}$(and traces of molecular ions with $m / q=32$ ) are of planetary origin.

field strength $\left.r_{L}=V_{1} / \omega_{c i 1}\right)$. In ion inertial length, the typical thickness was in the range $2<c / \omega_{p i}<8$.

On the other hand, some new features of the Martian bow shock were found. Dubinin et al. (1993) observed a sudden appearance of the low-energetic protons (and sometimes oxygen ions) at the bow shock. The energy of these ions was $\leq 10 \mathrm{eV} \mathrm{q}^{-1}$. Figure 1 shows the $E / q-M / q$ matrix measured by the ASPERA instrument during the $4 \mathrm{~min}$ interval corresponding to the crossing of the terminator bow shock. Three main proton populations are observed, shocked solar wind protons, more energetic ions that probably come from the reflected and subsequently accelerated protons and the protons with low energy. Spikes of the low-energy oxygen ions are also seen. Dubinin et al. (1993) attributed these low-energy particles to the ions originated from the extended hydrogen exosphere and hot oxygen corona. The authors confirmed the unique feature of the Martian bow shock associated with a small size of the obstacle to the solar wind. Moses et al. (1988) noted that the subsolar distance between the bow shock and the obstacle is about of the proton gyroradius based on its kinetic energy and therefore there is not enough space for the ion thermalization. Indeed, Dubinin et al. (1993) observed that a thermalization of the solar wind and exospheric protons occupies not only the whole magnetosheath (whose width near the terminator is much larger than in the subsolar region) but extends to the so-called 'plasma mantle'. 
MARINER IV, MARS 2, 3, \& 5 AND

PHOBOS-2 BOW SHOCK CROSSINGS

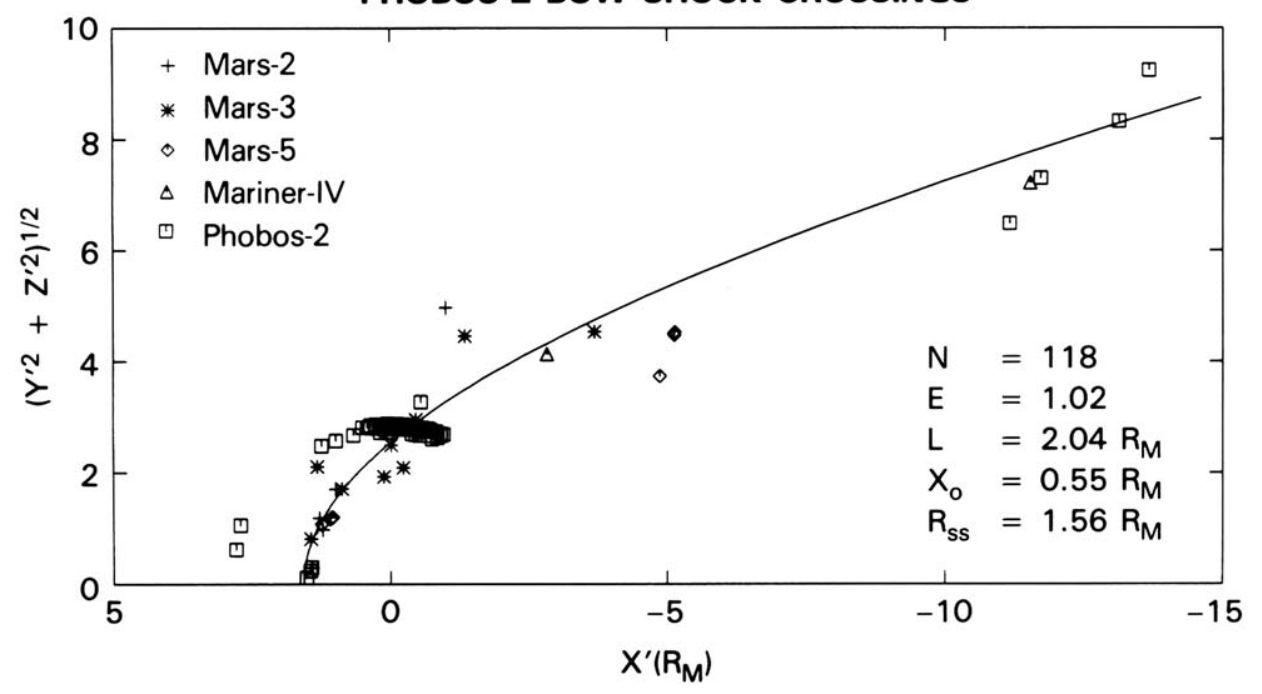

Figure 2. (Position and shape of the Mars bow shock based upon boundary encounters by Mariner IV, Mars 2, 3, and 5 and Phobos 2 along with a least-square, axisymmetric, second order fit in aberrated Mars Solar Orbital (MSO) coordinates (Slavin et al., 1991).

Since the number density of the exospheric ions was, at least, comparable with the number density of the shocked solar wind protons, their influence on the ion dynamics at the bow shock and magnetosheath can be important. The authors raised also an interesting question how to explain the high fluxes of planetary ions at such large distances from the planet $\left(R \sim 2.8 R_{M}\right)$.

The problem of unusual properties of the Martian bow shock was also addressed by Brecht (1997) in hybrid simulations of the solar wind-Mars interaction. Brecht (1997) argued that proton dissipation caused by ion reflection does not exist around Mars and concluded the difference of the "kinetic wave-like behavior of the Martian bow shock and standard hydrodynamic paradigm'. However, Skalsky et al. (1998) in their analysis of two typical bow shock crossings by Phobos-2 found that the observed changes in the bulk parameters are comparable with values expected for the MHD shocks and mechanisms responsible for the energy dissipation are similar to those operating at the Earth's bow shock. Further detailed measurements are necessary to resolve these discrepancies.

\subsection{THE AVERAGE LOCATION}

The particles and fields measurements collected by the various missions have clearly established that Mars possesses a fast mode bow shock whose structure closely resembles that of the other planets (e.g., Russell, 1985). The locations of the bow shock crossings returned by the pre-MGS missions (i.e., 2 from Mariner 4, 22 from 


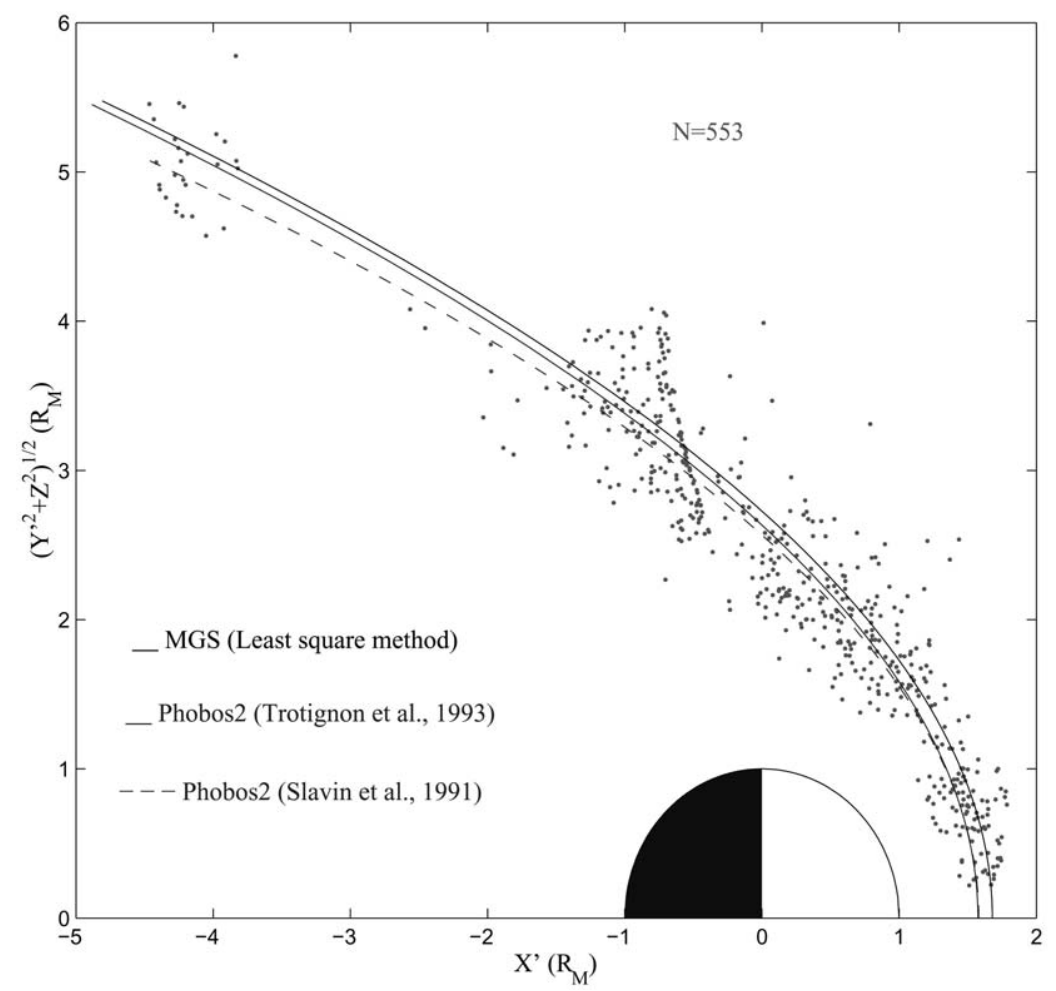

Figure 3. Position and shape of the Mars bow shock based upon boundary encounter by Mars Global Surveyor along with an axisymmetric second order fit in aberrated MSO coordinates (Vignes et al., 2000) compared to previous fits from Phobos 2 data (Slavin et al., 1991; Trotignon et al., 1993).

Mars 2, 3, and 5, and 94 from Phobos 2) are displayed in Figure 2 along with a solar wind aberrated, axisymmetric, second order least-square fit (Slavin et al., 1981, 1991). As shown, they span the region from the subsolar point to nearly 15 Mars radii downstream. These shock encounters nearly all occurred at low to moderate latitudes. Slavin et al. (1991) derived a planetocentric distance to the nose of the shock of $1.56 R_{M}$ and a Mach cone half-angle of $11.4 \mathrm{deg}$. This Mach cone implies an average magnetosonic Mach number of 5.1 which is close to the expected mean value of $\sim 6$ at Mars (Slavin and Holzer, 1982). Independent models of the bow shock by the different Phobos 2 instrument teams (Schwingenschuh et al., 1990; Verigin et al., 1991; Trotignon et al., 1991b) have produced very similar shock surfaces with an average subsolar shock distance $\sim 1.5 R_{M}$.

During the first year of the MGS mission, 553 shock crossings were identified from a total of 363 orbits and the mean shape and location of the shock determined (Vignes et al., 2000) using data from the magnetometer and electron reflectometer (MAG/ER). The MAG/ER package consisted of two magnetometers, which measure local magnetic field, and an electron reflectometer, which measures electrons fluxes in the energy range between $10 \mathrm{eV}-20 \mathrm{keV}$ (Acuña et al., 1992). In Figure 3 
a solar wind aberrated, axisymmetric fit of all these bow shock crossings using a conic section is displayed and compared with the earlier models of Slavin et al. (1991) and Trotignon et al. (1993). Comparing Figure 2 and Figure 3, MGS had many crossings on the dayside, while Phobos- 2 and the other missions provided a small but complementary number of crossings down the tail at greater distance than $10 R_{M}$. Overall, the agreement between the three surfaces based upon the MGS data alone, Phobos 2 alone, and the aggregate of the pre-MGS missions is very good.

Phobos 2 also investigated the variation in Martian bow shock location upon upstream parameters. The location of the shock was found to depend very weakly on the solar wind ram pressure $\sim\left(\rho V^{2}\right)^{0.02}$ (Verigin et al., 1993). This implies that the height of the subsolar Martian obstacle is highly stable. It was also found that the Martian bow shock moves from the planet as the sonic and Alfvénic Mach numbers decrease, in a manner usual for other planetary shocks (Verigin et al., 1997, 1999).

\subsection{SOLAR CYCLE VARIATIONS}

The position of the Mars bow shock is highly variable on a day-to-day basis, but the comparison between surfaces taken at different points in the solar cycle (see Figure 3) does not show anything like the $\sim 35 \%$ increase in the planetocentric distance to the shock in the terminator plane from solar minimum to maximum which was observed at Venus (Slavin et al., 1979). MGS and Phobos 2 observations give nearly the same mean bow shock fits for sunspot numbers of $\sim 30-90$ and $\sim 140-180$, respectively, suggesting that the mean surface is independent of the phase of the solar cycle (Vignes et al., 2000). In fact, the solar minimum shock surface from MGS is located at a slightly greater altitude than the solar maximum results from Phobos. However, this may be due to the high inclination of the MGS orbit as opposed to the very low inclination Phobos mission. It is known from studies of the bow shock at the Earth and other planets that the higher magnetosonic wave speed perpendicular, as opposed to along the local magnetic field, creates a small asymmetry in the cross section of the shock in the terminator plane with it being located at slightly greater altitudes over the poles as compared with the equator (e.g., Russell, 1985).

Slavin et al. $(1979,1983)$ proposed that the underlying cause of the solar cycle variation at Venus was the enhanced absorption of the incident solar wind via charge exchange at solar minimum as the ionopause recedes to lower altitudes and exposes more of the neutral atmosphere to the solar wind in the magnetosheath (Cravens et al., 1997). Conversely, Alexander and Russell (1985) and Russell et al. (1988) argued that the variation in bow shock location at Venus was due to the shock moving outward at solar maximum, because of enhanced pre-shock mass loading caused by the enhanced solar EUV flux. Zhang et al. (1990) found evidence in support of both mechanisms - subsolar absorption at solar minimum and 
enhanced mass loading at maximum. Finally, solar wind deceleration upstream of the Martian bow shock was detected by Phobos 2 orbiter and has been at least partially attributed to the effect of mass-loading operating at this planet (Verigin et al., 1991; Kotova et al., 1997). However, Dubinin et al. (2000a) proposed another interpretation from a multi-instrument data analysis of the observations in the upstream region which is detailed in Section 3.1. They showed that transient upstream wave phenomena interfere mass-loading signatures and can dominate over them.

\subsection{EFFECTS OF PICKUP IONS}

In order to study the variability of the BS position, Vignes et al. (2002) extrapolate each crossing to the terminator plane. Conic section fits were used to project them to the terminator plane using the same eccentricity and focus location than for the mean fit. This is helpful to examine the asymmetric dependence of the BS position relatively to the direction of locally upward electric field of convection. This dependence is related to the mass loading of the sheath by picked-up oxygen: the additional mass added to the flow in the North slows more the flow and creates a bigger effective obstacle there.

In the case of the solar wind interaction with Venus, mass loading of the solar wind results when atomics oxygen, the most important neutral species in the exosphere, are ionized. Atomics hydrogen, also present in Venus exosphere, are pickedup. Mass loading is thought to increase the distance of the bow shock from the planet and also to enhance the magnetic flux contained in the induced magnetotail.

In the case of Venus, the magnetic field direction was found to control the shock location. The pickup effect is at its maximum value when the solar wind flow and the IMF are perpendicular. This particular angle, the angle between the interplanetary magnetic field and the direction of the solar wind, is called the cone angle. The Venus BS was found to be sensitive to the cone angle (Alexander et al., 1986). Thus, to show the effect of the pick-up ions, we also need to look on the hemisphere of locally upward electric field of convection $(\mathbf{E}=-\mathbf{v} \times \mathbf{B})$. The relative clock angle correspond to the angle between the projection of the radial vector at the shock crossing location in the terminator plane and the local direction of the magnetic field also projected in this plane. Then, bow shock crossing in the northern hemisphere, by respect to the direction of the interplanetary magnetic field, occurs for clock angle between $0^{\circ}$ and $180^{\circ}$.

As the solar wind interactions with Mars and Venus are thought to be similar Vignes et al. (2002) look for evidence of bow shock asymmetry related to pickup ions. The MGS observations obtained during periods when the solar panels are illuminated are estimated to be accurate to $\sim 1 \mathrm{nT}$ (Acuña et al., 2001). As the value of the interplanetary magnetic field at Mars is typically a few nanotesla, this study of the mass loading effect on the Martian bow shock is made exclusively with cases where the IMF amplitude is strong enough to have an uncertainty on the direction of the IMF that is less than $20 \mathrm{deg}$, assuming this $\sim 1 \mathrm{nT}$ error. This data 


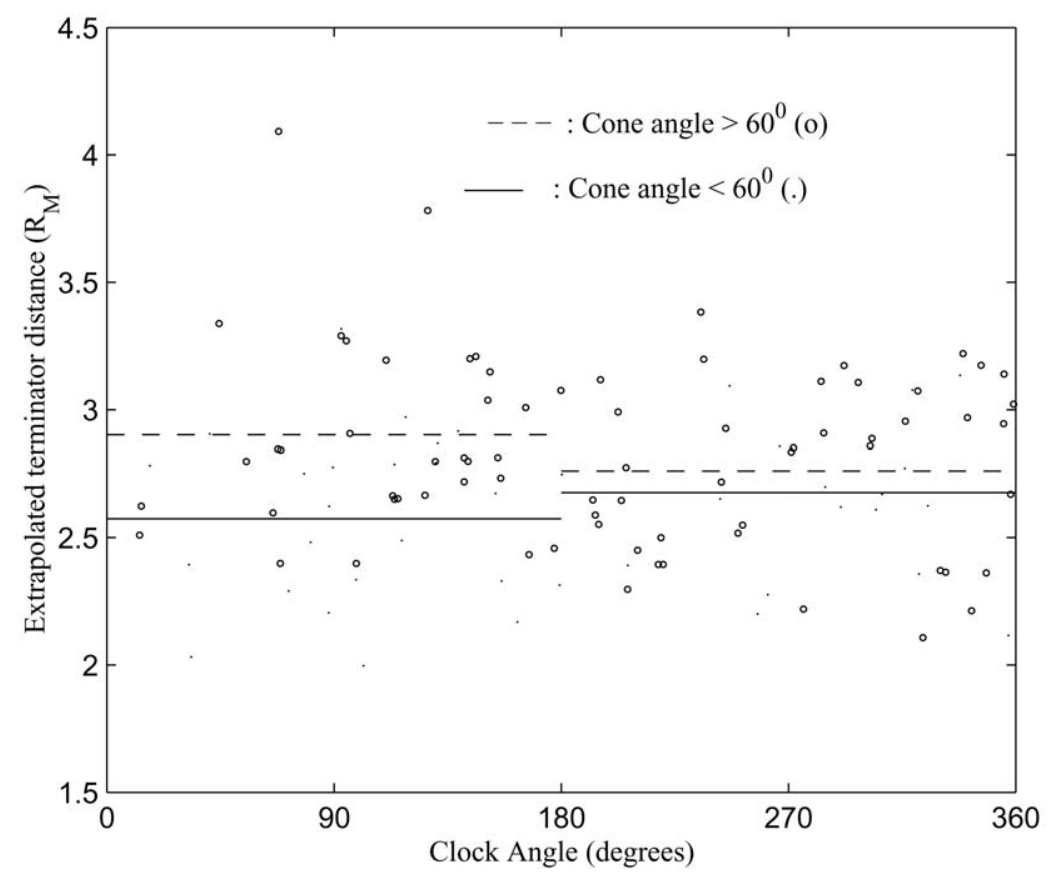

Figure 4. Location of Mars BS extrapolated to the terminator plane versus Clock Angle. Large and small cone angle are distinguished. Medians of the shock position are given depending on the hemisphere and depending on large or small cone angle (from Vignes et al., 2002).

restriction enables this study to look at cases of favorable pick-up effect: strong IMF, large cone angle and north clock angle.

Figure 4 shows the extrapolated terminator distance of Mars bow shock crossings as function of the clock angle. Vignes et al. (2002) distinguish cases of large and small cone angle in order to show the cumulative effect of large cone angle in the hemisphere of locally upward electric field. In the northern hemisphere, corresponding to the hemisphere of locally upward electric field, the mean value of the terminator distance for large cone angle $\left(2.9 R_{M}\right)$ is $13 \%$ greater than for small cone angle in the same hemisphere. While, in the southern hemisphere we found a small difference (3\%) between cases of large and small cone angle. Thus, the shock appears significantly farther from the planet in the hemisphere of locally upward electric field when the angle between the solar wind direction and the interplanetary magnetic field is large. This North-South asymmetry is consistent with the idea of asymmetric mass loading of the sheath by picked-up oxygen (Vignes et al., 2002). The additional mass added in the northern hemisphere slows more the flow and creates a bigger obstacle. Thus mass loading appears as playing some role on controlling the BS location. This role is expected to be more important during solar maximum since EUV fluxes increase and thus photo-ionization. 


\subsection{INFLUENCE OF CRUSTAL SOURCES}

During the Phobos 2 circular orbit phase, it was found that the magnetic field measurements contain spectral peaks at 12 and 24 hours. This periodicity was attributed the perturbation of the magnetic tail by a planetary magnetic field as Mars rotates about its spin axis (Mohlmann et al., 1991). MGS observations made from many periapsis passes over the northern hemisphere and at different longitudes only indicated the existence of weak fields $(\leq 5 \mathrm{nT})$ at low altitudes (down to $\sim 100 \mathrm{~km}$ ). This leads to an upper limit on a 'planet centered' intrinsic magnetic moment of $\sim 2 \times 10^{17} \mathrm{~A} \mathrm{~m}^{2}$ (or $2 \times 10^{20} \mathrm{G} \mathrm{cm}^{3}$ ) for the magnitude of a putative present-day Mars dipole equivalent to $0.5 \mathrm{nT}$ equatorial field (Acuña et al., 2001). However, MGS has found that while Mars has little or no low order intrinsic magnetic field, there are strong localized crustal magnetic fields mainly in the southern hemisphere (Acuña et al., 1999). The most intense magnetic crustal sources detected by MGSMAG/ER lie in the Terra Sirenum region $\left(150^{\circ} \mathrm{E}\right.$ to $240^{\circ} \mathrm{E} ; 30^{\circ} \mathrm{S}$ to $\left.85^{\circ} \mathrm{S}\right)$. Connerney et al. (1999) present analytical models of the sources in this region.

The total equivalent surface magnetic moment of this region, $\sim 1.3 \times 10^{17} \mathrm{~A} \mathrm{~m}^{2}$ (or $1.3 \times 10^{20} \mathrm{G} \mathrm{cm}^{3}$ ), is sufficiently high to shield portions of Mars from the solar wind. Using the usual pressure balance expressions (e.g., see Slavin and Holzer, 1982) the Terra Sirenum magnetic fields appear sufficient to stand-off the typical solar wind ram pressure at $\sim 1000 \mathrm{~km}$. However, in reality, some of the Terra Sirenum magnetic fields will close at lower altitudes via fields emanating from other anomalies. Furthermore, the net effect of the normal stress exerted by solar wind and the tangential stress due to reconnection between the interplanetary magnetic field and the Terra Sirenum magnetic fields will be to limit the altitude reached by these crustal fields and to spread them over a broader range of aerographic latitude and longitude (Brain et al., 2003). Theoretical model calculations (Ma et al., 2002), discussed in Section 6, have provided some good insight into how the crustal fields impact the interaction processes and create mini-magnetocylinders (Mitchell et al., 2001).

Figure 5 shows the extrapolated MGS terminator bow shock positions versus the longitude of the subsolar point at the time of the crossing in order to study the bow shock location dependence on crustal magnetic sources. If the variability of the bow shock position at all latitudes and longitudes were caused by high field anomalies embedded in the crust rotating with Mars, then a distinct maximum would be present over the Terra Sirenum region. However, the mean position of the shock averaged over all latitudes appears to be independent of the longitude of the subsolar point. This result is consistent with the Phobos analyses by Schwingenschuh et al. (1990) and Slavin et al. (1991) who also have found that the distance to the bow shock is independent of subsolar longitude in the Phobos 2 observations.

However, the high inclination of the MGS orbit relative to Phobos 2 also provides an opportunity to examine bow shock location as a function of latitude. This is significant, because the vast majority of the strong magnetic anomalies detected 


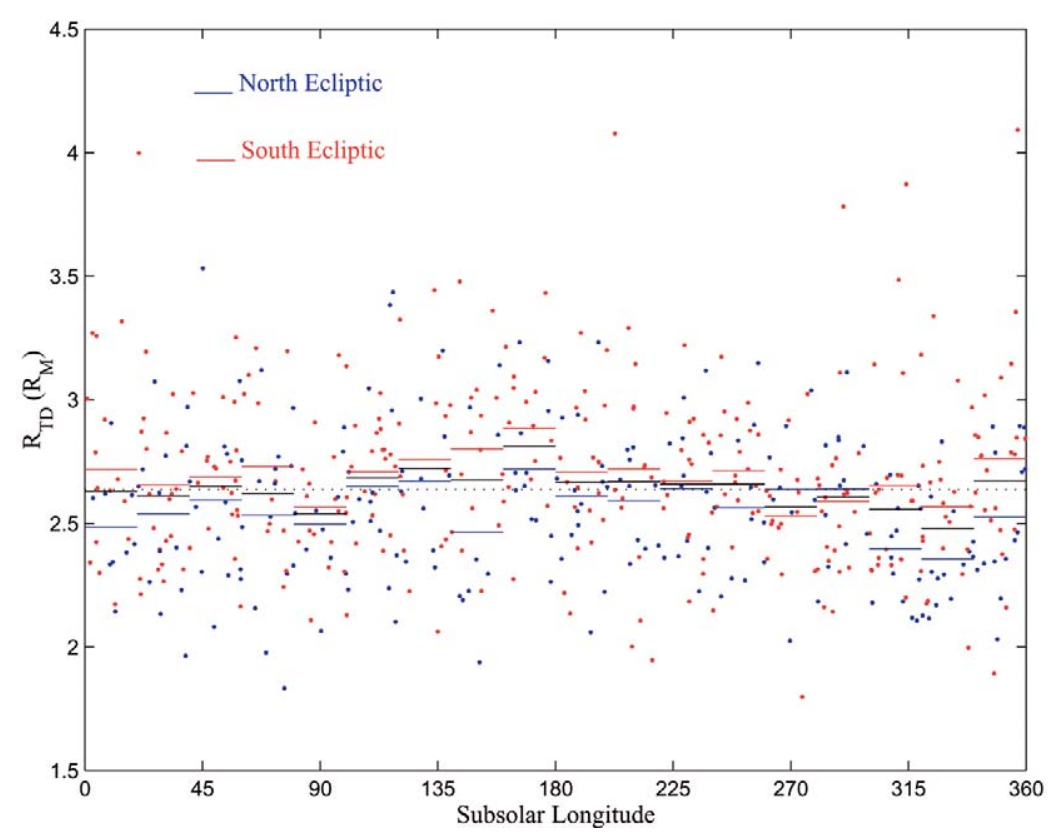

Figure 5. MGS bow shock crossings mapped into the terminator plane and plotted against the longitude of the subsolar point at the time of each event. Crossings over the northern and southern hemispheres are plotted in blue and red, respectively, and medians are indicated as horizontal line segments.

by MGS are located south of the Martian equator. Figure 5 plots the shock crossings over the northern and southern hemispheres in blue and red, respectively, and indicates the corresponding medians as horizontal line segments. The results do show a clear tendency for the Martian bow shock to be found at slightly greater heights over the southern hemisphere, i.e., the red median bars are higher than the blue medians in 16 of 18 longitude sectors. If verified by further analysis of the MGS measurements, the asymmetric distribution of bow shock crossings suggests that the effect of the crustal magnetic anomalies on the solar wind flow about Mars are felt most strongly above and just downstream of the southern hemisphere.

\subsection{BOW SHOCK SIMULATIONS AND RELATED PROBLEMS}

Simulations should help to find out what are the reasons for the different signatures seen in the observations of the Martian bow shock. The fact that the bow shock at Mars is located close to the planet that there is not enough space for the solar wind protons to fulfill complete gyro motions was the motivation for kinetic studies using hybrid simulations (Moore et al., 1991; Brecht, 1990, 1997; Brecht et al., 1993). Another point of interest is the different variability of the bow shock location at Venus and Mars with the solar cycle. Models should give an answer why it happens. At Mars a specific situation arises from the existence of an extended 


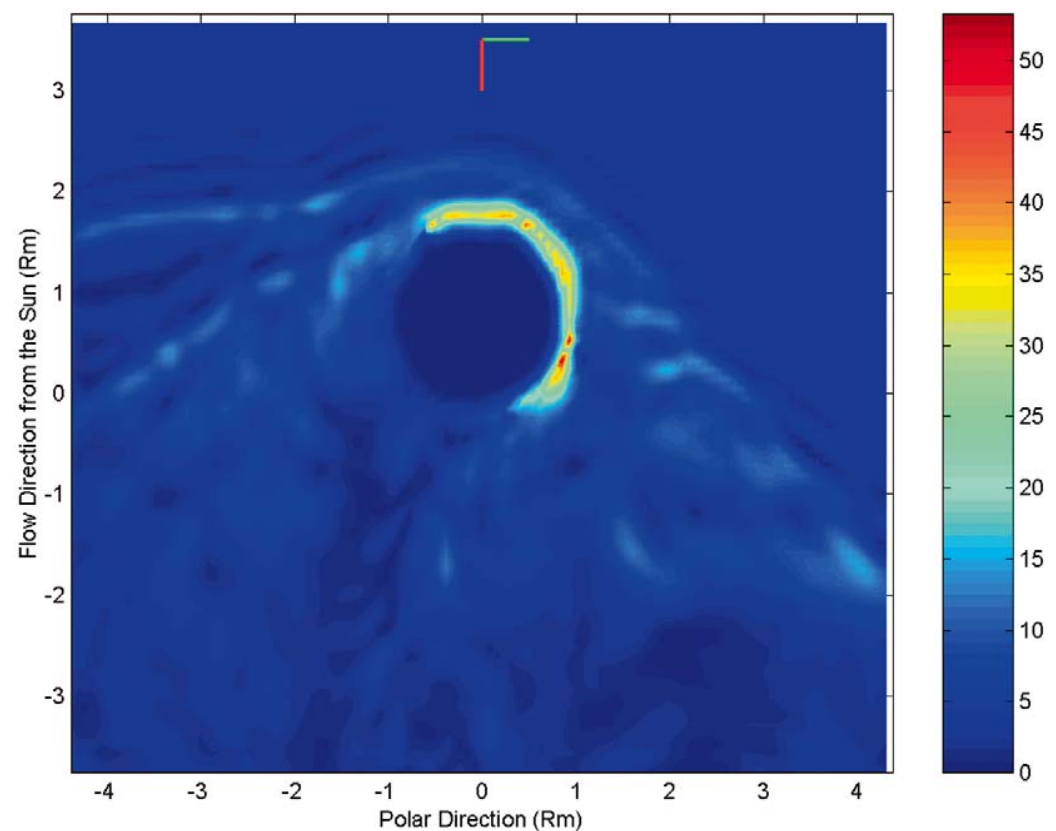

Figure 6. This figure shows the tendency for the magnetic field to convect over the Northern magnetic Pole defined by the direction of the convection electric field $-\mathbf{V} \times \mathbf{B}$. One can see the tendency for the field in the barrier region to coalesce. Further the asymmetry of the shock and barrier structure over the poles is seen (Brecht, 2002).

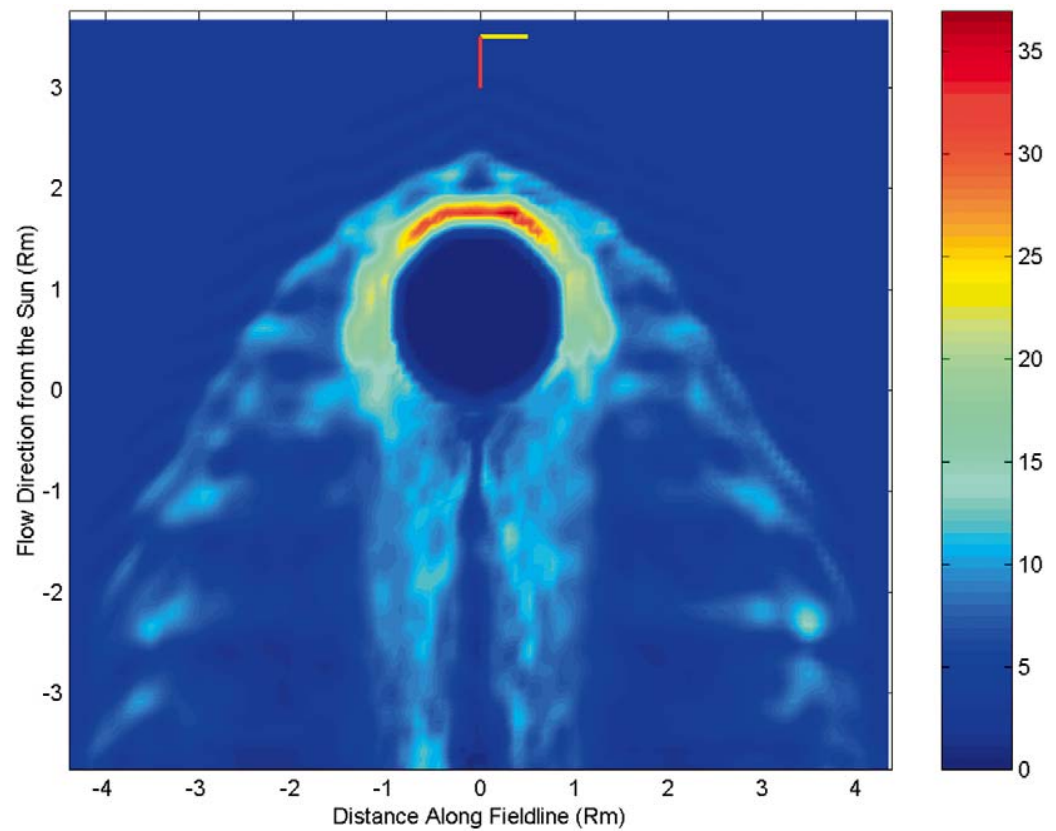

Figure 7. This figure shows the same simulation but the opposite plane. In this plane the magnetic field and the solar wind exist. This is nominally the ecliptic plane being plotted. The extreme symmetry is remarkable since this simulation is fully kinetic and one is seeing only a slice through the three dimensional simulation (Brecht, 2002). 
hydrogen/oxygen exosphere which may already lead to the interaction of the solar wind with planetary ions in the vicinity of the bow shock. The question is how this process modifies the shock structure. Finally one has to solve the problem about what are the dominant processes in shock formation. After the MGS mission there are no doubts that the shock at Mars is caused by braking of the solar wind due to its interaction with the exospheric/ionospheric plasma which acts as an obstacle. But, the nature of the 'obstacle boundary' is still a subject of ongoing discussions which concern the interpretation of the so-called 'magnetic pile-up boundary' (MPB) seen in MGS data; see the companion review paper by Nagy et al. (this issue). In this context one can also hope to find a final answer what processes determine the shape of the bow shock, which means to clarify whether it is symmetric or asymmetric with respect to the direction of the interplanetary magnetic field and the convection electric field. From different models different answers have been obtained.

In first three-dimensional kinetic simulations (kinetic ions and mass-less fluid electrons) of the bow shock of Venus by Moore et al. (1991), and Mars by Brecht $(1990,1997)$ and Brecht et al. (1993) the planet was considered as a obstacle with an absorbing boundary. The aim of these studies was to find out the effect of finite Larmor radius on the structure of the bow shock. They showed that the large Larmor radius of the solar wind protons prevents the formation of a traditional collisionless shock. An asymmetry was found in the direction of the convection electric field as shown in Figure 6 (Brecht, 2002). One interesting note is that the asymmetry is a function of the Parker spiral angle, because the angle determines the relative strength of the solar wind convection electric field. In the ecliptic plane both the data and kinetic simulations show a very symmetric result, as shown in Figure 7 (Brecht, 2002). In simulations by Shimazu (1999) using a reflecting boundary similar results have been obtained. The observed asymmetry of the magnetic field configuration is interpreted as result of differences in the ion acceleration due to the convection electric field.

Another feature of the Martian bow shock as revealed by kinetic simulation is that because of the closeness between the bow shock and the planet, there is barely room for the number of gyroradii necessary for thermalization. Indeed, while simulations of Venus show that the plasma is thermalized when passing through the shock, the simulations of Mars show that the plasma is not completely thermalized (Brecht et al., 1993). This is coupled with the strong wave activity generated by the high Alfvénic Mach number shock created at Mars. For the normal solar wind parameters, the Alfvén Mach number is above 10. A collisionless shock with a Alfvén Mach number of this size or greater is very electromagnetically active and will generate a substantial suite of electromagnetic waves with frequencies ranging from the whistler down to the electromagnetic modes with frequencies near or even below the ion gyrofrequency, $T_{c i}$ (Thomas and Brecht, 1987)

Shimazu (2001) has modeled more realistic conditions with respect to the situation at Mars by treating the planet as an ionized gaseous body. In this model planetary ions are distributed uniformly in a sphere of a radius $R=D / 8$ which 

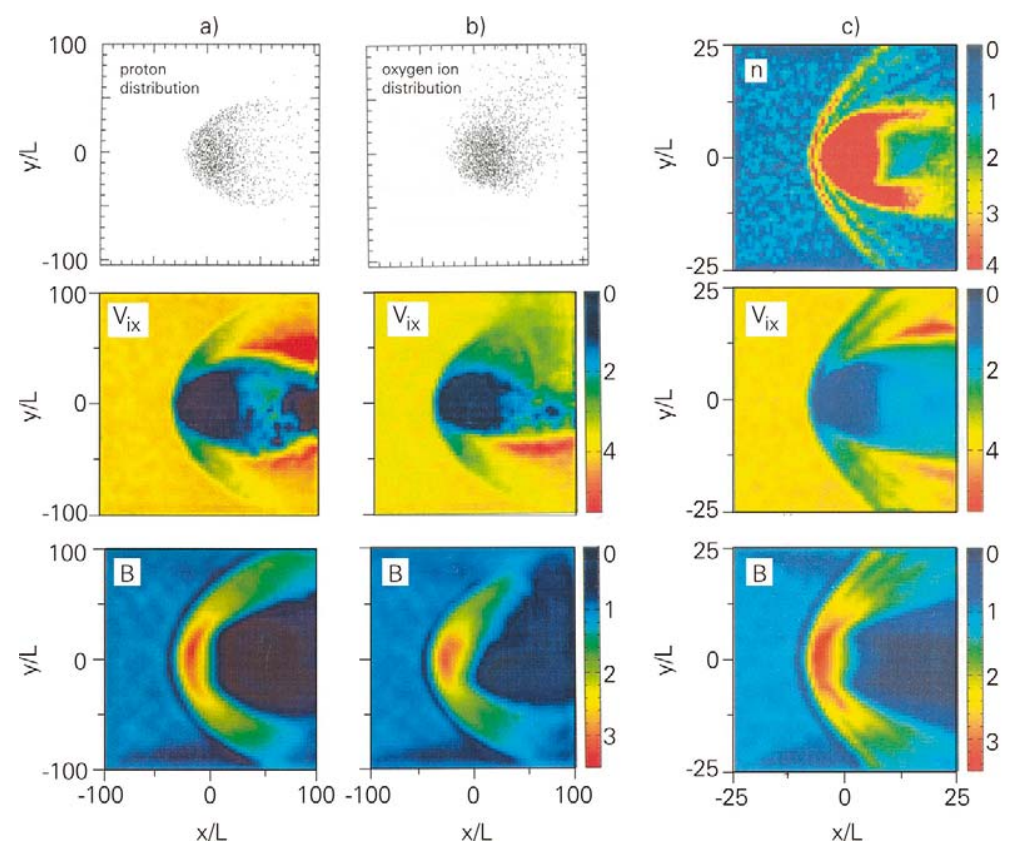

Figure 8. 3D hybrid code simulations of solar wind - Mars interaction, after Shimazu (2001). The ionosphere of Mars is modeled by a source of planetary ions, distributed uniformly in a sphere of radius $R=d / 8$ ( $d$ : box size); (a) proton source, $d=200 L$ ( $L$ : proton skin length); (b) source of oxygen ions, the same parameters as in (a); (c) proton source, $d=50 L$. The Mach number is $M_{\mathrm{A}}=4$.
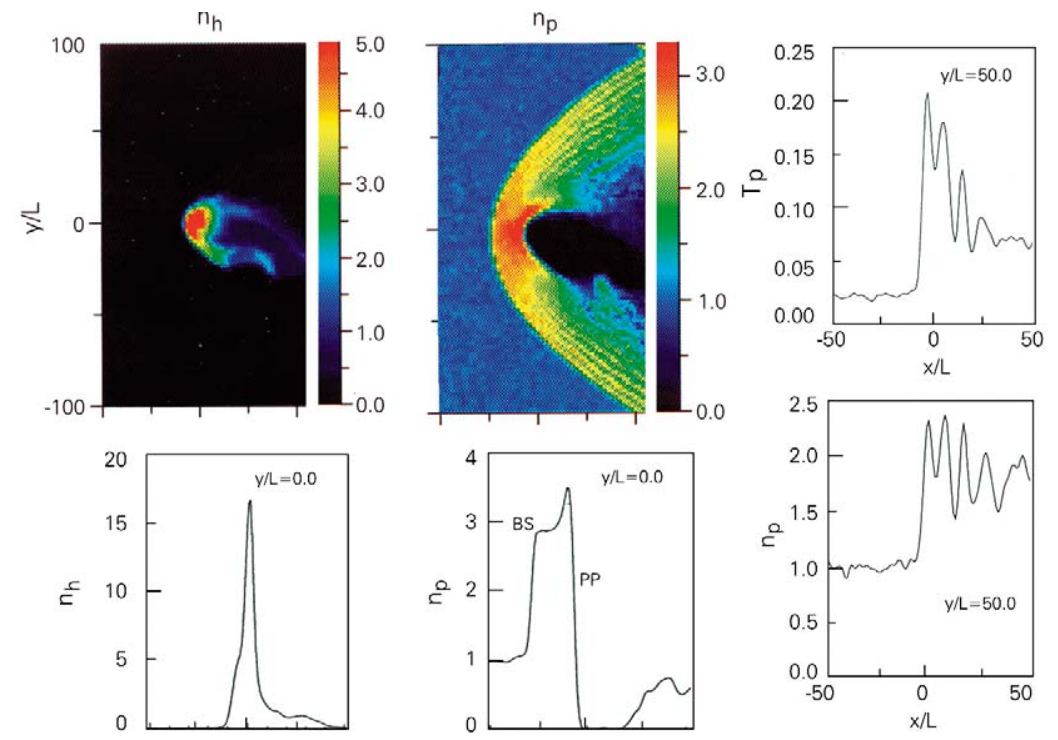

Figure 9. 2D hybrid code simulations of solar wind - (cometary) exosphere interaction, after Sauer and Dubinin (2003). The color plots on top show the heavy ion density $n_{h}$ (left) and the proton density $n_{p}$ (right). The most remarkable signature is the formation of a proton cavity. The cuts along $x$ trough the center of the box (middle two panels) clearly show that the proton density jumps down to about zero where the heavy ion density strongly increases (change of ion composition). The lower panels show that the bow shock is structured; $T_{p}$ is the proton temperature. 
is placed at the center of the simulation box with the size $D$. The supply rate $q$ of ionospheric ions was assumed to be uniform and constant, and with a rate $q=0.256 \omega_{p}\left(\omega_{p}\right.$ : proton plasma frequency) the supply and escape to the tail were almost in balance. This rate corresponds to an ion production rate of $\approx 3 \times 10^{24} \mathrm{~s}^{-1}$ which is about 5 times smaller than the estimated loss rate of Mars from Phobos-2 measurements.

Results of the simulations are summarized in Figure 8. The solar wind Mach number is $M_{A}=4.0$. Three cases are shown. In panel a) and b) the radius of the sphere is $R=6.4 \lambda$ where $\lambda=M_{A} L$ ( $L=$ proton skin length) using protons (a) and oxygen ions (b) as planetary ions. Panel c) shows results for a small planet $(R=1.6 \lambda)$ with proton production. The magnetic field is directed out of the plane ( $z$ axis), that means, the convection electric field $E$ shows in positive $y$-direction.

For oxygen ion production (Figure 8(b)) a clear asymmetry in the direction of $E$ can be seen. This is caused by the oxygen ions escaping from the side of the planet to which the electric field is pointing. The calculated asymmetry in the magnetic barrier intensity is consistent with the observations. The asymmetry in the shock size is the same as calculated by Brecht and Ferrante (1991) and Shimazu (1999), however, do not match the asymmetry observed near either Venus (Alexander $e t$ al., 1986; Russell et al., 1988) or Mars (Zhang et al., 1991). This might be an effect of mass loading far away from the planet which is not described by the present ionospheric model with the abrupt onset of planetary ion production. In the case of a reduced planet size a multiple-shock structure is observed in the simulations. This is clearly visible as ray structure in the upper panel of Figure 8(c) where the density distribution is shown. Although an explanation is given due to finite Larmor radius effects, one cannot exclude that a beam-plasma configuration by reflected protons might cause the periodic density structure. This conclusion is supported by the fact that similar phenomena also appear for larger 'obstacles', as shown later.

A further interesting aspect of the Shimazu (2001) paper concerns the discussion about the behavior of the solar wind if it interacts with the plasma of planetary origin, even if this is not the main focus of this paper. It is stated that the two kinds of ions (solar wind and ionosphere) do not mix very much and that a reflecting or absorbing boundary as taken in several earlier models (Brecht, 1990, 1997; Moore et al., 1991; Brecht et al., 1993; Shimazu, 1999) appears to be an extreme and unrealistic assumption.

In a recent work by Sauer and Dubinin (2003) 2D hybrid code simulations which were originally done to study how the solar wind interacts with weakly outgassing comets (Lipatov et al., 1997) have been extended to cometary-type objects with larger production rates. The results shown in Figure 9 are relevant for the conditions at Mars where extended hydrogen and oxygen exospheres seem to have essential influence on the solar wind before it interacts with the denser ionosphere closer to the planet. Figure 9 shows results for a cometary-type source with a production rate of $Q=10^{27} \mathrm{~s}^{-1}$ (photo-ionization rate $10^{-6} \mathrm{~s}^{-1}$ ) for an incoming solar wind with $M_{A}=5.0$. The simulation box was $200 \times 200$ in units of 
the proton skin length $L$. The center of the source is in $x=y=0$. More details are given in Lipatov et al. (1997). The color plots on top of Figure 9 show the heavy ion distribution (left) and the solar wind density distribution (right). The shock is nearly symmetric, although the motion of the main heavy ion population opposite to the convection electric field direction causes an asymmetric picture. This motion results from the momentum conservation in $y$-direction: the momentum of the lowdense high-velocity ions escaping along the convection electric field is balanced by the momentum of the dense ions moving slowly in the opposite direction. Further, there are two remarkable effects to mention. One is the clear bow shock structuring with wave lengths of about $5 L$ (visible in the cuts shown in the lower panels of Figure 9) which are probably caused by steepened magneto-sonic waves as discussed in earlier papers (Hopcroft and Chapman, 2001; Lipatov et al., 2002) and in a recent publication by Bagdonat and Motschmann (2002).

The other important result is related to the crucial problem of 'obstacle' formation at solar wind interaction with a source of cometary/planetary ions, as it happens at comets, Mars and Venus. The color plots of the density distributions in Figure 9 and the cuts below clearly show that a boundary is formed where the solar wind proton density sharply decreases and the heavy ion density jumps up to values of about the (upstream) peak proton density. So, a 'replacement' of protons by heavy ions takes place. An accompanying effect (not shown here) is an abrupt pile up of the magnetic field. The same characteristic features of the 'obstacle boundary' have been described before with the help of two-ion fluid simulations (Sauer et al., 1994, 1995a; Sauer and Dubinin, 2000) demonstrating that the macroscopic momentum coupling and not single-particle effects are important in forming the boundary. The Phobos and MGS missions to Mars observed such new type of plasma boundary. It has been shown recently from the analysis of the magnetic field that this boundary is also present at Venus (Bertucci et al., 2003a,b) and coincides with the proposed 'mantle boundary' both at Venus and Mars (Vaisberg and Zelenyi, 1984; Zelenyi and Vaisberg, 1985). It is now called 'magnetic pile-up boundary' and will be described in more details in Nagy et al. (this issue).

Another class of simulations, not directly related to the Mars bow shock, which generally help to understand the pick-up dynamics near bodies with an ionized environment have been done using both hybrid codes (Lipatov et al., 1997; Hopcroft and Chapman, 2001; Bagdonat and Motschmann, 2002) and two-ion MHD models (Bogdanov et al., 1996; Sauer and Dubinin, 2000; Szegö et al., 2000). The aim of these studies was to see how the character of solar wind interaction with a source of cometary/planetary ions changes if the production rate varies from low to high values and what are the signatures of the related plasma structures. In all models, cited above, a neutral gas source of $n_{0}=Q /\left(4 \pi r^{2} v_{0}\right)$ was taken, where $Q$ is the production rate in molecules per second, $r$ the distant from the center of the source and $v_{0}$ the thermal velocity of the ejected neutrals. The difference to the conditions at planets is that, instead of the $1 / r^{2}$-profile for comets a source with 
exponential dependence $\left(\sim e^{-r / h}\right)$ must be taken. In this sense the following results give hints how the existence of planetary exospheres, which in some cases (as at Mars) extends far upstream the bow shock, may influence the global interaction region.

As shown first in a paper by Bogdanov et al. (1996), the Mach number and the production rate are essential parameters which determine the character of the interaction. If the Mach number is fixed at $M_{\mathrm{A}}=5$, for example, a transition from a very asymmetric type of interaction in form of Mach cones to nearly symmetric bow shock structures takes place if the production rate is enhanced from $Q \sim 10^{25} \mathrm{~s}^{-1}$ to $Q \sim 10^{27} \mathrm{~s}^{-1}$. First, in Figure 10(a), plasma structures are shown for a low production rate, comparing kinetic hybrid code results (Bagdonat and Motschmann, 2002) with the outcome of two-ion fluid MHD simulations (Sauer and Dubinin, 2000; Szegö et al., 2000). As a somewhat surprising fact the overall pattern, showing the heavy-ion cycloid in the convection electric field direction and the related strongly asymmetric Mach cone, coincides very well for both models. This demonstrates that the electromagnetic coupling of the proton and heavy-ion fluid dynamics, which is obviously well described in the two-ion fluid approach, primarily causes the observed plasma behavior. The reason of the strong asymmetry in the magnetic field is due to the proton deflection opposite to the cycloidal motion of the heavy ions by the Lorentz force which arises from the differential motion of high-speed protons and slowly moving heavies near the center of the source. So, even for relatively weak interaction processes test particle description and one-fluid models will fail.

With increasing production rate the interaction pattern becomes more symmetric. This is illustrated in Figure 10(b) where results of two-ion fluid simulations are shown for two production rates, $Q \approx 10^{26} \mathrm{~s}^{-1}$ (left panels) and $Q \approx 10^{27} \mathrm{~s}^{-1}$. For the lower production rate there is a clear asymmetry that arises from the strongly different motion of the two types of ions, as discussed before. If $Q$ further increases, the cycloidal motion is suppressed and the heavy ions begin to form raylike structures which is accompanied by a relatively symmetric magnetic field pattern which is confirmed by hybrid code simulations, e.g., Lipatov et al. (1997). More discussion about these effects and their relevance for the Martian bow shock one can find in papers by Dubinin et al. (1998), Sauer et al. (1998b) and Dubinin and Sauer (1999).

In summary one can state that progress was made over the last years in simulating the bow shock of Mars using different approaches and models. It seems that the existence of an extended exosphere is an essential signature at Mars, which makes it necessary to take into account the effect of pick-up processes on the bow shock structure. Up to now, this was insufficiently done and, hopefully, in next future better models for describing the particular conditions of the solar wind interaction with the exosphere/ionosphere of Mars will improve the present situation. 
a)
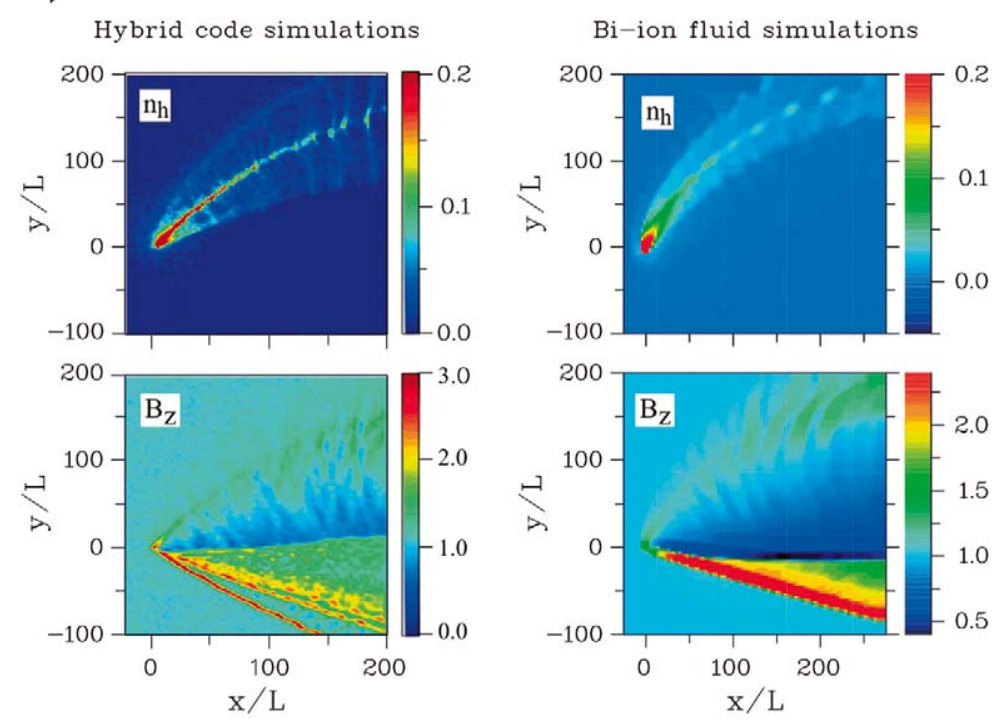

b)
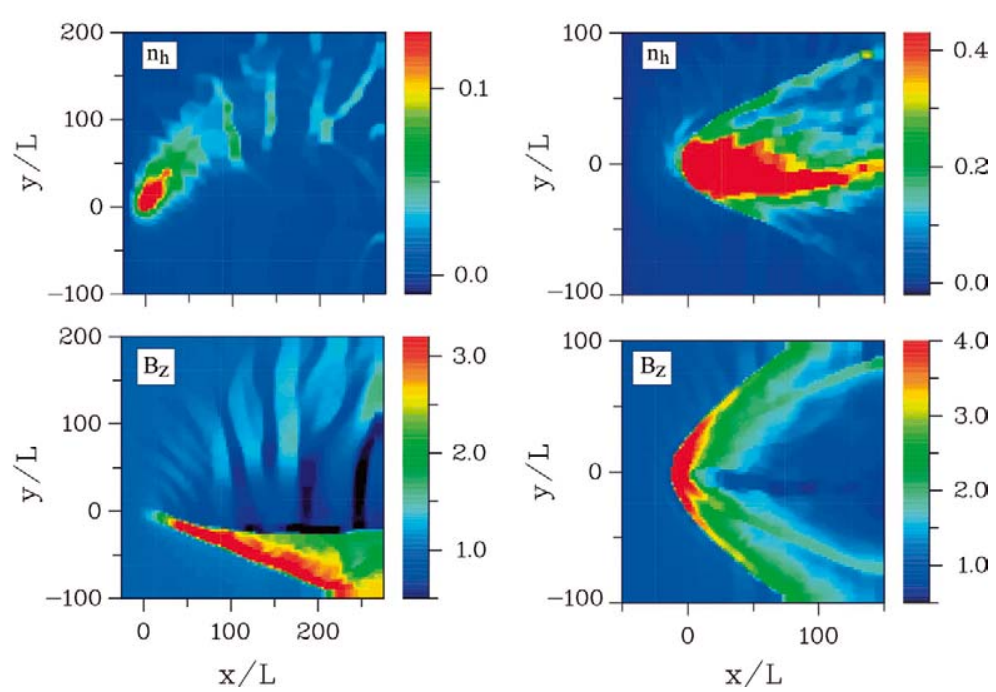

Figure 10. Influence of mass loading on the bow shock structure: Interaction pattern for different production rates. (a) Comparison between hybrid code results (left panels), after Bagdonat and Motschmann (2002) with bi-ion fluid simulations (right panels), after Sauer and Dubinin (1999); $M_{\mathrm{A}}=10, Q \approx 10^{26} \mathrm{~s}^{-1}$. The good agreement indicates the crucial role of momentum exchange between both ion fluids, which is obviously well described in the fluid approach. (b) Interaction pattern from bi-ion fluid simulations, after Sauer and Dubinin (1999), for two production rates: $Q \approx 10^{25} \mathrm{~s}^{-1}$ (left panels) and $Q \approx 10^{26} \mathrm{~s}^{-1}$ (right panels), $M_{\mathrm{A}}=5$. 


\section{Upstream Phenomena}

\subsection{MASS LOADING AND FORESHOCK PHENOMENA}

Due to the lack of the shielding global intrinsic magnetic field the solar wind exposes an extended exosphere/atmosphere of Mars far from the bow shock. Photoionization, electron impact and charge exchange lead to the creation of newly born ions which may affect the incoming solar wind. At large distances the most abundant neutral components are hydrogen atoms which could reach very high altitudes as the consequence of a weak gravitational attraction of Mars. Fast oxygen atoms originated in the process of the dissociative recombination of molecular oxygen $\mathrm{O}_{2}^{+}$which dominates at the ionospheric heights may additionally contribute to the neutral exosphere. If these neutral gaseous shells are not very rare mass-loading effects similar to be observed near the comets could be expected at Mars.

Indeed, already the first measurements made by the Phobos 2 spacecraft were interpreted as the evidence of strong mass loading. Figure 11 shows the variations in the potential difference between a conductive spacecraft at floating potential and the electric field probe, negatively biased in relation to the spacecraft along four successive elliptical orbits as a function of time centered around times of periapsis (Pedersen et al., 1991). A gradual decrease of the potential difference, which is approximately inversely proportional to the electron number density, indicates an increase in the plasma density with decreasing distance to the planet. Under some assumptions, Pedersen et al. (1991) derived the electron number density and found that a distinct increase in $n_{e}$ starts far upstream from the Martian bow shock. The electron number density increased by $1-2 \mathrm{~cm}^{-3}$ between the undisturbed solar wind and the outer limit of the shock foot.

Verigin et al. (1991) have reported the essential solar wind deceleration upstream the bow shock along the elliptical orbits and attributed these observations to mass loading on the hot oxygen corona. In front of the bow shock the decrement in the bulk speed reaches $\sim 100 \mathrm{~km} \mathrm{~s}^{-1}$. Assuming an instantaneous assimilation of planetary ions to the solar wind, Verigin et al. (1991) suggested that the oxygen corona near Mars is five times denser than that predicted by Ip (1988). Since the model by Ip (1988) overestimated the number density in the hot oxygen corona for solar maximum conditions at a factor of 2-3 (Ip, 1990) the value obtained by Verigin et al. (1991) far exceeds even the extreme number density of oxygen atoms. Later Kotova et al. (1997) have analyzed the TAUS observations on the circular orbits and concluded that deceleration of the solar wind occurs on oxygen corona with the number density at $\sim 3$ times higher than the values predicted by the extreme model. Recent reexamination of the altitude distribution of neutrals in the hot oxygen corona at Mars for maximum solar activity made by Kim et al. (1998) gives the oxygen density nearly one order of magnitude less than the values estimated by Kotova et al. (1997). There is also another serious problem in the mass-loading scenario. Unlike the comet case, there is not enough room near Mars 

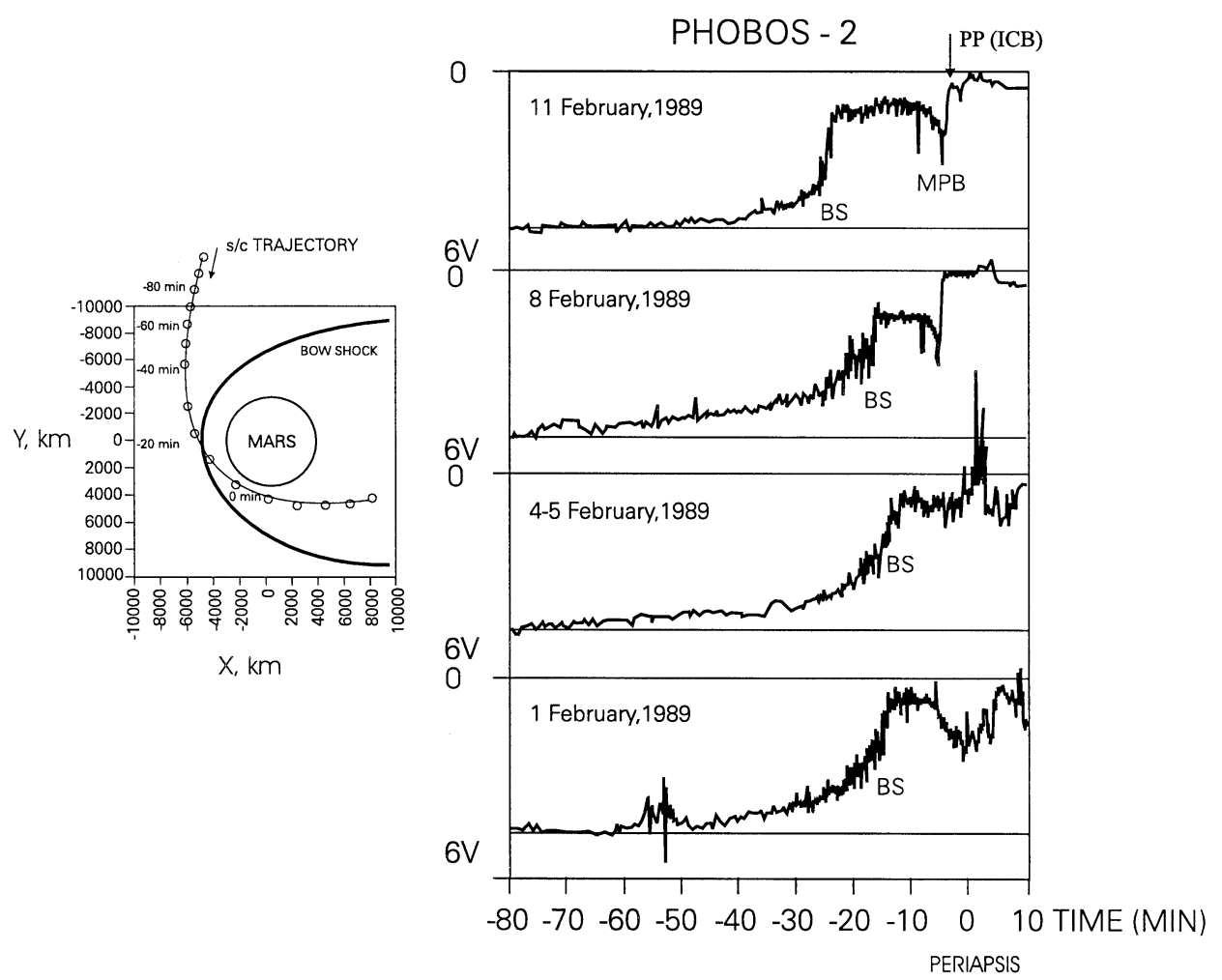

Figure 11. The potential difference between a conductive spacecraft at floating potential and the electric field probe, negatively biased in relations to the satellite body, is approximately inversely proportional to the electron number density. The data for the four successive elliptical orbits around Mars centered in time relative to the periapsis passage are presented (adapted from Pedersen et al., 1991). The orbit of Phobos-2 in XY ecliptic plane is shown too. When Phobos-2 was approaching Mars within the ion foreshock (1, 4 and 8 February 1989) the onset of increase in electron number density took place far from the planet. When the spacecraft was traveling outside the foreshock (11 February) signatures of increase in $\mathrm{Ne}$ were observed only after the crossing of the foreshock boundary close to the bow shock.

for assimilation of pickup ions. Gyroradius of pickup oxygen ions is much larger than the deceleration distance and the assumption of ion assimilation into the solar wind is not valid.

Dubinin et al. (1994) have shown that a decrease in the difference potential measured by the PWS instrument was observed only when the spacecraft was approaching Mars within the foreshock. On February 1, 4 and 8 Phobos 2 was moving in the foreshock only with short excursions into the solar wind. On February 11 the spacecraft was out the foreshock most of the time and crossed the foreshock boundary only downstream from the tangent point close to the bow shock. The foreshock geometry and the position of the spacecraft with respect to the foreshock were reliably determined by different methods (Dubinin et al., 2000a). It was suggested 
COMPARISON OF THE MODEL WITH OBSERVATIONS
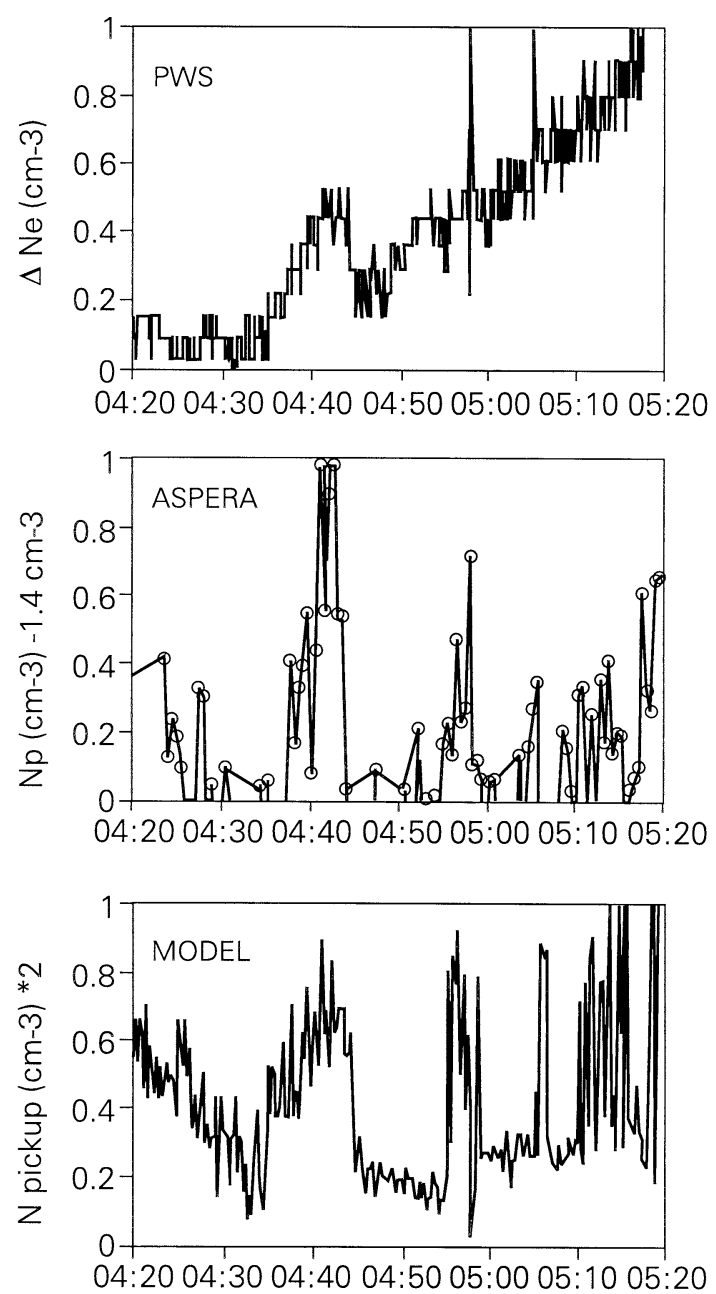

Figure 12. Comparison of observed and simulated variations in plasma density along the orbit of Phobos-2 on February 8, 1989. From top to the bottom are the variations in the electron number density derived from the measurements of the floating potential, variations in the proton number density measured by the ASPERA instrument, modeled values of the density variations (adapted from Dubinin et al., 1994).

that such asymmetry is caused by reflection at the bow shock of pickup $\mathrm{H}^{+}$ions originating from the extended atomic hydrogen atmosphere (Dubinin et al., 1994, 1995). The importance of pickup ion reflection is motivated by the significant fraction of pickup protons with small normal velocity components at the bow shock that prevent their overcoming through electrostatic potential barrier.

Dubinin et al. $(1994,1995)$ have simulated the dynamics of pickup protons and have shown that their altitude distribution becomes strongly asymmetrical due 
to reflection at the bow shock. The model qualitatively well explains not only the global asymmetry in the floating potential measurements but also a fine structure of the potential variations. Figure 12 compares changes in the total number density as compared to the unperturbed solar wind: $\Delta n_{e}$ derived from the difference potential measurements (the bottom panel), $\Delta n_{p}$ (ASPERA) (middle) and $\Delta n_{\text {pickup }}$ derived from the model (the top) for one of the elliptical orbits. A fraction of reflected ions is mass-dependent $\sim\left(m_{p} / m_{\text {pickup }}\right)^{1 / 2}$ where $m_{p}$ and $m_{\text {pickup }}$ are masses of protons and pickup ions, respectively and therefore heavy ion species $\left(\mathrm{He}^{+}, \mathrm{O}^{+}\right)$ less effectively participate in this process. It has been shown that the flux of backstreaming particles in the Martian foreshock may be anomalously high (>10\%) and cause a slowdown of the solar wind (Ip, 1992; Dubinin et al., 2000b). Note that the deceleration of the solar wind is also observed in the Earth's foreshock where the flux of backstreaming ions is much less (Bonifazi et al., 1980; Zhang et al., 1995, 1997). Particles reflected at the bow shock may turn back by the Lorentz force and execute several reflections. Strong magnetic and electric field turbulence observed near the bow shock (Grard et al., 1991) may significantly increase the number of ion oscillations and provide the formation of a peculiar trap for pickup protons that in their turn may lead to a strong solar wind deceleration in front of the bow shock observed by the TAUS instrument.

Multi-instrument data analysis of the observations in the upstream region made by Dubinin et al. (2000a) showed that transient phenomena interfere mass-loading signatures and dominate over them. It was shown that variations in the solar wind speed at $R \geq 6000 \mathrm{~km}$ are mainly related with propagating large-amplitude Alfvén waves. The flow and magnetic field perturbations are well correlated and follow Walén's relation $v= \pm b /\left(\rho^{1 / 2}\right)= \pm K_{\mathrm{A}} b$, where $\rho$ is the plasma mass density and \pm sign is taken as the sign of the product $-\left(k \cdot B_{0}\right)$ and varies in dependence on the wave propagation vector $k$ with respect to the ambient magnetic field $B_{0}$. Figure 13 show typical examples of relationship between the $v_{x}$ and $b_{x}$ components measured upstream of the bow shock. A linear dependence between the perturbations $v_{x}=$ $K b_{x}$ with the factor $K$ close to the Alfvén wave factor $K_{A}$ strongly suggests the wave origin of the observed perturbations. The in-phase relationship between the velocity and field perturbations in all these cases suggests the antisunward direction of wave propagation.

It is interesting to note that propagation of such waves explains decrease of the solar wind speed in the foreshock as compared to the velocity outside the foreshock. Figure 14 shows a scheme illustrating the effect of waves propagating antiparallel to the IMF in the antisunward direction. If the perturbation $b_{x}>0$, the tangent line (TL) turns from its nominal position shown by the thick arrow to the position (1). Then the observer that was in the solar wind close to the tangent line may occur in the foreshock and records a decrease of the speed $\left(\left|V_{0}+v\right|<\left|V_{0}\right|\right.$, $V_{0}<0, v>0$ ). For the perturbations with $b_{x}<0$ the tangent line goes to the position (2) and the observer occurs farther from the tangent line where the velocity increases. It was found that the observed variations in the flow can be provided 
a)
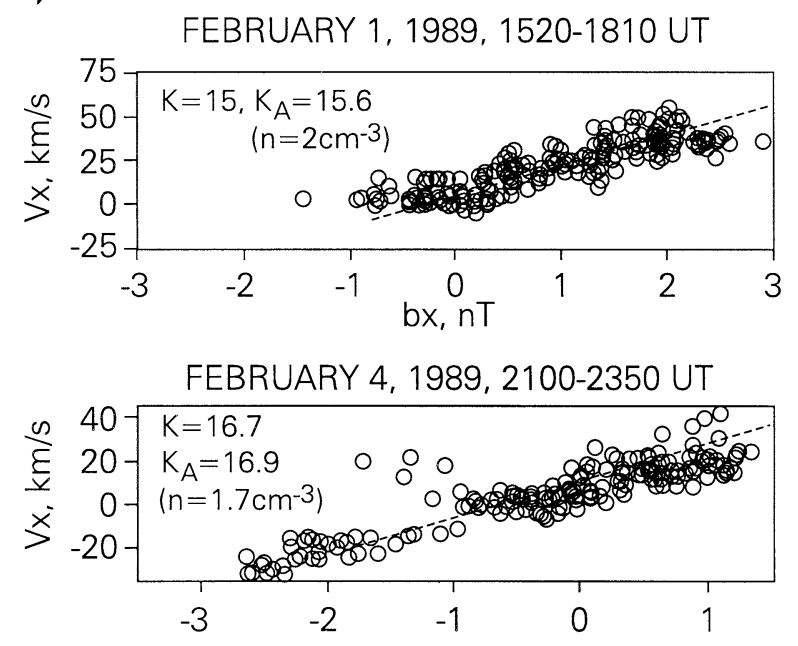

b)

bx, nT
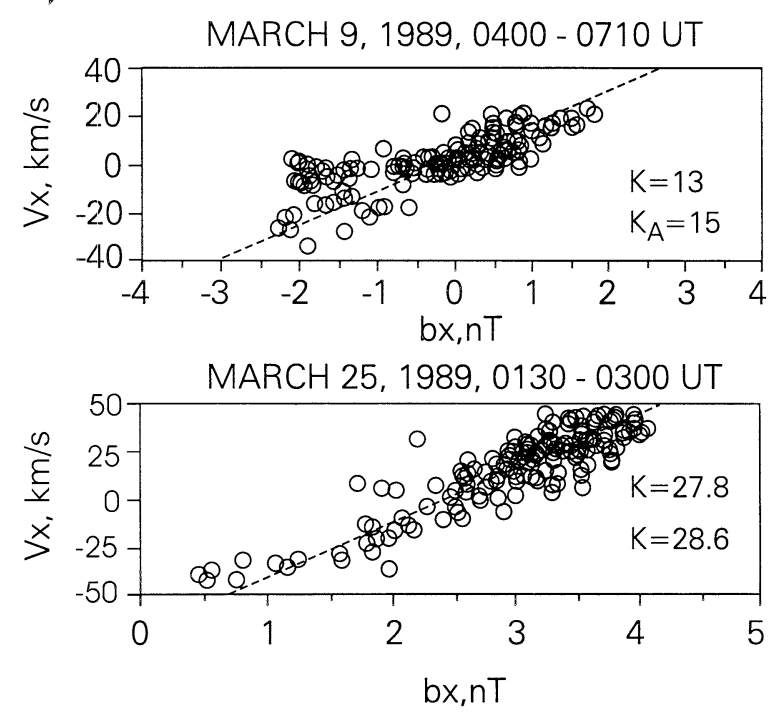

Figure 13. Relationship between variations in the speed $(V x)$ and the magnetic field $(B x)$ well follows the Walén relation for Alfvén waves propagating in the solar wind. At close distances to the bow shock perturbations in the flow and the magnetic field do not follow the Walén relation (not shown) (adapted from Dubinin et al., 2000a).

either by waves of the solar wind origin or by waves excited in the foreshock or near its boundary.

In all cases the foreshock boundary is not just a topological boundary separating the regions either magnetically connected or disconnected with the bow shock. Transition across the tangent line is often accompanied with a sharp rotation of the 


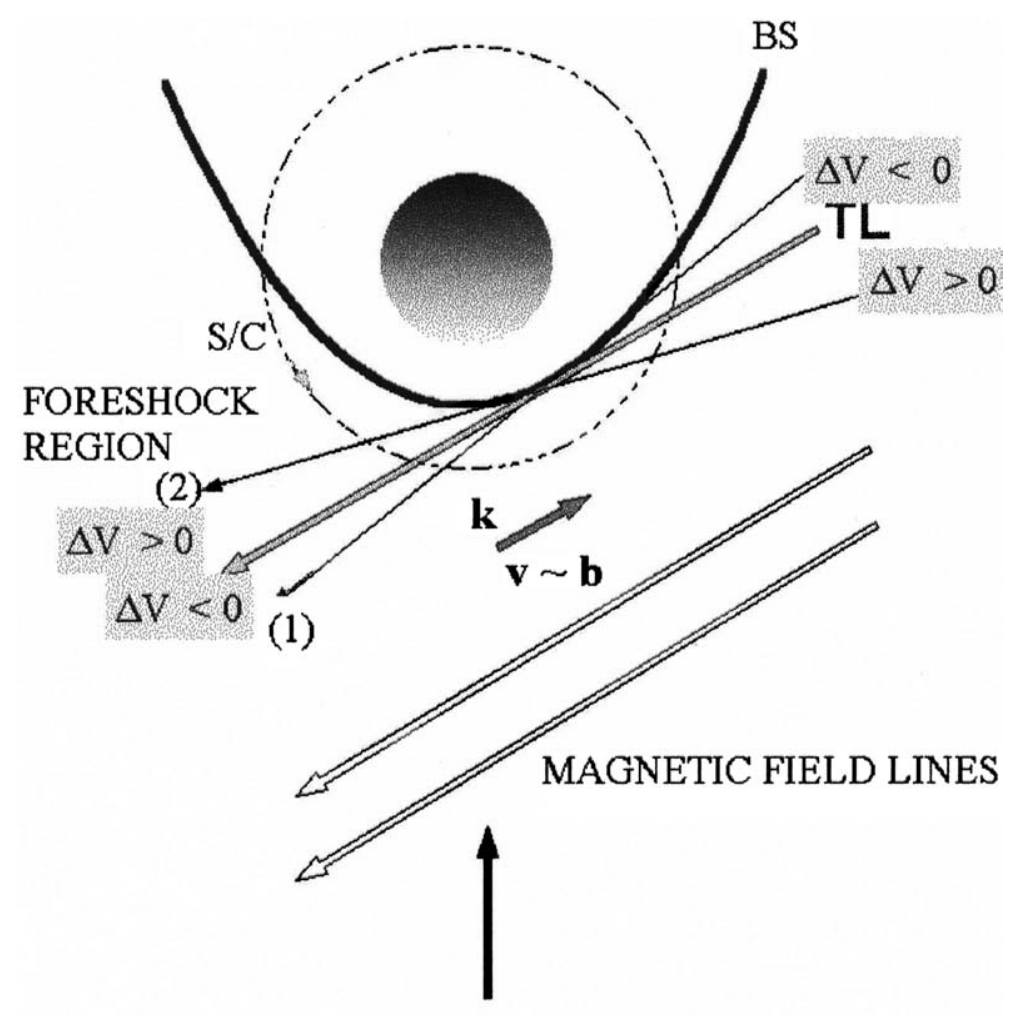

SOLAR WIND

Figure 14. Scheme illustrating the effect of Alfvén waves propagating in antisunward direction. For sunward propagation, the structure of the solar wind/foreshock interface is reversed in dependence on the direction of wave propagation (adapted from Dubinin et al., 2000a).

magnetic field, decrease in the field value and increase in the proton number density and temperature (Figure 15). Although variations of the solar wind speed at $R \geq$ $6000 \mathrm{~km}$ are mainly controlled by Alfvén waves their generation or amplification in the foreshock may be closely related with large fluxes of backstreaming ions.

At closer distances to the planet where the solar wind slows down at $\sim 100 \mathrm{~km} \mathrm{~s}^{-1}$ and the perturbations of the field and velocity do not follow the Walén relation, deceleration may be due to mass-loading on oxygen atmosphere or related with foot phenomena, e.g. with an enhancement of the number density of pickup ions in front of the bow shock due to multiple reflections of pickup ions. 
MARCH 25, 1989, 0130-0300 UT
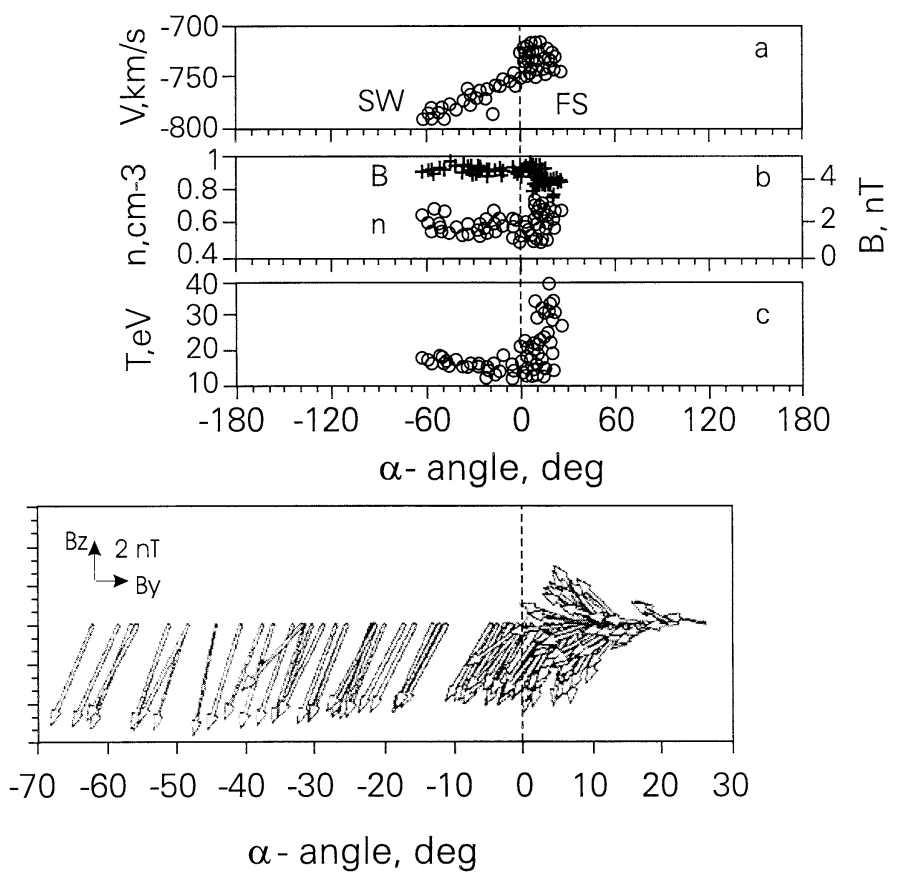

Figure 15. Variations in the bulk speed, the proton number density and proton temperature across the foreshock boundary along the circular orbit at $R=\sim 2.8 R_{M}$. Position of the spacecraft with respect the foreshock boundary is given in angle between the tangent line and the direction to Phobos-2 from the tangent point. Positive (negative) values correspond to the location of the spacecraft in the foreshock (solar wind). The bottom panel depicts projections of the magnetic field onto the YZ plane (the MSO reference frame) (adapted from Dubinin et al., 2000a).

\subsection{CHARACTERISTICS OF THE UPSTREAM FORESHOCK REGION}

\subsubsection{General Aspects of Foreshock Geometry}

The three-dimensional geometry of the foreshock is determined through the main motion of particles back-streaming from the bow shock. The foreshock region is magnetically connected to the bow shock and limited upstream by the magnetic field line tangent to the model bow shock. Particles back-streaming from the bow shock are guided by the magnetic field lines (limited by the tangent field line). Close to the tangent field line, only back-streaming electrons are observed (owing to their higher velocities) and form the electron foreshock, the (slower) back-streaming ions are found further downstream from the tangent field line, generating VLF and ULF waves. An overview of the wave observations in the Earth's foreshock is shown in Figure 16 (from Greenstadt et al., 1995). 


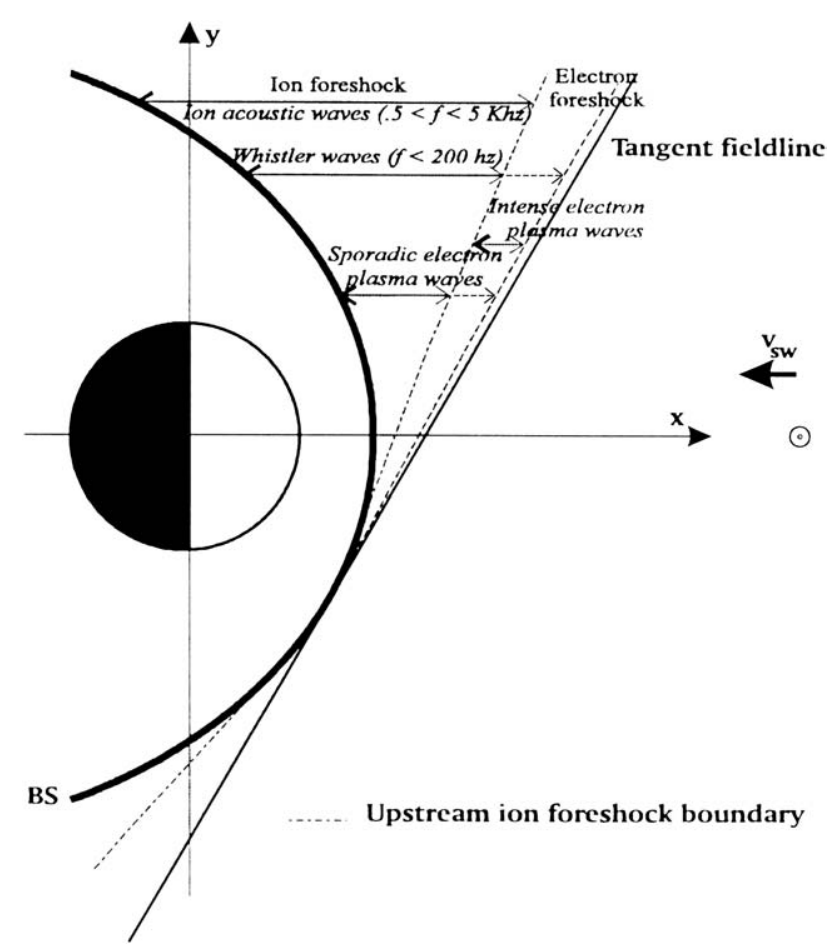

Figure 16. Wave modes, typical for the electron and ion foreshock at Earth (from Greenstadt et al., 1995).

\subsubsection{Mars Foreshock Results}

From the Phobos 2 data, the electron foreshock was observed through the appearance of 5.8-28 kHz emissions (Skalsky et al., 1993). Simultaneous electron measurements revealed enhanced fluxes of particles, back-streaming from the bow shock along the magnetic field. From the Phobos 2 data of multiple instruments (Dubinin et al., 2000a) the foreshock geometry was also well traced by a change of the antisunward/sunward anisotropy of suprathermal electron fluxes. More details about electric field waves will be given below.

The existence of the ion foreshock at Mars was stated from the Phobos 2 observations by Russell et al. (1990), based on the magnetometer data, and by Grard et al. (1991), based on the broadband electric noise upstream of the bow shock. This noise $(750 \mathrm{~Hz}-4 \mathrm{kHz})$ was interpreted as ion acoustic waves, with typically weak amplitudes and bursty in nature. Weak waves at the proton gyro frequency were observed upstream of the bow shock, associated with pick-up of protons from the Mars hydrogen exosphere (Russell et al., 1990).

Statistics from the circular orbits of the Phobos 2 S/C (March 1989) showed the following characteristics (Delva and Dubinin, 1998; Skalsky et al., 1998; Dubinin et al., 2000a): 
- Waves in the range of the electron plasma frequency are most intense close to the upstream boundary of the electron foreshock.

- ULF waves $(0.2-10 \mathrm{~Hz})$ gradually increase at larger distances from the tangent line, i.e., with the depth of the S/C location in the foreshock region.

- Deceleration of the solar wind starts closely after the crossing of the foreshock boundary, with significant deceleration closer to the bow shock, accompanied by a strong increase of the ULF turbulence.

- An enhanced level of electric field oscillations in the range $50-200 \mathrm{~Hz}$ is typically detected inside the foreshock.

- Intensities of the broadband electric emissions at frequencies of $0.5-4 \mathrm{kHz}$ are weak, but the most intense bursts occur deeper in the foreshock, compared to the higher frequency waves.

- For about $30 \%$ of the Phobos 2 S/C passes through the foreshock region, the onset of the waves with frequencies $50-200 \mathrm{~Hz}$ occurred upstream of the foreshock boundary.

- ULF waves are also observed upstream of the tangent field line; they might be of solar wind origin or generated by pickup protons and oxygen ions, originating from the extended hydrogen atmosphere and hot oxygen corona.

As was already discussed in the previous section, the multi-instrument observations from Phobos 2 showed that the foreshock boundary is not simply a topological boundary separating the regions magnetically connected to the bow shock (Dubinin et al., 2000a). Large amplitude Alfvén waves essentially contribute to the perturbations of the plasma and the IMF near the tangent line. Even for waves of solar wind origin, the transition across the tangent magnetic field line is often accompanied by a sharp change of the clock angle of the magnetic field, a decrease of the magnetic field value and an increase of the proton number density and temperature. Downstream of the upstream foreshock boundary, ULF waves are generated by back-streaming ions and ions of planetary origin interacting with the on-streaming solar wind.

From the Mars Global Surveyor S/C data, it is not always possible to distinguish if the $\mathrm{S} / \mathrm{C}$ is located in the ion foreshock or not, due to a lower accuracy of the magnetometer data of the MGS MAG sensors, when the S/C is in the solar wind and the solar panels are illuminated (Acuña et al., 2001). The obtained accuracy of $\sim 1 \mathrm{nT}$ prevents from directly using the MAG data to study the connection of the local field line to the bow shock when the ambient IMF magnitude is small, which is often the case at Mars. For orbits for which the IMF is larger than 4$5 \mathrm{nT}$, typically, it is possible to determine the tangent field line with high enough accuracy and check whether the spacecraft is in the foreshock or not. Since there was no wave instrument on MGS, only low frequency electromagnetic waves were accessible to observations.

Low frequency waves at the proton cyclotron frequency were frequently observed upstream of the bow shock (Brain et al., 2002) and often appear as large amplitude highly coherent waves (Mazelle et al., 2001). On MGS orbits for which 


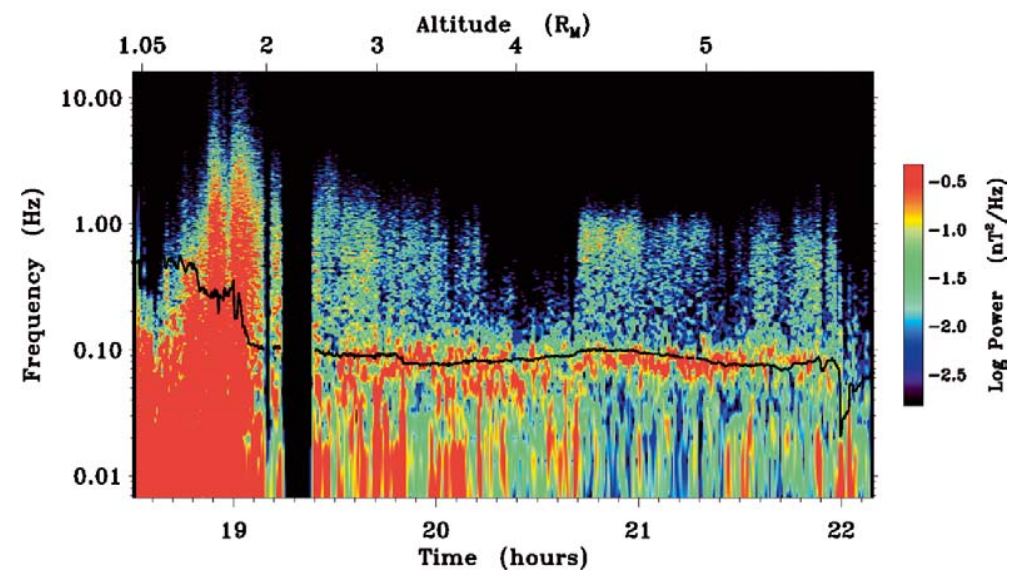

Figure 17. Time-frequency spectrogram of one vector field component of MGS MAG data from April 24, 1998 (Brain et al., 2002). Two wave features are evident - one at the local proton gyrofrequency (plotted over the spectrogram in black), and one near $1 \mathrm{~Hz}$. A bow shock crossing occurs at $\sim 19.1$ hours, after which MGS is outside of the shock. The data were rotated into mean-field coordinates (with the mean field pointing in the $+z$ direction) before the spectrogram was applied.

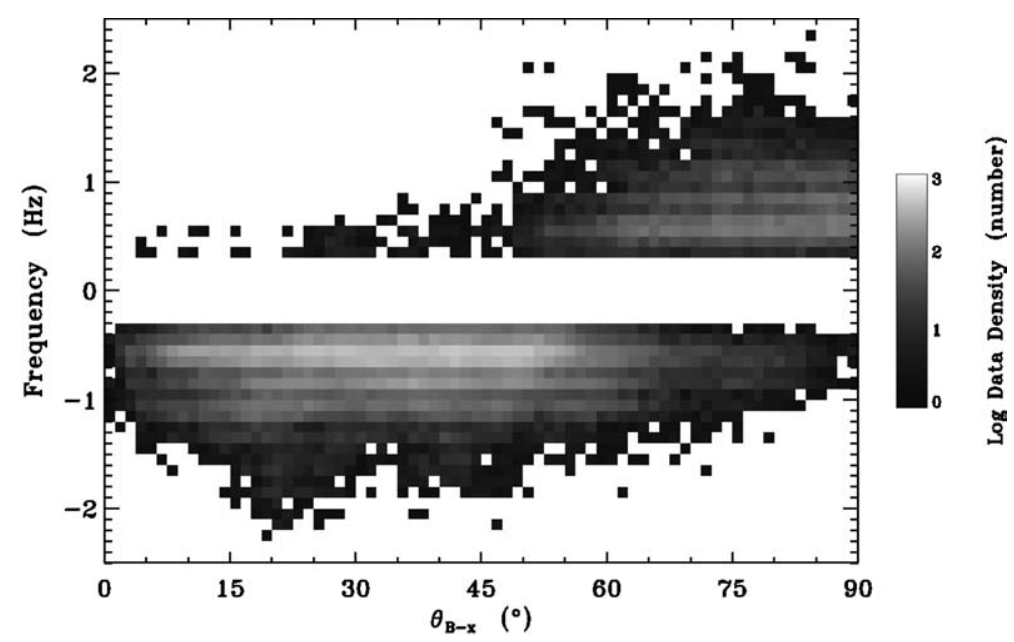

Figure 18. Frequency of whistler waves observed by MGS MAG as a function of the angle between the magnetic field and the solar wind velocity (Brain et al., 2002). The solar wind velocity is taken in the $x$ direction. Negative frequencies indicate left hand polarization in the rest frame of the spacecraft.

the foreshock boundary can be determined from the tangent field line, such waves are observed with the same characteristics for a large range of radial distances and even when the spacecraft is not magnetically connected to the bow shock (Mazelle et al., 2001). This indicates that they are not ion foreshock-related, i.e., not associated to back-streaming ions. Moreover, the orbits for which coherent monochromatic upstream waves at the proton cyclotron frequency are observed, never show a sharp interruption of the waves in the time series of the magnetic 
field as the S/C leaves the foreshock region, although this is characteristic for observations at the Earth, when a S/C has crossed the ion foreshock boundary. These wave observations will be detailed below.

Higher frequency $(\sim 1 \mathrm{~Hz})$ whistler waves were also detected in the MGS magnetometer data upstream of the Martian bow shock, which were not reported from the Phobos 2 data. They occurred mainly while MGS was magnetically connected to the bow shock, i.e., they appear to be foreshock phenomena (Brain et al., 2002).

\subsection{OBSERVATIONS OF LOW FREQUENCY WAVES}

The magnetometer/electron reflectometer (MAG/ER) on board the Mars Global Surveyor (MGS) has recorded a variety of plasma wave oscillations in the magnetic field throughout the Martian plasma environment (Mazelle et al., 2000, 2001; Brain et al., 2002; Cloutier et al., 1999; Crider, 1999). Two distinct wave frequencies are often observed upstream from the shock (Figure 17). The elliptical orbit used in the pre-mapping portion of the mission allowed substantial altitude and solar zenith angle coverage of the region upstream from the bow shock, ranging from $\sim 30$ 150 in SZA and out to $\sim 17 R_{M}$ for some orbits. Data is returned at 32, 16, or 8 samples per second, corresponding to Nyquist frequencies of $16 \mathrm{~Hz}, 8 \mathrm{~Hz}$, or $4 \mathrm{~Hz}$. A lower time resolution version of the data is available (with Nyquist frequencies of $0.17-0.67 \mathrm{~Hz}$ ), and has the advantage of having magnetic field contributions from the spacecraft and solar panels removed (lack of high time resolution engineering data from the spacecraft make calibration impractical for the high time resolution MAG data). The calibrated data are accurate to $\sim 1 \mathrm{nT}$ in sunlight, and $\sim 0.5 \mathrm{nT}$ in shadow (Acuña et al., 2001). Fortunately, the remaining spacecraft contributions to the magnetic field are due to the thermal response of the solar panels, which occurs at very low frequencies and can be filtered out of the data when searching for plasma waves. However, the direction of the ambient magnetic field is uncertain to within the accuracy of the calibration.

A recurring wave feature in the frequency range $0.4-2.3 \mathrm{~Hz}$ is evident in high time resolution data upstream from the shock (Brain et al., 2002). This feature has many characteristics that are consistent with whistler waves observed at other solar system bodies, including Earth, Mercury, Venus, and Saturn (Orlowski et al., 1990, 1992). First, the waves are associated with the foreshock. Second, the polarization sense for the waves is controlled by the angle between the ambient magnetic field and the solar wind flow (Figure 18). Third, the amplitude of the wave decreases with distance to the shock (along the magnetic field line). Fourth, the wave characteristics of Martian whistlers follow trends with heliocentric distance observed for whistlers at other solar system bodies. These trends include a decrease in frequency and amplitude with heliocentric distance, and an increase in eccentricity and the angle between the wave propagation vector and background magnetic field $\left(\theta_{k B}\right)$ (Orlowski and Russell, 1995). 
It is believed that the whistlers are generated at or near the shock, and propagate upstream with a group velocity greater than the solar wind velocity of $\sim 400 \mathrm{~km} \mathrm{~s}^{-1}$ (Fairfield, 1974). The direction of the ambient magnetic field determines the direction of propagation of the wave, but the component of the wave's group velocity in the direction of the solar wind flow will be Doppler shifted when the waves are observed in the rest frame of the spacecraft or planet. The whistlers are right handed in the plasma rest frame, but are observed as left hand waves when the angle between the background field and solar wind flow is low because of this Doppler shift. There are many open questions concerning upstream whistler waves (see Brain et al., 2002, and references), some of which may be answered by future spacecraft to Mars. A wide variety of generation mechanisms have been proposed, and it is still not clear whether ions can be responsible for some of the observed waves, and how the waves are damped.

Left-hand circularly polarized waves (PCWs) at the proton cyclotron frequency were first observed by Russell et al. (1990), using magnetometer data from the Phobos spacecraft. These waves were observed 2-3 $R_{m}$ from Mars on three spacecraft orbits, had low amplitude, and propagated at small angle to the background magnetic field. ASPERA observations of proton ring distributions in the hydrogen corona (Barabash et al. 1991), and lack of similar wave observations at Venus (which does not have an exosphere that extends beyond the shock) led Russell et al. (1990) to conclude that the waves were formed by solar wind pickup of newlyionized exospheric hydrogen. The highly eccentric orbit of MGS enabled confirmation of the Phobos observations, and examination of the spatial distribution of PCWs outside of the Martian shock.

Over 500 orbits of pre-mapping MAG data were analyzed for the presence of PCWs (Brain et al., 2002). The wave characteristics agreed with Phobos results in the observed frequency, eccentricity, and propagation angle, while the amplitudes recorded for MAG data were a factor of 2-3 higher than observed by Phobos. There is no correlation of wave observations with the foreshock, and the wave features are often long-lived in the data. The spatial distribution of power at the local proton gyrofrequency in MGS MAG data is shown in Figure 19. Wave power generally decreases with increasing altitude, and there are two spatial locations where PCWs are common. Waves near the subsolar point have lower amplitudes, lower eccentricities, and smaller values of $\theta_{k B}$ than waves observed near the flanks of the solar wind interaction. Wave power near the flanks decreases rapidly with altitude relative to waves near the subsolar point.

The analysis of MGS data by Brain et al. (2002) was based on a search for waves in specific frequency ranges. There is no reason that all observed whistler waves should be caused by a single mechanism, and the same is true for PCWs. It is even possible that the two different wave frequencies are related to each other for some of the observations. We note however, that this is not likely to be true for the majority of the observed upstream waves. First, given the constraints of the calibration to the data, the observed whistler waves are overwhelmingly associated 


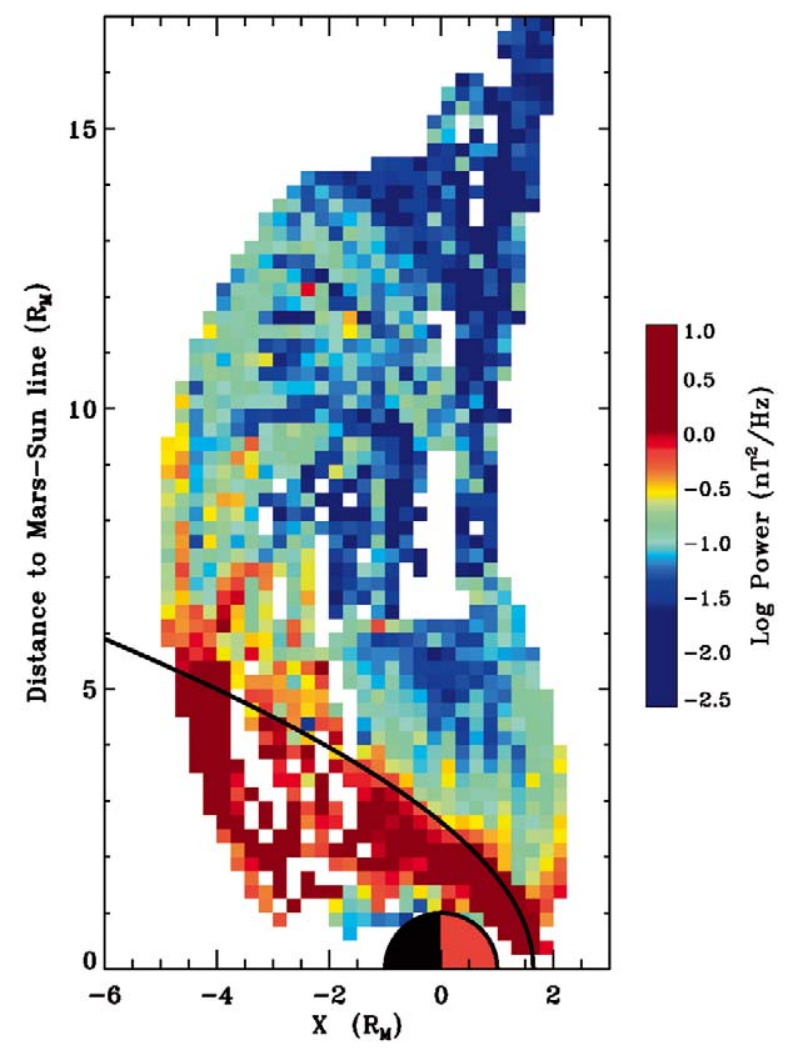

Figure 19. Spatial distribution of power at the local proton gyrofrequency in MGS MAG data (Brain et al., 2002). Average power is shown as a function of location for the $x$ component of magnetic field in mean-field coordinates. The best fit bow shock (Vignes et al., 2000) is indicated in black.

(88\%) with the foreshock, while the PCWs are not. Second, the occurrence of the two wave signatures is statistically independent of each other: whistlers are observed $18 \%$ of the time, PCWs are observed $26 \%$ of the time, and the two are observed concurrently $6 \%(\approx 0.26 \times 0.18)$ of the time.

Finally, large amplitude, highly coherent upstream waves at the proton cyclotron frequency $f_{c}$ were reported by Mazelle et al. (2001). These waves are observed for a large range of radial distances and even when the spacecraft is not magnetically connected to the bow shock; therefore, they are not ion foreshock-related, i.e., not associated to back-streaming ions. The observed periods (spacecraft frame) always closely fit the local proton cyclotron periods. The waves are left-handed circularly polarized in the spacecraft frame. Their wave vectors lay at moderate angles from the ambient magnetic field. Thus, at first glance, such waves would be as usual interpreted as generated by a resonant helical beam instability fed by the planetary pick-up protons, which can lead to derive constraints on the hydrogen density in the Martian exosphere. 

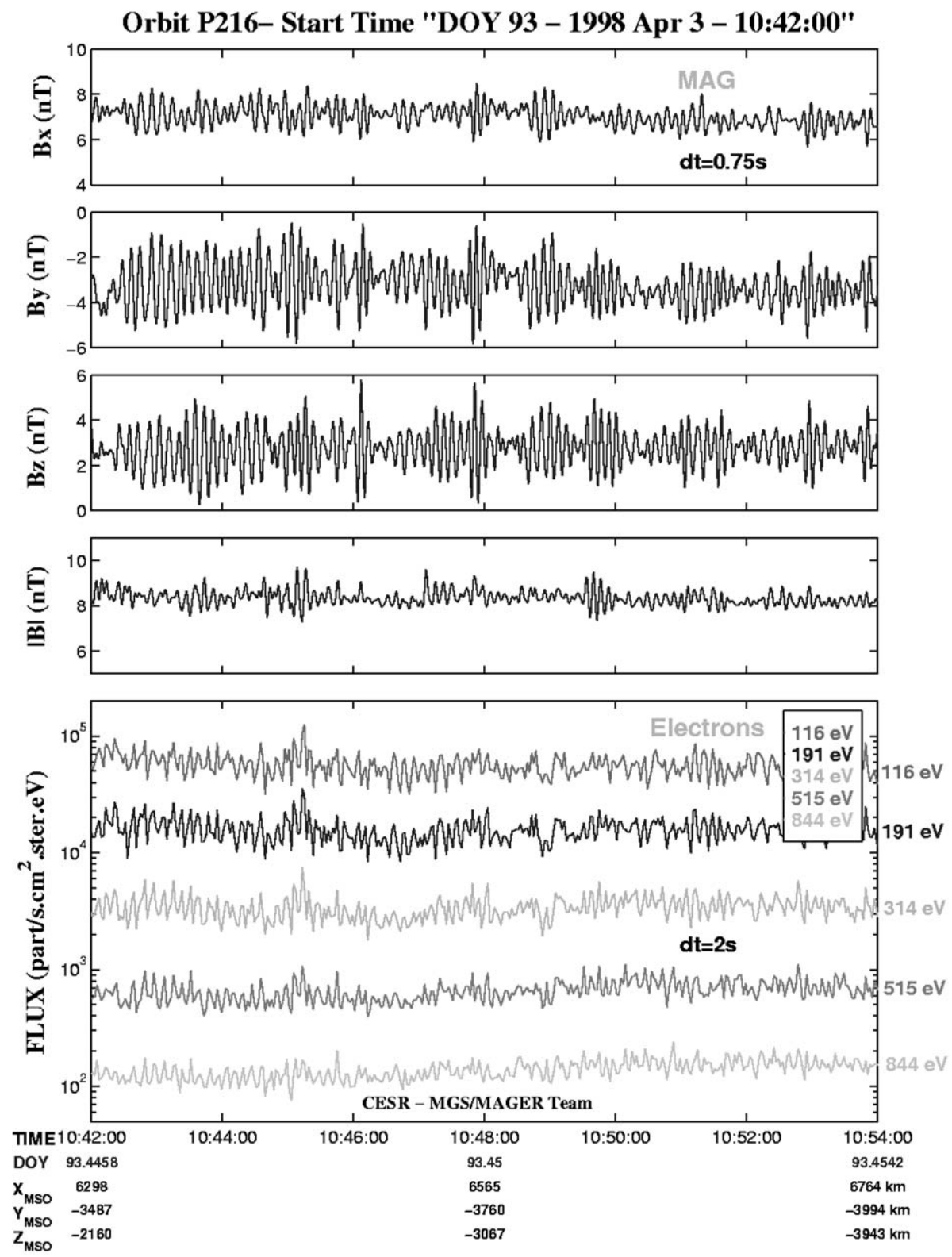

Figure 20. Examples of highly coherent wave packets with oscillating substructure ('oscillitons') reported from observations by Mars Global Surveyor upstream from the bow shock (from Mazelle et al., 2002). The 'high' frequency sinusoidal signature is exactly at the local proton cyclotron period while the lower frequency 'envelope' curve is about at 7 gyroperiods. 
However, these 'proton cyclotron waves' are frequently revealed as monochromatic wave packets embedded inside a regular lower frequency 'wave envelope' at about $f_{c} / 10$ (Figure 20). They can have large amplitude (up to $5 \mathrm{nT}$ peak-to-peak) even at large distance from the planet (more than $6 R_{M}$ ). Their amplitude is too large to be consistent e.g., with the saturation value for the picked-up proton ring instability using realistic neutral densities from theoretical models of the Martian exosphere (e.g., Kim et al., 1998). Moreover, a satisfactory mechanism is also needed for both the lower frequency 'envelope' signature and the high coherence of the ion gyrofrequency signature. Classical nonlinear processes occurring for finite amplitude circularly polarized Alfvén waves such as the decay or the modulational instability can be excluded (e.g., Spangler et al., 1988, and references therein). The oscillations of the electron fluxes for all energy channels (and thus the density) are in close correlation with the magnetic field wave oscillations whereas ponderomotive effects for a circularly polarized Alfvén wave would make the density perturbation to be proportional to the square of the wave amplitude and thus be observed at half the wave period (Spangler et al., 1988). These waves can be interpreted as oblique bi-ion stationary waves ('oscillitons') (Sauer et al., 2001, 2002a). This will be detailed in Section 4.6. They can be produced (in the flow regimes in which they are permitted) by the existence of two ion populations (solar wind + planetary) with different densities, velocities and/or temperatures. This implies that direct connection between wave amplitude at the cyclotron frequency and neutral densities in the planetary exosphere is not always ensured.

\subsection{OBSERVATIONS OF HIGH FREQUENCY PLASMA WAVES}

The bow shock is usually considered to be crossed when the ramp in the magnetic field is encountered. As this shock transition region separates the upstream region, where charged particles energized and reflected at the shock cause a variety of instabilities and attendant waves, from the downstream region, in which the wave activity fades away after reaching a maximum at the ramp, bow shock crossings are also clearly identified from plasma and wave data. This point is illustrated in Figure 21, which displays some of the parameters measured with the plasma wave system on February 11, 1989, along the fourth elliptical orbit of Phobos 2 around Mars: from top to bottom, (1) the r.m.s. fluctuation of the current collected with the Langmuir probe, (2) the high frequency variation of it, and (3) the electric field intensity measured by the $1.45 \mathrm{~m}$ long double sphere antenna as a function of time and frequency, from $5 \mathrm{~Hz}$ to $150 \mathrm{kHz}$. For a full description of the plasma wave system, the reader is referred to Grard et al. (1989). The color scaling shown on the right side of Figure 21 depends on the strength of the electric field, which is expressed in decibels above $1 \mu \mathrm{V} \mathrm{m}^{-1} \mathrm{~Hz}^{-1 / 2}$, so that $60 \mathrm{~dB}$ correspond to a signal level of $1 \mathrm{mV} \mathrm{m}^{-1} \mathrm{~Hz}^{-1 / 2}$. A vertical dashed line marks the shock ramp, which was crossed at 11:03 UT. The abrupt burst of the electric field level, from a few $\mathrm{Hz}$ up to $10 \mathrm{kHz}$, the jump in the electron density, which is seen as a jump 

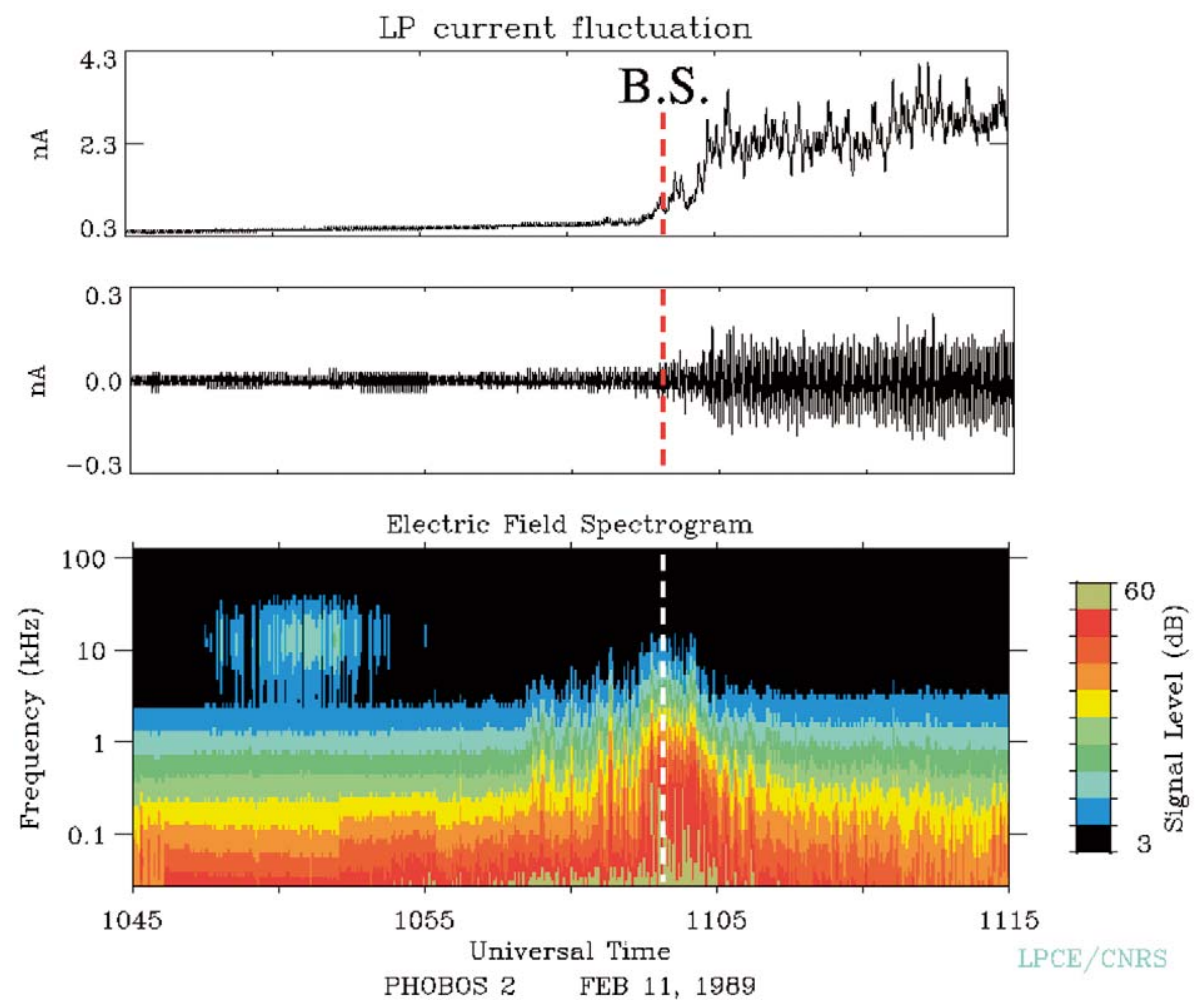

Figure 21. From top to bottom: root-mean-square of the current collected with the Langmuir probe, high frequency variation of this signal, and dynamic electric field spectrogram measured by the plasma wave system onboard Phobos 2. The Martian bow shock was crossed at 11:03 UT on February 11, 1989 (vertical dashed line). Electron plasma oscillations occurred at about $13 \mathrm{kHz}$ in front of the shock (Trotignon et al., 1993.

in the Langmuir probe current fluctuation, as well as the increase of the plasma turbulence just behind the shock transition region allow a reliable and accurate location of the shock front to be determined.

The electric field spectrum recorded by PWS in the Martian shock ramp at 11:02:42 UT on February 11, 1989, is plotted as crosses in Figure 22. In addition, several plasma characteristic frequencies, the electron cyclotron frequency $\left(F_{c e}\right)$, the ion plasma frequency $\left(F_{p i}\right)$, the Buneman frequency $\left(F_{B}\right)$, the upper frequency cut-off of Doppler-shifted ion-acoustic waves $\left(V_{S W} / 2 \pi \lambda_{D}\right.$, where $V_{S W}$ is the solar wind velocity and $\lambda_{D}$ the plasma Debye length), and the local plasma frequency $\left(F_{p e}\right)$ are pointed out by arrows. Similar to the Earth's bow shock (Grard et al., 1989, 1993; Trotignon et al., 1991a) there is evidence that two well-defined wave components are present in the shock transition region. The 10:02:42 UT electric field spectrum shown as crosses in Figure 22 indeed consists of a low frequency component below $F_{c e}$ and a high frequency one with an apparent frequency cutoff at $V_{S W} / 2 \pi \lambda_{D}$. The low-frequency noise is attributed to the electric component 


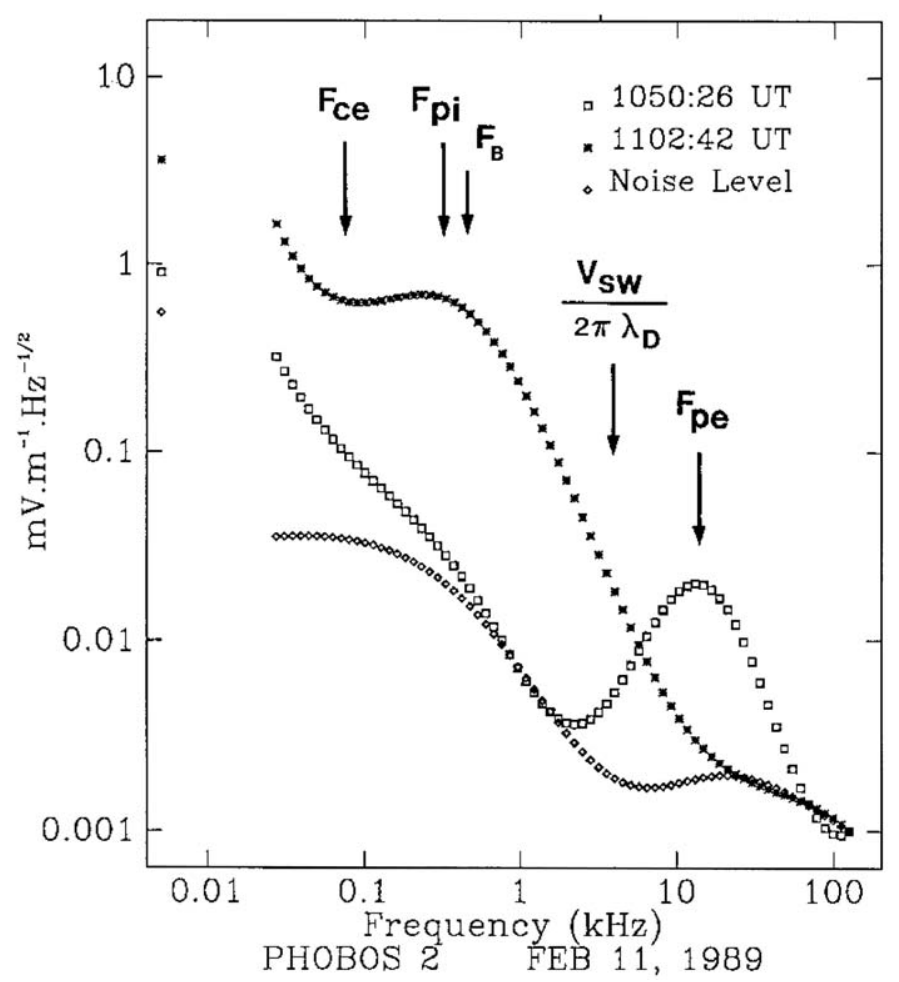

Figure 22. Electric field spectra recorded by the plasma wave system onboard Phobos 2 in the free solar wind (small diamonds), in the Mars' electron foreshock (squares) where electron plasma oscillations were observed, and in the shock ramp (crosses). Plasma characteristic frequencies are indicated by vertical arrows (Trotignon et al., 1991c).

of the electromagnetic whistler mode emission, while Doppler-shifted ion-acoustic waves, which are electrostatic in nature, are thought to be responsible for the high frequency spectral component (Sagdeev et al., 1990; Grard et al., 1991; Trotignon et al., 1991c). Consequently, the whole frequency range explored by the PWS has been split into three consecutive bandwidths: (1) the $25 \mathrm{~Hz}-100 \mathrm{~Hz}$ low-frequency bandwidth for whistler mode emissions, (2) the $100 \mathrm{~Hz}-6 \mathrm{kHz}$ middle frequency bandwidth to cover the ion-acoustic Doppler-shifted frequency range, and (3) the $6 \mathrm{kHz}-130 \mathrm{kHz}$ very high frequency bandwidth for the upstream electron plasma waves, which will be introduced ahead. The result of the splitting process applied to the Figure 21 time interval is presented in Figure 23. A peak is observed in the wave intensity, in particular in the middle frequency bandwidth, which covers the ion-acoustic Doppler-shifted frequency domain.

The cluster of intense noise bursts shown in the top panel of Figure 23 is centered on $13 \mathrm{kHz}$. These wave bursts, also present in the color spectrogram of Figure 21 are usually observed in front of the Martian shock whenever the interplanetary magnetic field line that passes through the spacecraft position intersects 

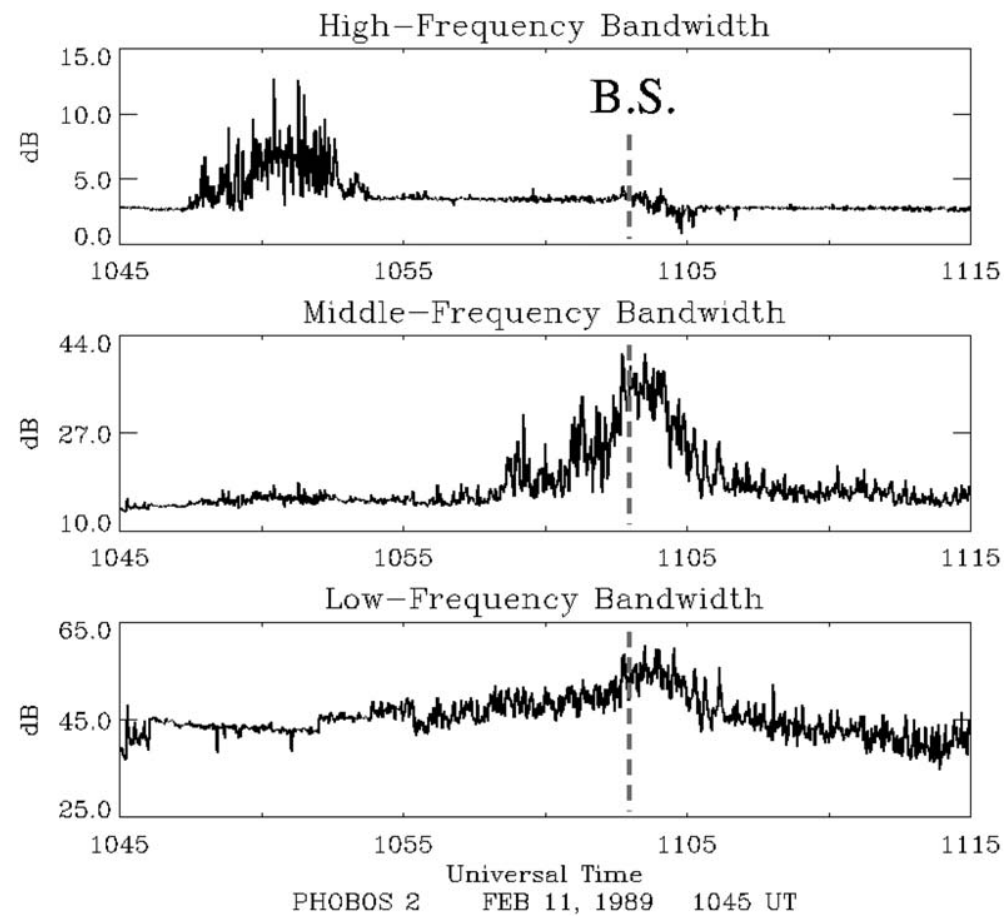

Figure 23. Intensity of the electric field averaged over three frequency bandwidths: $25-100 \mathrm{~Hz}$ (bottom), 100-6 kHz (middle), and 6-130 kHz (top). The bow shock has been identified from these Phobos 2 plasma wave system measurements at 11:03 UT, on February 11, 1989. Electron plasma waves are seen as an electric intensity increase in the high frequency bandwidth (Trotignon et al., 1993).

the shock front. Such waves have commonly been reported upstream of most of the solar system planets and even some comets. These electrostatic waves, also called electron plasma oscillations, are known to be generated by a two-stream plasma instability in the electron foreshock region, where solar wind electrons that are energized and accelerated at the shock front stream back into the solar wind along the interplanetary magnetic line connected to the shock.

A typical spectrum of these electron plasma oscillations is plotted in Figure 22. This spectrum (squares) detected at 10:50:26 UT on February 11, 1989, exhibits a bump at or close to the solar wind plasma frequency, $F_{p e}$, from which the solar wind density may directly be deduced (Trotignon et al., 1992): at this time $F_{p e}$ and $N_{e}$ were $14 \mathrm{kHz}$ and $2.4 \mathrm{~cm}^{-3}$, respectively. As can be seen in Figure 22, the electron plasma oscillation level is more than one order of magnitude higher than the noise level recorded by the PWS in the unperturbed solar wind (small diamonds). Skalsky et al. (1992) reported that the observed electron plasma oscillations were polarized along the magnetic field, i.e., longitudinal, as expected for such upstream electron plasma wave in the electron foreshock. Large electron flux anisotropies were detected by the ASPERA experiment in association with 
the PWS observations (Barabash et al., 1993). Finally, the electric field emissions turned out to be associated with flux enhancements of electrons with energies from $100 \mathrm{eV}$ to $530 \mathrm{eV}$ that were backstreaming into the solar wind (Skalsky et al., 1993).

The electron plasma oscillation occurrence and characteristics depend on connection parameters calculated in the so-called $\mathbf{B}-\mathbf{v}$ plane, where $\mathbf{B}$ and $\mathbf{v}$ are the interplanetary magnetic field and solar wind speed vectors, respectively. Some of them are displayed in the top panels of Figure 24, between 22:35 UT and 23:35 UT, on February 4, 1989, when Phobos 2 crossed the electron foreshock region along its second elliptical orbit around Mars. The shock subsolar distance is the distance from the center of Mars to the nose of the shock surface section. The connection depth is the distance from the spacecraft to the interplanetary magnetic field line that is tangent to the shock surface section. This distance measured parallel to $\mathbf{v}$ is positive whenever the spacecraft is located downstream of the tangent field line. The time of flight distance is the distance between the point of tangency and the spacecraft position, i.e., the distance covered by the supra-thermal electrons that are reflected at the tangent point. The distance covered by the electrons that stream back along the magnetic field line that intersects the spacecraft position is called the connection distance. Finally, the angle between the normal to the shock front and the spacecraft field line is the $\theta_{B n}$ angle.

The top panel of Figure 24 thus shows that the shock subsolar distance increases regularly, i.e., the shock surface section appears to move regularly from the antisunward direction to the sunward direction until the mean shock subsolar distance of $1.57 R_{M}$ obtained by Trotignon et al. (1993) is reached (solid horizontal line). In the meantime, the interplanetary magnetic field line that passes through the spacecraft position is sometimes connected to the shock, sometimes not. When the field line is connected, the connection distance and $\theta_{B n}$ may be calculated. Conversely, when it is not, the two parameters cannot be derived. It becomes consequently easy to see in Figure 24 that the electron plasma oscillations shown in the color spectrogram only occur when the connection takes place, i.e., when the connection distance and $\theta_{B n}$ angle are plotted.

Assuming that the interplanetary magnetic field line is at a $57^{\circ}$ angle to the subsolar direction, i.e., the nominal Parker interplanetary magnetic field orientation, a statistical image of the Mars' electron foreshock region may be constructed. In this image, the electric field intensity averaged over the $6 \mathrm{kHz}-130 \mathrm{kHz}$ frequency bandwidth is plotted as a function of the equatorial $\mathbf{B}-\mathbf{v}$ plane coordinates, i.e., the plane that is parallel to the $\mathbf{B}-\mathbf{v}$ plane and passes through the planet center. As can be seen in Figure 25 borrowed from Trotignon et al. (2000), the highest electric field levels, which correspond to electron plasma waves in the electron foreshock of Mars, are observed in a limited region behind the tangent field line. Figure 25 also shows that no instabilities and therefore electron plasma oscillations arise beyond $6 R_{M}$ from the point of tangency. The small size of the Martian shock 

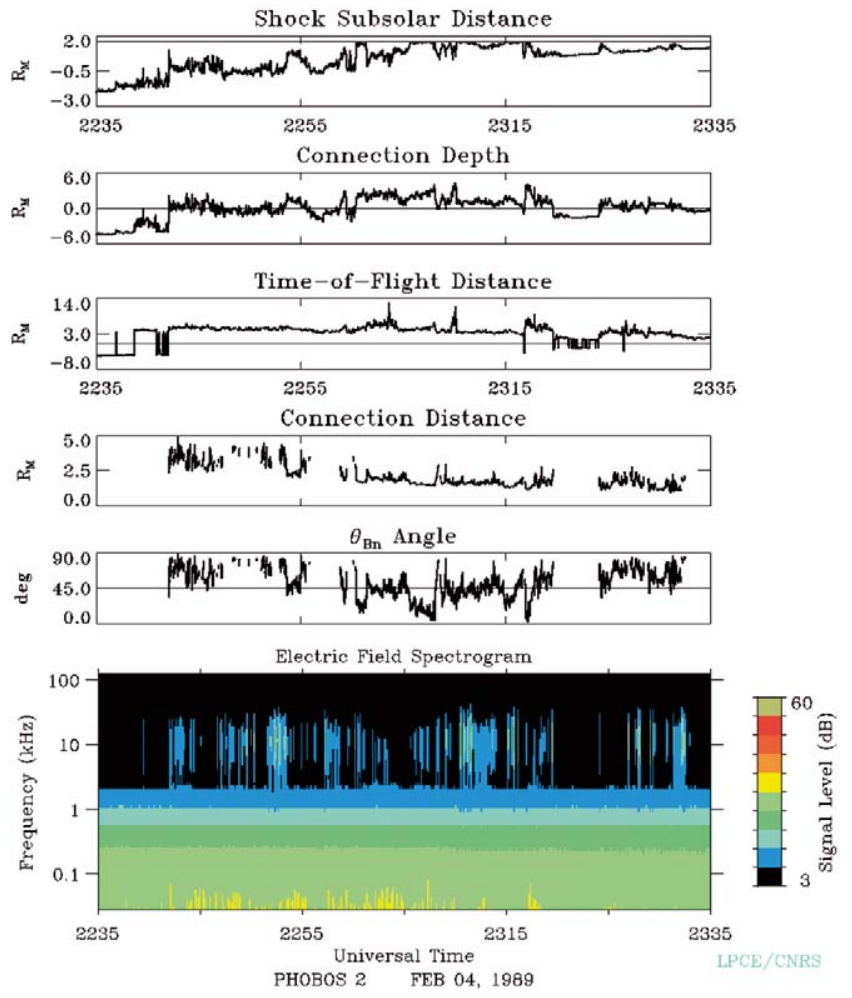

Figure 24. Electric field spectrogram measured by the plasma wave system in the electron foreshock of Mars along the second elliptical orbit of Phobos 2 around the planet (bottom) and connection parameters calculated in the $\mathbf{B}-$ $\mathbf{v}$ plane, where $\mathbf{B}$ and $\mathbf{v}$ are the interplanetary magnetic field and solar wind velocity vectors, respectively (Trotignon et al., 2000).

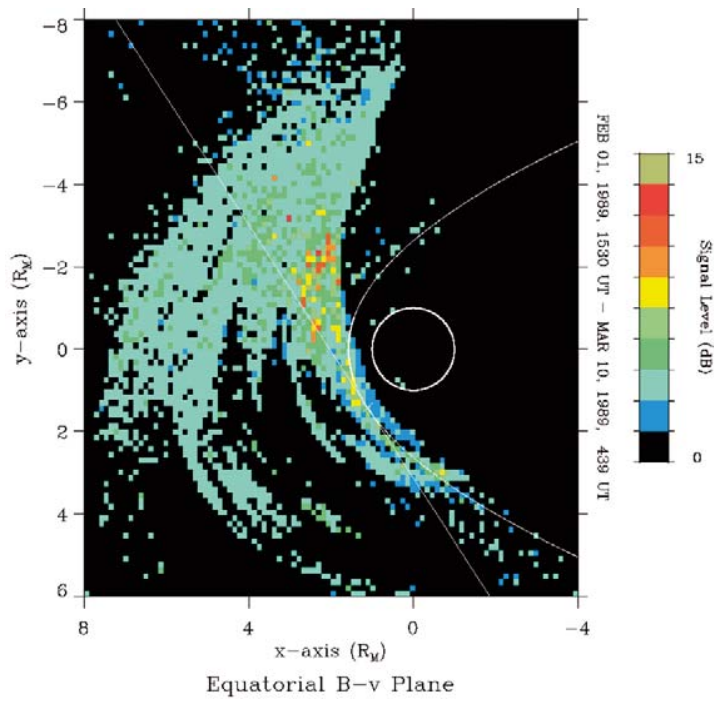

Figure 25. Statistical image of the electric field intensity integrated in the $6 \mathrm{kHz}-130 \mathrm{kHz}$ frequency bandwidth measured by the Phobos 2 plasma wave system. $(\mathrm{X}, \mathrm{Y})$ are the nominal equatorial $\mathbf{B}-\mathbf{v}$ plane coordinates. The Martian bow shock section and the nominal Parker interplanetary magnetic field line tangent to the shock are shown as white conic curve and straight line, respectively. The highest intensities are seen in the electron foreshock region, as expected (Trotignon et al., 2000). 
is thought to be responsible for this limited extension along the tangent field line by limiting the electron energization processes (see for example, Strangeway and Crawford, 1995).

\section{Sources of Low-Frequency Waves Upstream from the Bow Shock}

Low-frequency electromagnetic wave phenomena observed by Phobos 2 and MGS outside of the Martian bow shock include waves near the local proton gyrofrequency (PCWs), waves in the range of whistler frequencies ('upstream whistlers') and strongly localized wave-like solar wind perturbations (e.g., 'Phobos-Deimos events'). The presence of these different wave types support the suggestion that the Martian interaction with the solar wind is unique among the other planets. Whereas upstream whistlers have been observed at many other solar system bodies (Mercury, Venus, Earth, Saturn), the presence of proton gyrofrequency waves, although observed under several circumstances in space plasmas, seems to be a special, permanent feature of the Martian environment. There is general consensus that the PCW's can be attributed to the pick-up process of protons from the extended hydrogen corona of Mars. The significant localized wave-like solar wind perturbations, detected in the neighborhood of Mars, are somewhat exceptional and originate likely from the two Martian moons Phobos and Deimos rather than from Mars itself. The generation mechanism of any of the above three wave phenomena is still under debate and several scenarios have been introduced in the discussion.

\subsection{UPSTREAM WHISTLERS}

Upstream whistlers (or '1-Hz-waves') have long been known from Earth (Russell et al., 1971; Fairfield, 1974) and other planets (Orlowski and Russell, 1995) but were first detected at Mars by MGS (Brain et al., 2002). They are characterized by a propagation direction oblique to the magnetic field and by distinct polarization properties which tend to change from left-hand polarization to right-hand polarization with increasing propagation angle. The same tendency has been found with increasing heliocentric distances as a consequence of the varying spiral angle of the IMF (always left-handed at Mercury, about half the time left-handed at Earth, always right-handed at Saturn). The characteristics of upstream whistlers at Mars (frequency range $0.4-2.3 \mathrm{~Hz}$ ) are consistent with observations at other planets: their frequency and amplitude decreases with increasing angle between propagation direction and solar wind flow direction and their polarization properties are as expected for the heliocentric distance of Mars. Although comprehensive observational material has been accumulated on upstream whistlers, their generation mechanism is still not well understood. It is commonly accepted that the shock or the shock ramp is the location of generation and that the waves propagate upstream due to their large group velocity, guided by the magnetic field. The similarities 
between the waves observed at different planets suggest that the source mechanism is not sensitive to details of size and shape of the shock and that similar plasma processes are at work to produce the waves.

\subsection{WAVES AT THE PROTON GYROFREQUENCY}

Waves at the proton gyrofrequency (PCWs) have been previously observed at comets (Tsurutani et al., 1989; Mazelle and Neubauer, 1993), in the AMPTE experiment (Gleaves et al., 1987) and in the high-altitude cusp (Le et al., 2001), sometimes as monochromatic wave packets. At Mars PCWs were detected by both the Phobos 2 (Russell et al., 1990) and the MGS spacecraft (Brain et al., 2002). The MAG data from MGS, based on hundreds of orbits, provide comprehensive material to analyze the characteristics of the waves and to determine their spatial distribution with respect to Mars. The main features are: PCWs around Mars are always lefthand elliptically polarized and propagate at small to moderate angles to the ambient magnetic field $\left(\theta_{k B} \approx 20^{\circ}\right)$, in agreement with Phobos 2 observations, but have 23 times higher amplitude than the very low one $(\approx 0.15 \mathrm{nT})$ observed by Phobos. The wave intensity varies spatially, is concentrated near the subsolar point and the flanks of the solar wind interaction, and decreases with altitude. In the following we discuss several potential generation mechanisms of PCWs.

\subsubsection{Ring-Beam Instabilities}

The PCWs observed at Mars are generally believed to result from the pick-up process of protons from the hydrogen corona of Mars which extends beyond the Martian bow shock (Russell et al., 1990; Barabash et al., 1991). Pick-up processes have also been favored to explain PCWs in the cometary environment (Mazelle and Neubauer, 1993). At a first look it seems likely that the same mechanisms are at work that have been extensively studied in the context of solar wind interaction with cometary atmospheres (Brinca, 1991; Tsurutani, 1991) to explain the origin of waves at the water group pick-up ion gyrofrequency. It is known from these studies that ring-beam distributions, established by heavy pick-up ions, can drive several solar wind plasma modes unstable, preferentially low-frequency MHD waves at the gyrofrequency of the pick-up ions.

According to the standard resonance condition $\omega-k v_{\|} \pm n \Omega_{i}=0$ ( $\omega$ wave frequency in the solar wind frame, $v_{\|}$pick up ion drift velocity along the ambient field, $\Omega_{i}$ pick-up ion gyrofrequency) and in case of a super-Alfvénic drift velocity $v_{\|}$, two low-frequency MHD modes can resonantly interact with the pickup population to produce amplification at $\Omega_{i}(n=1)$. These are a right-hand polarized co-propagating (in direction of $v_{\|}$) fast mode and a counter-propagating left-hand polarized Alfvén wave (Price et al., 1988). In both cases the pick-up ions sense the unstable wave electric field in their own frame as nearly coincident with their gyration motion, ensuring an intense wave-particle interaction. Due to an anomalous Doppler shift, the polarization of the right-hand fast mode is reversed 
to left-handed in the spacecraft ( $\approx$ pick-up ion) frame. The fast mode resonant instability has largely been considered as the origin of low-frequency waves at the water group ion gyrofrequency observed around comets. In case of a sub-Alfvénic drift velocity $v_{\|}$with a dominating ring character of the pick-up ion distribution, the left-hand polarized Alfvén/proton cyclotron mode turns to become the most interesting resonant mode. The above resonance mechanism, in spite of its attractiveness, is not the most likely candidate to explain the PCWs at Mars because analytical and numerical studies have in common shown that the presence of newly created ions in the solar wind support preferentially the amplification of parallel propagating MHD waves under most conditions of interest, inconsistent with the clearly observed obliquity of PCW propagation at Mars $\left(\theta_{k B} \approx 20^{\circ}\right)$. Thus, another possibilities to interpret the observations cannot be ruled out a priory.

\subsubsection{Ion-Whistler Instabilities}

It should be mentioned that electromagnetic ring beam and/or pure ion beam instabilities can also excite waves well above the proton cyclotron frequency in the whistler range of frequencies which may exhibit peak wave growth for oblique propagation. This mechanism may be relevant for PCW generation since the unstable whistler frequency in the solar wind frame is significantly down shifted in the spacecraft $(\approx$ pick-up ion) frame to a frequency near to the proton gyrofrequency. Ion-whistler instabilities have first pointed out in the early study of solar windcomet interaction by Wu and Davidson (1972), were later analyzed by Goldstein and Wong (1987), and have been studied to explain upstream whistlers at Earth (Wong and Goldstein, 1988). More recently, the ion-whistler instability has been applied to interpret the so-called Phobos events (Baumgärtel et al., 1998) and the generation of PCWs at the AMPTE Ba release (Sauer et al., 1999). Since the generated waves are shorter wavelength whistlers, electron magnetization is essential rather than the gyration motion of the ion species involved, thus sometimes they are denoted as electron/ion whistler instabilities (Akimoto and Gary, 1987). These instabilities are believed to contribute little to the momentum coupling between pick-up ions and the solar wind but they may dominate over the relatively long wavelength resonant MHD modes in situations where the interaction region is spatially confined (smaller than the gyroradius of the pick-up ions), as, for example, for weak comets, in the AMPTE experiment or in the solar wind interaction with the Phobos neutral gas torus. The mechanism of the ion-whistler instability becomes apparent in an idealized picture in which one realizes that newborn ions, immediately after creation, tend to behave like a cold ion beam which is not in general aligned with the ambient magnetic field. The beam has super-Alfvénic velocity in general and thus can resonantly interact with the whistler branch of the solar wind plasma. In the approximation of an unmagnetized ion beam, the resonance can only be an ' $n=0$ resonance' $\omega-\mathbf{k v}_{b} \approx 0$ in which the component of the beam velocity along the propagation direction meets the whistler phase velocity. In a dispersion diagram the instability thus arises near to the cross point of the whistler mode phase 
velocity with the beam velocity component along $\mathbf{k}$ where interchanges between whistler type modes and beam modes take place. This is illustrated in Figure 26(a) which depicts a solution of the fully kinetic electrodynamic dispersion relation for the solar wind plasma penetrated by a thin proton beam, demonstrating the very narrow unstable wave number region. Figure 26(b) displays in addition the growth rate, maximized over $k$, and other related quantities as function of the propagation angle $\theta$ relative to the ambient magnetic field. As seen from this figure, the relevant mode is stable for field-aligned propagation and acquires the highest growth rates for finite obliquity. In the observer frame (beam frame $\approx$ spacecraft frame) the waves are left-hand polarized and the frequency at peak growth is seen to vary around $\Omega_{p}$, which appears typical for fairly thin and relatively cold beams. Finite electron temperature and a small but finite spread in the beam velocity combine to quench the unstable $k$ 's to a very narrow region resulting in the generation of an almost monochromatic wave. The generated waves thus appear to address all the constraints coming from the PCW observations. Along this way, even the oxygen exosphere of Mars may contribute to the generation of PCWs. Since their group velocity in the beam frame is almost zero, the waves do not tend to escape from the generation region and significant amplification may occur even in a spatially limited area. This property favors the above instability especially to account for the presence of $\Omega_{p}$-fluctuations at the Phobos-Deimos events. One may argue against the ion-whistler instability as source of PCWs at Mars because of the assumption of an unmagnetized ion beam. This may be justified for heavy pick-up ions but is not a good approximation for pick-up protons since their gyroradius is not large compared to the spatial scale of the interaction region, which results in an increase of the velocity spread of the beam.

\subsection{3. $\Omega_{p}$-Oscillations Seen in Bi-Ion Fluid Simulations}

An appropriate alternative mechanism for explaining PCWs at Mars has been found in bi-ion fluid simulations of mass-loading (Szegö et al., 2000). The bi-ion fluid approach (Sauer et al., 1994) uses a macroscopic concept which incorporates essential effects of the electromagnetic coupling between two ion fluids and the electrons through the Lorentz force and the condition of charge neutrality. The magnetic field is frozen into the electrons, so that Hall-current effects are included and differential streaming between the ion species automatically arises. The model anticipates the same local bulk velocity for all ions of each of the species which is not automatically satisfied in case of mass loading of a plasma flow, where, in general, freshly generated ions (at rest) coexist in the same region with pick-up ions which were born upstream at earlier time. However, in the two limit cases of a large or a small spatial extent of the mass-loading region, compared to the gyration radius of the secondary ions, the model can be expected to work satisfactorily. As an example, Figure 27 illustrates the results of one of the simulation runs. A proton-electron plasma (solar wind) with an Alfvén Mach number $M_{\mathrm{A}}=5$ enters the simulation box in which a homogeneously distributed source injects protons at a 

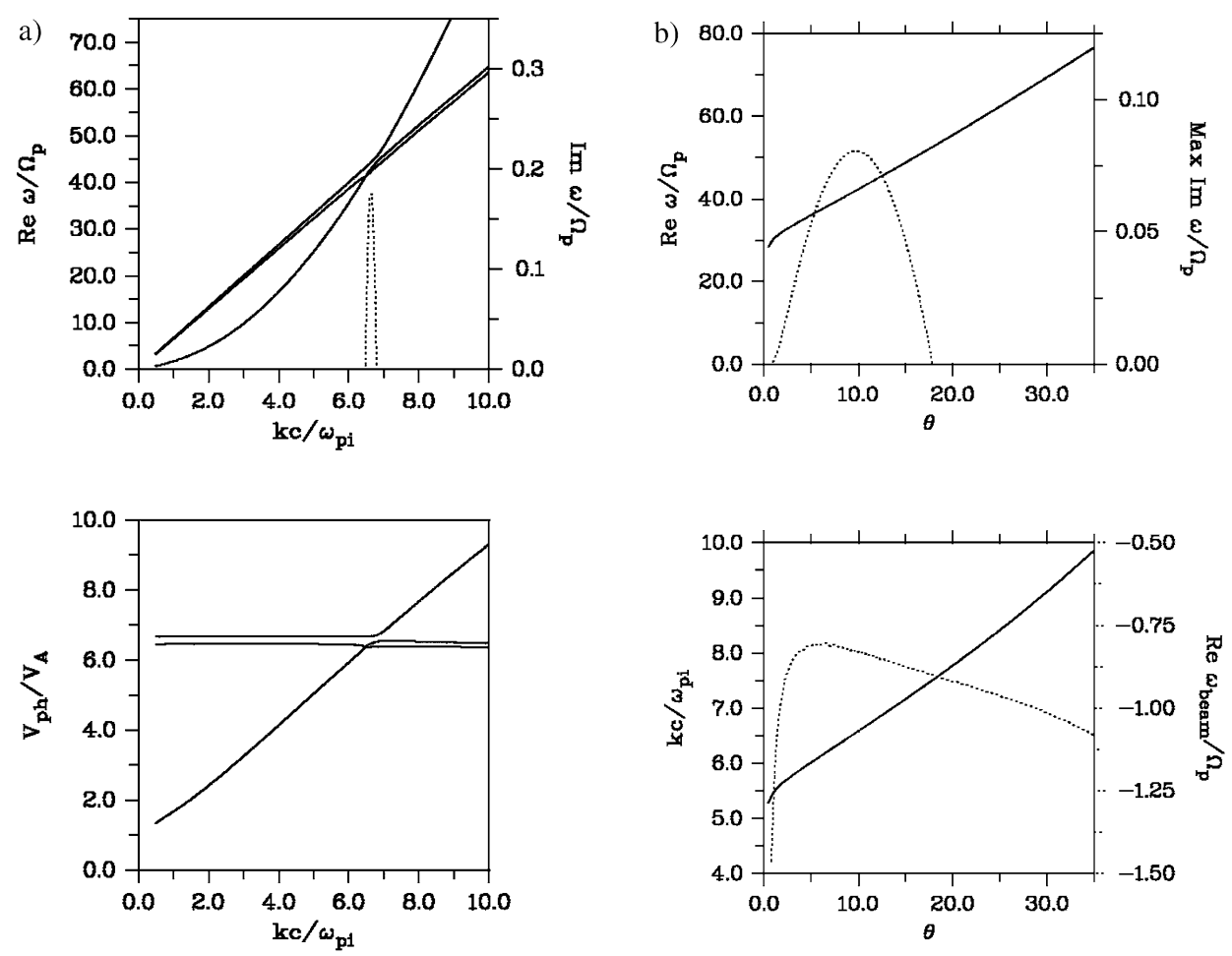

Figure 26. Warm plasma dispersion diagram for electromagnetic waves in the whistler range of frequencies in the solar wind plasma coexisting with a thin proton beam whose velocity vector $\mathbf{v}_{b}$ is $45^{\circ}$ inclined to the IMF and whose density is $0.125 \%$ of the ambient density. The wave vector $\mathbf{k}$ is in the $v_{b}-B_{0}$-plane and makes an angle $\theta$ to the magnetic field direction. (a) Top: real part (solid) and imaginary part (dotted) of the frequency normalized to $\Omega_{p}$ versus wave number. Bottom: phase velocity normalized to the $v_{\mathrm{A}}$ versus wavenumber. The beam frame frequency varies within the unstable $k$ region between $0.4 \Omega_{p}$ and $1.1 \Omega_{p}$. Parameters: $\theta=10^{\circ}, v_{b}=8 v_{\mathrm{A}}, \beta_{e}=0.5, \beta_{i}=1$, $\beta_{b}=0.01$. (b) Top: growth rate, maximized over $k$ (dotted), and corresponding real frequency (solid) versus propagation angle $\theta$ relative to the magnetic field. Bottom: wave number and beam frame frequency for peak growth versus $\theta$. Same parameters as in (a), except $\beta_{b}=0.02$ (Baumgärtel et al., 1998).

certain rate. After running the simulation a sufficiently long time, a quasi-stationary state is achieved which is shown in Figure 27. The most noticeable feature is seen in the spatial profiles of the magnetic field components $B_{y}$ and $B_{z}$ which indicate the presence of coherent waves. Their frequency can easily be calculated from the $x-t$-variation of $B_{z}$ shown on top of Figure 27 and is found to be slightly below the proton cyclotron frequency $\left(\approx 0.9 \Omega_{p}\right)$ in the observer system. The relative velocity between the core protons and the pick-up protons is gradually reduced along the simulation box and adjusted to a value $\Delta_{v} \approx 2 v_{\mathrm{A}}$ in a self-consistent way by the electromagnetic coupling between the two ion species. It is suggested that the presence of the rather coherent $\Omega_{p}$ oscillations seen in the simulations can be traced back to peculiarities in the dispersion characteristics of bi-ion plasma. As 


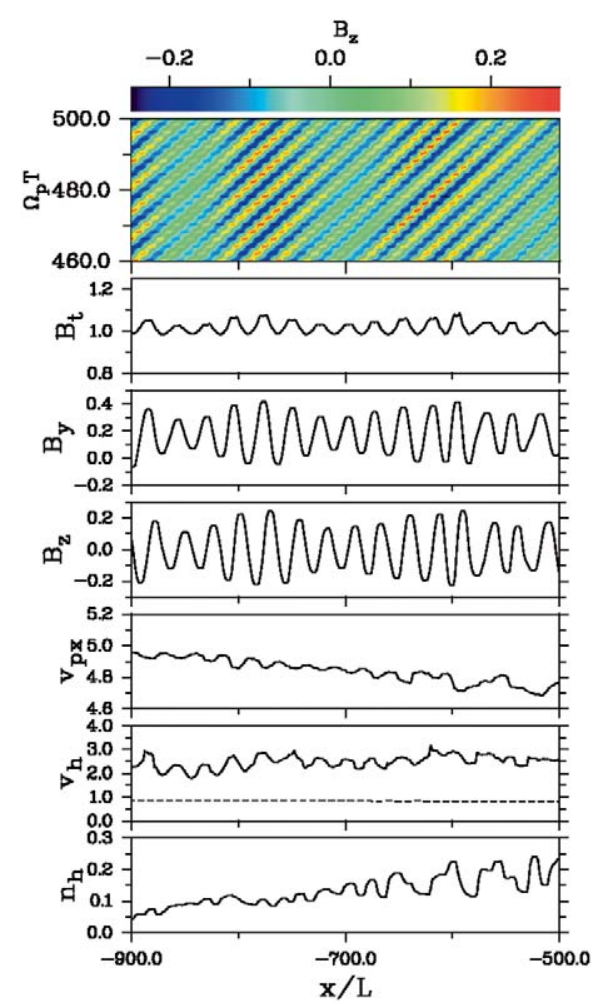

Figure 27. Bi-ion fluid simulation of solar wind mass loading by protons from an external source. The magnetic field is inclined to the $x$-axis by $10^{\circ}$. In the six panels (from bottom to top) the density and velocity of pick-up protons, the solar wind velocity, both components of the magnetic field $\left(B_{z}\right.$, $B_{y}$ ) and the magnitude $B_{t}$ are shown at $\Omega_{p} t=500$. The diagram on top displays the $x-t$-variation of $B_{z}$ which clearly indicates the presence of coherent waves with frequency close to the proton cyclotron frequency $\Omega_{p}$ (Sauer and Dubinin, 2001).

is well-known, the addition of a second ion population into proton-electron plasma strongly modifies the dispersion of low-frequency waves by producing a new cutoff frequency and the appearance of new modes. Modes that 'intersect' in the $U-k$ space $(U=\omega / k$, phase velocity) may be split which is usually accompanied by mode conversion and the appearance of two adjacent wavenumbers for which phase and group velocity coincide. The splitting may lead to 'gaps' in the dispersion diagram which are understood as phase velocity intervals which are not accessible for any of the small amplitude wave types for any real wave number. It is known from the Hall-MHD model of single-ion plasma that such 'gaps' exist even in absence of a second ion species and that they represent the domain of existence for MHD solitons (Baumgärtel, 1999). The 'gaps' are occupied by evanescent stationary waves $\left(\sim \exp \left( \pm i k(x-U t), k^{2}<0\right)\right.$ which, including nonlinear effects, give rise to periodic nonlinear wave structures, especially genuine solitons, as a result of the balance between nonlinear steepening and dispersive effects. 


\subsubsection{Two-Ion Solitons ('Oscillitons')}

A mechanism that particularly accounts for the generation of coherent wave packets is provided by the capability of a two-ion plasma to allow for particular solitary wave structures. Whereas solitons or the elements of periodic nonlinear wave structures in single-ion plasma exhibit sech-type space profile, the presence of a second ion species may create a new type of solitary waves in which a shorter wavelength spatial oscillation is superimposed the conventional sech-type shape ('oscillitons') (Sauer et al., 2001). To examine such solitary wave structures, one has to start with linear dispersion analysis to identify parameter regions (propagation velocity, angle of propagation, relative drift velocity, temperatures of the ion populations), which satisfy the 'gap' condition. Subsequently the system of ordinary differential equations need be solved, to which the basic system is reduced after introducing the stationary wave ansatz, i.e., the requirement that all variables depend on space and time only in the combination $x-U t$. This procedure, however, does not address the question of stability of the solitary solution which has to be tested separately by numerical solutions of the fully nonlinear time- and space-dependent basic system. In two-ion plasma, 'gaps' may be found with a non-zero real part of the wave number, which is responsible for the periodic substructure of a solitary wave. In numerical studies on the space-time evolution of perturbations in proton plasma superimposed by a thin proton beam, 'oscillitons' appeared as a rather robust structure (Sauer et al., 2001, 2002a, b). Figure 28 shows two examples of a stationary wave consisting of a train of solitary wave packets in two-ion plasma. An observer in the plasma frame sees a time variation due to the relative motion of the structure as a whole. The waves are intrinsically left-hand polarized, are found for oblique propagation relative to the ambient field, and the small-scale variation is determined by the real part of the complex wave number within the 'gap'. The coherent oscillations near $\Omega_{p}$ seen in the mass loading simulations are consistent with the presence of such structures. It should be noted here, that 'oscillitons' can exist also in unstable two-proton plasmas (different proton temperatures, relative drift, etc.). The philosophy of interpretation of $\Omega_{p}$-oscillations near Mars in terms of 'oscillitons' is based on the combination of two circumstances: the presence of a potentially unstable two-ion plasma with relative drift or temperature anisotropy as free energy source and the occurrence of mode splitting producing a wavenumber for which phase and group velocity coincide. It is suggested, that in this peculiar reference frame the plasma tends to develop a stable stationary structure (like an 'oscilliton') as a result of a frequency selection process from a possibly weak broadband source. When the spatially periodic structure sweeps over an observer, a signal periodic in time is recorded. The numerical findings so far predict that for conditions realized near Mars only oblique propagation guarantees the presence of the required singular point $v_{p h}=v_{g r}$ in the dispersion diagram, further, that the frequency seen by the observer at rest is always near to $\Omega_{p}$ and that the waves are left-hand polarized. These properties qualify this mechanism as one of the favored candidates for the excitation of PCWs at Mars. 

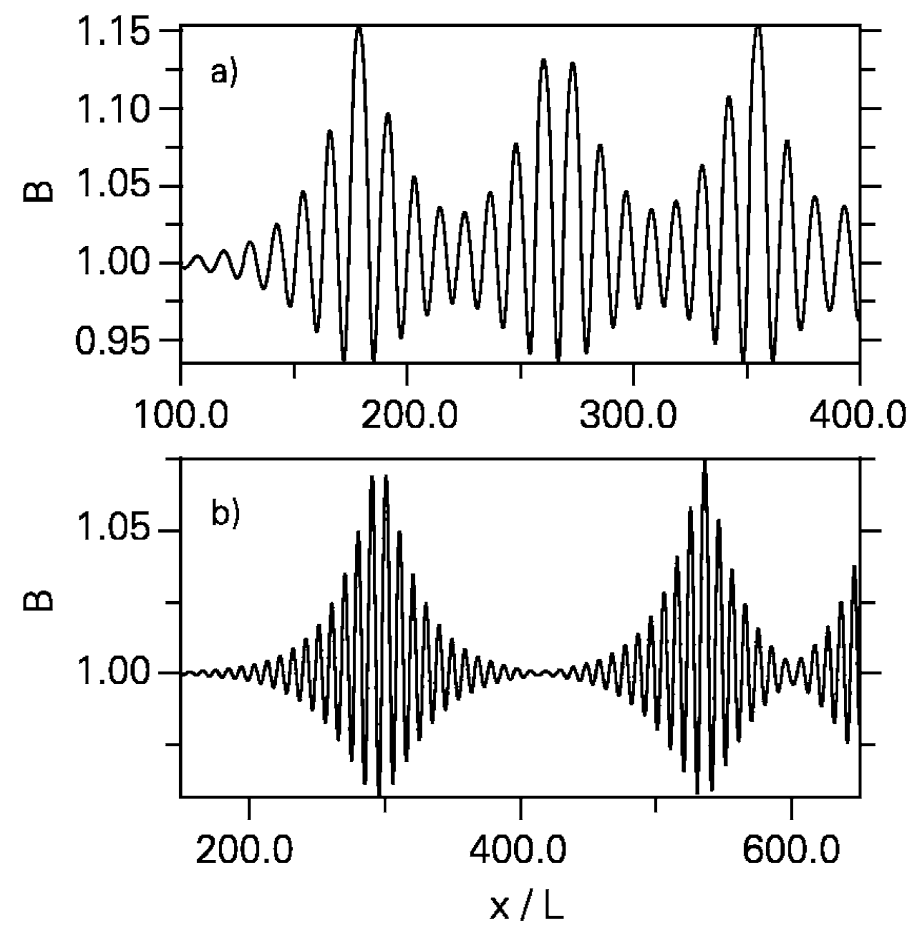

Figure 28. Two examples of bi-ion solitons with oscillating substructure ('oscillitons'). Top: cold proton plasma with an admixture of a cold heavier $\left(m_{h}=3 m_{p}\right)$ minor ion population $\left(n_{h}=0.25 n_{p}\right)$; Propagation velocity $U=0.95 v_{\mathrm{A}}$, propagation angle $\theta$ relative to the magnetic field $30^{\circ}$. Bottom: Warm proton plasma with an admixture of a second minor proton population with different temperature; $U=1.66, \theta=25^{\circ}, n_{p 2}=0.1 n_{p 1}, \beta_{e}=0.5, \beta_{p 1}=1.0, \beta_{p 2}=5.0$ (Sauer et al., 2001).

\subsubsection{Comparison Of The Theoretical Models}

The observed wave features of PCW's near Mars which must be addressed by any theoretical model for their interpretation include (1) frequency near to the local proton frequency, (2) left-hand polarization, (3) propagation at small to moderate angles to the ambient magnetic field and (4) spatial variation of wave intensity. There is general consensus that the PCW's can be attributed to the pick-up process of protons from the hydrogen corona of Mars. This is supported by the different amplitudes observed by Phobos and MGS which may be related to the different number densities of the neutral hydrogen atmosphere as a consequence of different cycles of solar activity. Phobos made measurements during solar maximum while MGS during solar minimum when the hydrogen density is almost two orders of magnitude higher. The conventional pick-up instabilities with peak growth at the gyrofrequency of the pick-up ions can likely be ruled out since they do not support the required generation of obliquely propagating waves. Both the high frequency whistler mechanism and the oscilliton model satisfy the requirements with respect to polarization and propagation direction, but they differ in the wavenumber of the 
waves thought to be responsible for the $\Omega_{p}$-signal. Whereas the high-frequency pick-up instability involves short wavelength $\left(k v_{p h} / \Omega_{p}>1\right)$ whistler waves, the oscilliton scheme predicts much longer wavelengths $\left.k v_{p h} / \Omega_{p}<1\right)$. Since no wave number estimate is provided by the observations, one cannot argue in terms of wavelength to discriminate between the two candidates. Also the question whether or not the PCW's are observed at their source cannot clearly be answered by the observations. The whistler instability predicts nearly group-standing waves in the observer frame which supports the idea of an observation in the source region; however, it is based on the assumption of unmagnetized beam ions which is not a good approximation for pick-up protons. The oscilliton model, on the other hand, does not predict group-standing waves in the observer frame but assumes the $\Omega_{p^{-}}$ signal to be produced by a spatial periodic wave structure, stationary in a reference frame with $v_{p h}=v_{g r}$, which is convected with (in general) super-Alfvénic velocity over the observer. The problem as to what extent details of the observed spatial variation of the PCW features (altitude variation of the wave intensity, existence of two regions of high wave concentration near the subsolar point and at the flanks, with slightly different wave properties) may help to judge the theoretical models, needs further investigations.

\section{Localized Disturbances in the Upstream Solar Wind}

As a preliminary remark it should be mentioned that a controversially discussed issue is addressed here which is still not well understood. Actually, there is no commonly accepted physical mechanism for interpretation which covers all the events. In several of the observations, some evidence is suggested that the Martian moons Phobos or Deimos act as source for the perturbations, either the bodies itself through their miniatmoshere, produced by weak outgassing or dust ejection, or via a gas/dust ring along their orbits. The first interpretation of disturbances as the interaction of Deimos with the solar wind was made by Bogdanov (1981). On the other hand, during the events the IMF was always in an orientation such as to place the spacecraft in the ion foreshock, i.e., in a region in which one would expect reflected ions, indicating a close relation to the Martian bow shock. The latter is further supported by similarities in the structure of certain events with the properties of hot flow anomalies (HFAs, hot diamagnetic cavities) observed upstream of Earth's bow shock, which suggest that comparable mechanisms may be at work upstream of Mars to produce the events as counterpart of the terrestrial HFAs without involving the presence of the Martian moons.

\subsection{Phobos/Deimos Events as Foreshock FEAtures}

It was discussed in the previous section that the reflection of pickup ions at the Martian bow shock could significantly enhance the wave activity in the foreshock 
region. A simple 'test particle approach' based on a reasonable model of the Martian hydrogen corona and the photoion motion in the prescribed electric and magnetic fields with electrostatic barrier at the bow shock provided the distribution of the proton number density along the spacecraft trajectories at which the PhobosDeimos events were observed. Significant enhancements of the plasma number density were found when the spacecraft occurred in the bunch of reflected from the bow shock exospheric protons. The position of this bunch is controlled by the interplanetary magnetic field and approximately coincides with the region occupied by the foreshock. Dubinin et al. (1995) suggested that 'overreflection' of the protons at the Martian bow shock due to a contribution of pickup ions may lead to a strong interaction between the solar wind and reflected protons and be responsible for the observed events. Figure 12 compares, for example, the model calculations and observations made on February 8, 1989 during the crossing of the hypothesized gas/dust Phobos torus. A reasonable agreement indicates that the appearance of the event may be related to such interaction. However it is important to note that the model is not self-consistent. The magnetic field configurations used for the calculation of ion trajectories were taken from the observations as the prescribed ones. On the other hand, the disturbed magnetic fields (the perturbations reach large amplitudes in the Phobos-Deimos events) may be caused by other sources, e.g., by the interplanetary disturbances, by ion currents of reflected particles and, at last, but not at least, by the solar wind - Phobos/Deimos/tori interaction.

\subsection{Hot Diamagnetic CAVities (HDC)}

It is known, for example, that when an interplanetary current sheet intersects the Earth's bow shock a structure termed a hot flow anomaly (HFA), or hot diamagnetic cavity, can form at the intersection point (Schwartz et al., 1985; Thomsen et al., 1986). Similar events with almost identical characteristics have been observed upstream of the Martian bow shock by MGS (Øieroset et al., 2001). An example of HFAs observed upstream of the Martian bow shock is shown in Figure 29(a), which displays magnetometer (MAG) and electron reflectometer (ER) observations from 13:13-14:42 UT on July 22, 1998. MGS crossed the bow shock from the magnetosheath side and entered the undisturbed solar wind at 14:06 UT. Ten minutes later (at 14:16 UT) the MAG/ER instrument recorded a $\sim 2$-min disturbance in both the energy flux (panel a) and the magnetic field magnitude and direction (panels $\mathrm{d}$, e, and f). Fourteen minutes after the first disturbance ended a second interval (at 14:32 UT) with similar characteristics was recorded. Figure 29(b) shows the first disturbance in detail. The event is characterized by a turbulent magnetic field (panel d) and enhanced electron temperature (panel c), while the density (panel b) remains at the same level as the undisturbed solar wind. The interval of turbulent field is flanked by large magnetic field and electron density enhancements. The inner and outer boundaries of this edge region are marked by the red and the black dashed lines, respectively. Øieroset et al. (2001) concluded that hot flow anomalies 

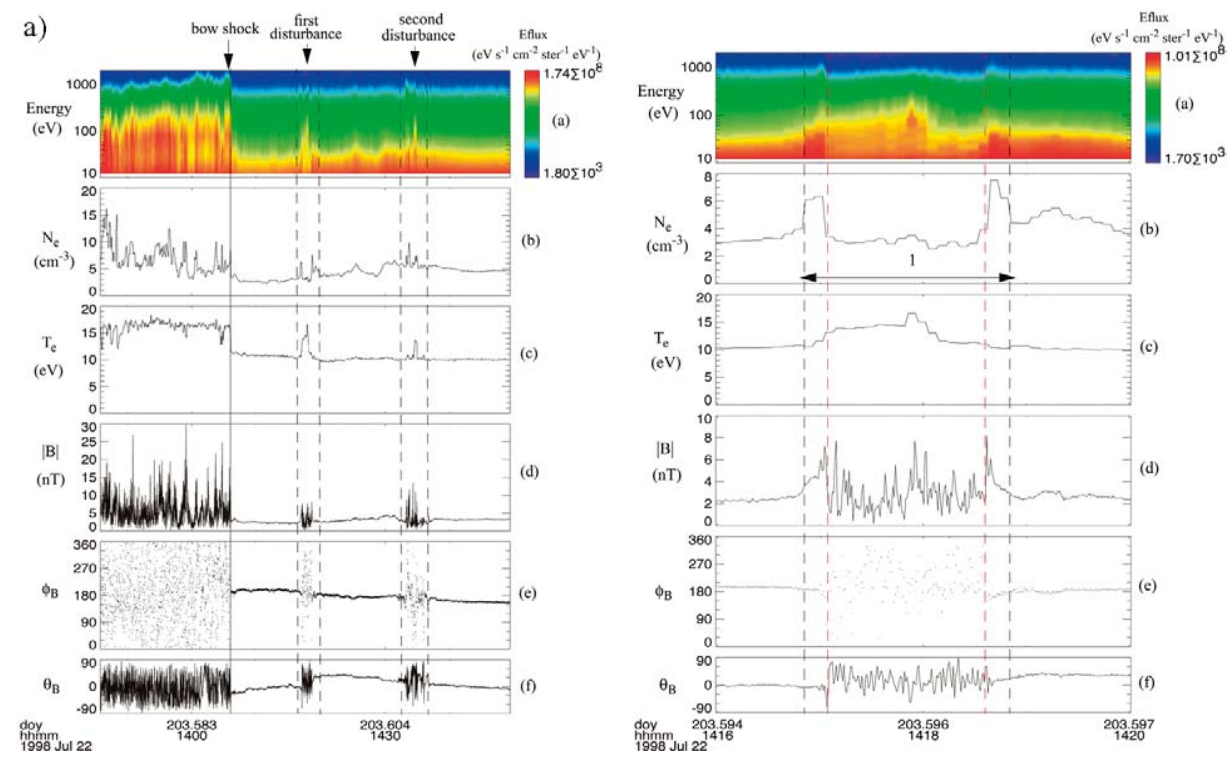

Figure 29. (a) MGS MAG/ER data from orbit (SPO) 443 on July 22, 1998. The electron energy spectrogram (energy flux) is shown in panel (a), while panel (b) and (c) show the electron density and temperature, respectively, both obtained by Maxwellian fitting of the electron spectra. The magnetic field observations are given in panels (d)-(f) as the magnetic field magnitude, the phi angle, and the theta angle. (b) Zoom-in of the first disturbance in (a). The hot central region is marked by the red dashed lines while the black dashed lines mark the outer boundaries of the edge regions. From Øieroset et al. (2001).

(HFAs) are a likely explanation for the events in Figure 29. They reached this conclusion based on the close similarity between the MGS events and HFAs observed upstream of the Earth's bow shock. For the Earth's case HFAs are believed to be caused by interplanetary current sheets interacting with the bow shock (Schwartz et al., 1985, 2000; Thomas et al., 1991). Øieroset et al. (2001) suggested that the same process could generate HFAs upstream of Mars. The presence of flow deflection, typical of terrestrial HFAs, cannot be verified for the MGS events because of the 2D nature of the MGS electron measurements.

A cartoon showing the generation of an HFA by an interplanetary current sheet interacting with the Martian bow shock is shown in Figure 30. A hot plasma region form outside the bow shock when ions initially reflected at the bow shock are channeled back along the current sheet by the motional electric field, which is directed toward the current sheet on both sides (Thomas et al., 1991; Lin, 1997). The high temperature region then expands, which leads to a decrease in the density and magnetic field magnitude inside this region, while the incoming solar wind is deflected with weak shock waves.

Before reaching their conclusion Øieroset et al. (2001) considered several candidate causes for the disturbances described above: (1) multiple bow shock cross- 


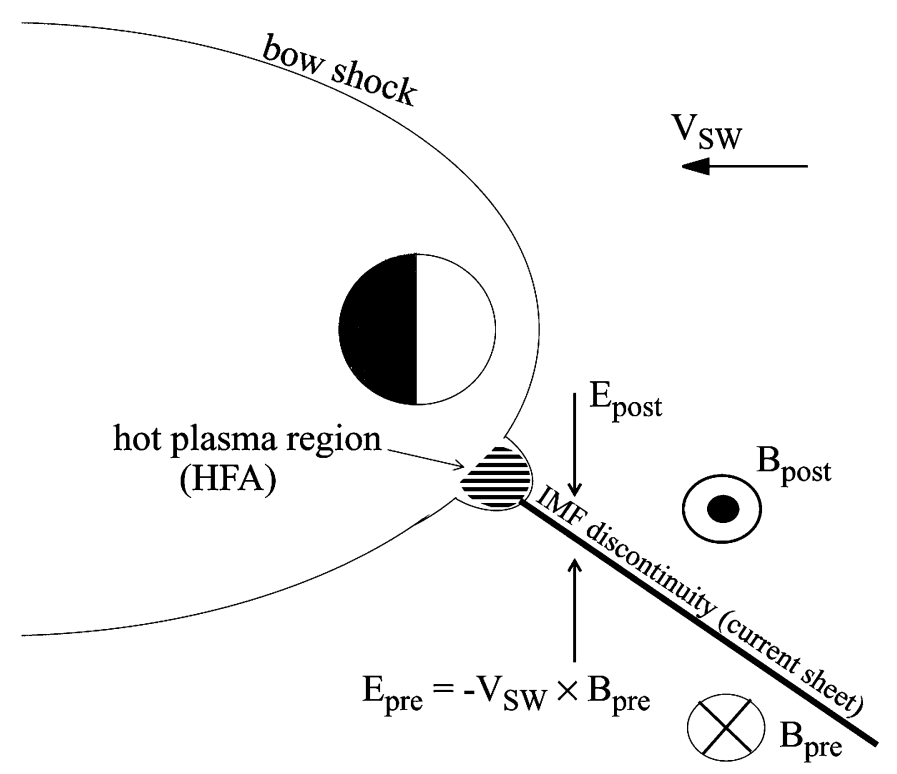

Figure 30. Cartoon showing the interaction of an interplanetary current sheet with the Martian bow shock. A hot flow anomaly (HFA) can form at the intersection point between the current sheet and the bow shock (figure adapted from Schwartz et al., 2000).

ings, (2) foreshock turbulence, and (3) outgassing and/or dust escape from the Martian moons. Figure 31 shows the location of the disturbances displayed in Figure 29 along the MGS orbit, as well as the Phobos and Deimos orbits and the location of the moons at the time of the events. The MGS spacecraft was located downstream of Deimos at the time when the electron and magnetic field disturbances were recorded. During the first disturbance MGS was located 9 degrees off the Deimos-Sun line, and for the second disturbance the spacecraft was 13 degrees off. Solar wind disturbances observed by the Phobos- 2 spacecraft have been suggested to be signatures of a Deimos Mach cone (Sauer et al., 1995b). Although the geometry for the MGS events suggests a causal relationship with the Deimos location, Øieroset et al. (2001) argued that other features of the observations are not consistent with such an interpretation. In particular, in a Mach cone crossing one would not expect the temperature enhancement to be flanked by a layer of magnetic field magnitude and density enhancements. On the contrary, for a Mach cone crossing the density, temperature, and magnetic field magnitude are expected to increase simultaneously (e.g., Sauer et al., 1995b). Øieroset et al. (2001) concluded that HFAs were a more likely explanation for the MGS events.

\subsection{MOdELS OF MOON-RELATED EVENT INTERPRETATION (MRE)}

The discussion whether or not small celestial bodies such as magnetized asteroids, weakly outgassing planetary moons or clouds/trails of charged dust can interact 

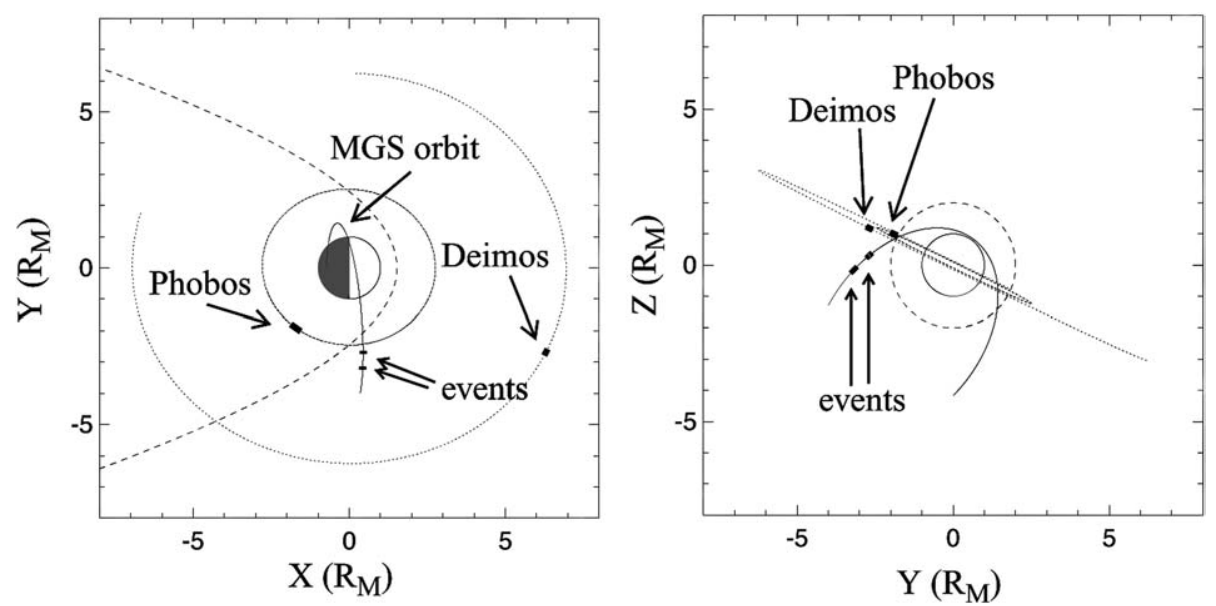

Figure 31. The MGS orbit in $x-y$ and $y-z$ sun synchronous ecliptic (SSE) coordinates (solid line). The two intervals of disturbances shown in Figure 1 are marked along the MGS orbit track. The orbits of Phobos and Deimos are shown with the dotted lines, and the positions of the moons at the time of the disturbances are also marked. In the SSE coordinate system X points from the center of the planet toward the Sun, the $\mathrm{Z}$ direction is normal to the ecliptic plane, pointing north, and $\mathrm{Y}$ is directed opposite to the planet's orbital velocity (Øieroset et al., 2001).
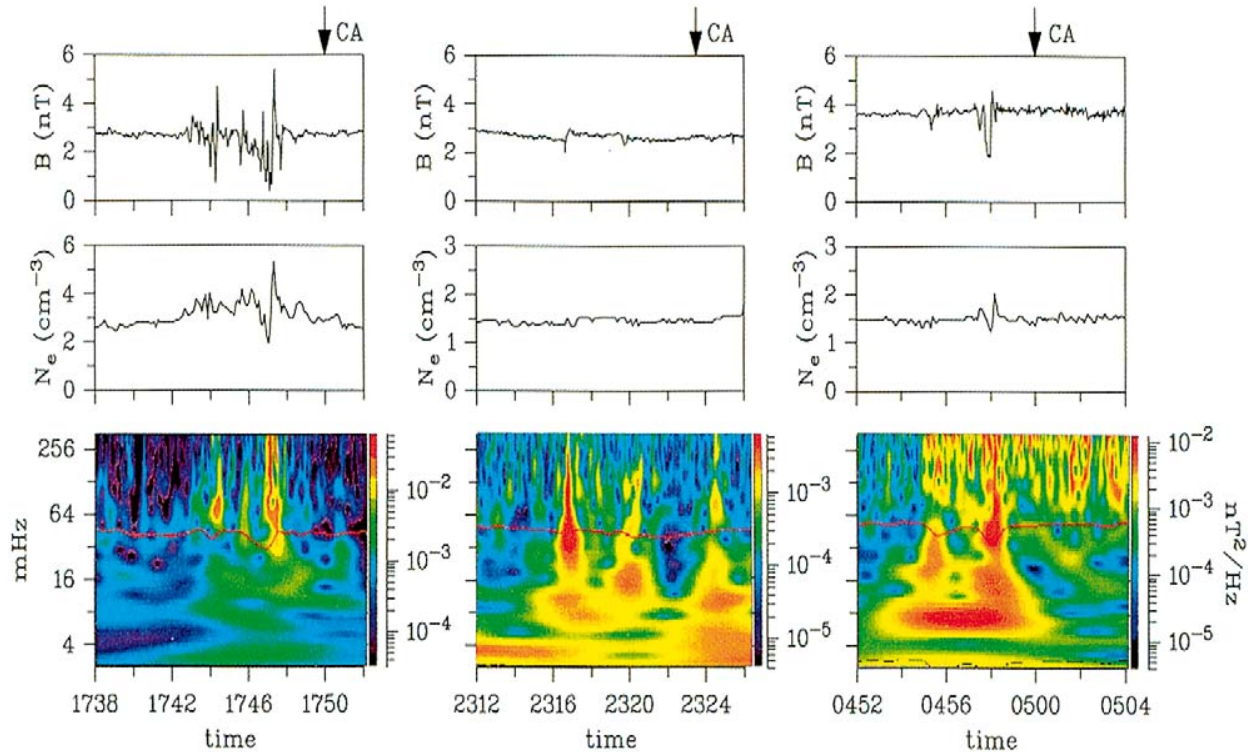

Figure 32. Phobos events observed by the Phobos-2 spacecraft during the first (a), the second (b) and the third (c) elliptical orbit (February 1, 4, 8, 1989) on just before crossing the Phobos orbit. The arrow indicates the time of closest approach. The bottom panel shows the low-frequency magnetic field power as a result of a wavelet analysis of the magnetic field turbulence. The proton cyclotron frequency is traced by a red line. (Baumgärtel et al., 1998). 
with the solar wind to produce observable effects, was initiated with a theoretical investigation of Greenstadt (1971) who predicted that a sufficiently magnetized asteroid may create interplanetary field perturbations that spacecraft magnetometers might detect. Observations by the Galileo satellite at its encounters with asteroids Gaspra and Ida and subsequent Hall-MHD simulations (Kivelson et al., 1993; Baumgärtel et al., 1994, 1997; Wang and Kivelson, 1996) supported an interpretation in terms of solar wind interaction with a magnetic dipole. The interest in this subject was considerably reinforced by remarkable Phobos-2 observations in 1989. During its first three elliptical orbits around Mars, the spacecraft observed significant, localized plasma and magnetic field perturbations upstream of the Martian bow shock. Several events were detected near to the crossing points of the spacecraft with the Phobos orbit whereas one of them occurred between the orbits of Phobos and Deimos ('mysterious' event). The events are seen as isolated perturbations in the magnitude of the interplanetary magnetic field, most pronounced at the edges of the perturbed region, accompanied by variations in the electron number density (Figure 32) in an otherwise relatively quiet solar wind. The events near the crossings of the Phobos orbit have been suggested as giving indirect evidence for the presence of either a gas ring or a dust ring along the Phobos orbit, and several theories have been worked out to explain these events in terms of solar wind interaction with a dust/gas torus (Dubinin et al., 1990, 1991, 1993; Krymski et al., 1992; Baumgärtel et al., 1998). This interpretation fails for the 'mysterious' event, for which, however, a particular position of Deimos relative to the spacecraft was realized which favours an interpretation in terms of a Mach cone crossing. Numerical simulations of the solar wind interaction with a weakly outgassing small body (Sauer et al., 1995b) confirmed the suggestion that the spacecraft crossed a fast wave Mach cone, with Deimos on top, about $15000 \mathrm{~km}$ downstream of it, as the event was recorded. We can therefore distinguish between two types of moon-related events: those which have been observed near to the orbits of Phobos or Deimos and those which are not related to the orbit of any of the moons but might be attributed to the presence of a Mach cone-like stationary wave pattern originating from the moons.

Figure 32 depicts magnetic field and electron density disturbances observed by the Phobos- 2 spacecraft during the first three elliptical orbits on just before crossing the Phobos orbit. Shown are records of the magnetic field amplitude, the electron density and the result of a wavelet analysis of the low-frequency magnetic field turbulence, with the proton cyclotron frequency indicated. A dust torus as well as a gas ring along the Phobos orbit has been proposed as source for the events. The existence of a Phobos dust torus was already postulated in 1971 by Sloter, but it has to date not been observed directly. The crucial parameter for any theory which ascribes the Phobos events to a dust torus is the magnitude of the total charge density established by the presence of charged dust grains of different size, because the solar wind responds to the dust only if its total charge density is not very small compared to the particle density of the solar wind protons. On the 
basis of a numerical simulation study on the dust ejecta from Phobos (Krivov and Hamilton, 1997), the total dust charge density was estimated to be about six orders of magnitude smaller than the solar wind proton density (Baumgärtel et al., 1998) which suggests that the Phobos dust torus can very likely be ruled out as source of the events. On the other hand, the possible presence of a gas ring with neutral number density exceeding that of the hot atomic oxygen background was pointed out by Ip and Banaskiewicz (1990) if a certain level of outgassing is maintained by the Phobos moon. The presence of a heavy ion population, generated by ionization from the neutral gas halo, with enhanced density near to the Phobos orbit, may give rise to electromagnetic ion beam-whistler instabilities which have been discussed in the context of proton cyclotron wave generation outside of the Martian bow shock earlier in this review. Unlike the scenario of solar wind mass loading from the hydrogen atmosphere of Mars, we are here confronted with a rather localized interaction region, with a size of only a fraction of the gyroradius of an exospheric ion, which justifies much better the approximation of unmagnetized beam ions. On the other hand, the higher mass of the beam ions $\left(m_{b}=16 m_{p}\right)$ requires a correspondingly higher beam density to reach growth rates comparable to those of a proton beam. According to a scenario for the neutral gas halo near Mars (Dubinin et al., 1991; Krymski et al., 1992) the oxygen ion density in the putative Phobos gas ring is of the order of $1 \%$ of the background proton density, as a result of a balance between ionization from the neutral gas torus and convective losses, on the basis of an outgassing rate of $10^{23} \mathrm{~s}^{-1}$ for Phobos. Warm plasma dispersion theory (Baumgärtel et al., 1998; Sauer et al., 1998a, 1999) predicts instability of the whistler mode supported by the solar wind plasma, for phase velocities near to the beam velocity projected on the whistler propagation direction. Peak growth is reached for oblique propagation $\left(\theta \approx 10^{\circ}\right)$ with real frequencies well above the proton cyclotron frequency and growth rates of the order of $0.1 \Omega_{p}$. The wavenumber near peak growth is $k c / \Omega_{p} \approx 5$, corresponding to a wavelength $\lambda \approx 200 \mathrm{~km}$. In the beam $(\approx$ observer) frame the frequency of the most unstable wave is downshifted to a value near to the proton gyrofrequency, consistent with the observed enhanced wave activity near to $\Omega_{p}$, seen in the bottom panel of Figure 32 . The group velocity of the fastest growing wave in the beam frame is almost zero which stretches the convective growth time, allowing for the unstable mode to acquire measurable amplitudes even in a limited area of interaction.

There are several mechanisms that could explain how a small body could perturb the solar wind (Herbert, 1993) and give rise to the generation of a wake in the surrounding plasma: cometary outgassing, inductive interaction of a conducting body, remnant magnetization, or a charged dust sphere around the body. Among them the comet-like interaction appears to be the most likely mechanism for Phobos and Deimos to act as obstacle because both moons are thought to outgas water, and thus, via ionization, may be capable to maintain a thin heavy ion cloud around them. According to a hypothesis of Fanale and Salvail (1998) the Martian moons contained a significant amount of water in their interior and have not lost 


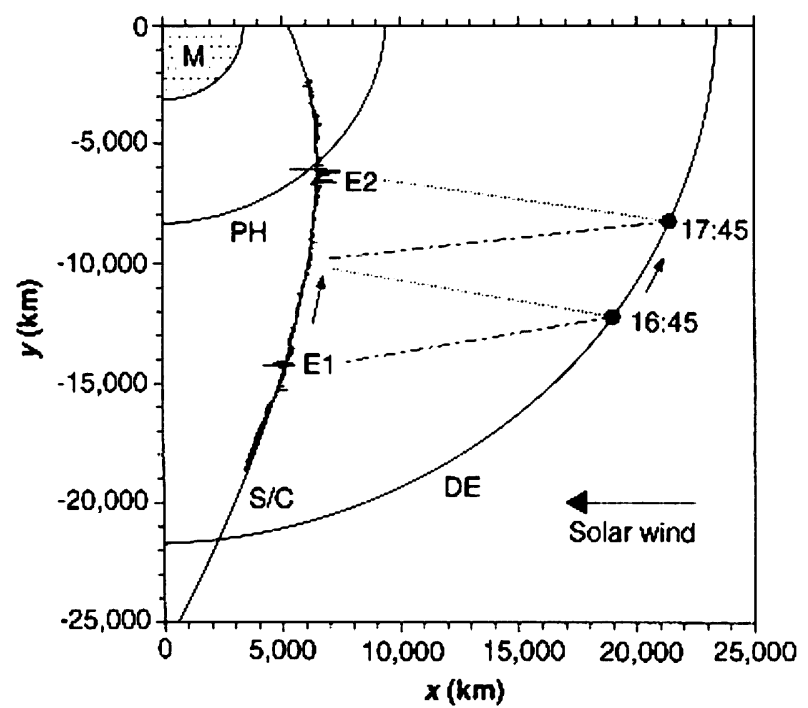

Figure 33. Location of the magnetic events E1 and E2 (February 1, 1989) in relation to the motion of Deimos. The spacecraft (S/C) trajectory and the orbits of the Martian moons Phobos (PH) and Deimos (DE) are plotted as solid lines. The curve along the spacecraft trajectory represents the relative deviation of the magnetic field amplitude from its mean value over three hours (resolution 10 s). The dotted and dashed-dotted lines illustrate that E1 and E2 may be related to spacecraft crossings of the left and right borders, respectively, of a Mach cone formed by the solar wind-Deimos interaction (Sauer et al., 1995).

it over geological times. The present water flux may reach values of $\sim 10^{23} \mathrm{~s}^{-1}$. It results in a gaseous envelope around the moons from which a plasma cloud is generated by ionization. It can be estimated that the above production rate is sufficient to maintain a heavy ion cloud with a peak density comparable with the number density of solar wind protons and a characteristic dimension of about twice the proton inertial length $(\approx 320 \mathrm{~km})$ as balance between newly produced ions and those carried away by the solar wind. Unlike the solar wind interaction with 'large' bodies such as magnetized or nonmagnetized planets, where a recognizable cavity against the solar wind ram pressure is maintained, the dominant feature in the solar wind response to a 'small' obstacle is wave generation. Therefore it seems appropriate to employ a linear response model which describes the interaction in terms of the stationary pattern of perturbations originating from the small body in the solar wind stream. The simplest and well-known wave pattern is the Mach cone formed behind a small body moving through air with supersonic velocity. The dispersionless waves continuously generated by the moving point-like source constructively interfere with each other to form a conical wave crest with the body on top, whereas elsewhere they destructively interfere to almost zero disturbance. This well-understood process may appear different where the ambient medium is anisotropic, such as the solar wind, so that waves propagate at different speeds in different directions. In the conventional MHD description, there are three different 
modes, each of which propagates with different speed for different angles with respect to the magnetic field, and each produces distinct bow/wake waves. The wave crest associated with the fast magnetosonic wave still resembles the standard Mach cone in that it appears only if the body's velocity exceeds the fast sound speed, although its geometry is no longer axisymmetric with respect to the flow direction. Wake waves supported by the Alfvén wave, however, are generated by any source speed and have little similarity to a conventional Mach cone; the Alfvén wave crest degenerates to a one-dimensional entity, called 'Alfvén wing' or 'Alfvén line'. These features are relaxed somewhat if the small-amplitude waves exhibit not only anisotropy but also dispersion, i.e., if they propagate at different speeds for different wavelengths. This arises in the solar wind if the wavelengths become comparable to or less than the proton inertial length $c / \Omega_{p i}$. In this case, the waves generated by the moving point source interfere to produce finite amplitude at more than a single wave crest surface. Thus the resulting stationary wave pattern may exhibit a rippled structure and may be thought of as a dispersed generalized Mach cone. The Mach cone philosophy has first been applied to the interpretation of events observed by Phobos-2 by Sauer et al. (1995b). Figure 33 illustrates the location of the 'mysterious' event (E1) and another event E2 near to the Phobos orbit (event (a) in Figure 32) in relation to the motion of Deimos. The spacecraft position relative to Deimos suggests that the events E1 and E2 might be the result of crossings of a fast wave Mach cone emanating from Deimos. The magnetosonic Mach number $v_{s w} /\left(v_{\mathrm{A}}^{2}+v_{S}^{2}\right)^{1 / 2}\left(v_{\mathrm{A}}\right.$ is the Alfvén velocity, $v_{s}$ is the acoustic velocity) was about 9 , corresponding to an opening angle for the Mach cone of about $13^{\circ}$, with little deviation from axisymmetry with respect to the flow direction. Because the satellite moved faster than Deimos by about $1 \mathrm{~km} \mathrm{~s}^{-1}$, it crossed the cone twice, inbound and outbound. Thereby the most pronounced event E2, which exhibits a rather complicated structure, appears to come about as a superposition of perturbations resulting from both the Phobos torus and the Deimos Mach cone. The diameter of the cone along the spacecraft orbit is about $4000 \mathrm{~km}$ and the spatial extent of the events is of the order of 300-500 km when considered as stationary entities. To account for nonlinear effects in the Mach cone evolution, simulations of the interaction have been carried out on the basis of the fully nonlinear MHD equations (Sauer et al., 1995b). Figure 34 depicts the result of a run which describes the stationary solar wind wake perturbations behind an excess charge cloud represented by a cloud of immobile ions with a size of $\approx 300 \mathrm{~km}$ for a more realistic Mach number. The formation of Mach cones is clearly seen. In addition to the observations in the solar wind Dubinin et al. (1991) have found the distinct variations in the magnetic field and plasma when the spacecraft, being in the magnetosheath, occurred in the Phobos-moon tail. The draping features were well correlated to the spacecraft position in the moon frame. Although similar perturbations may be also caused by a dense detached 'cloud' of planetary plasma, the bi-ion MHD simulations confirm that the disturbances generated by the Mach cone behind the moon can propagate downstream the bow shock (Figure 35). 


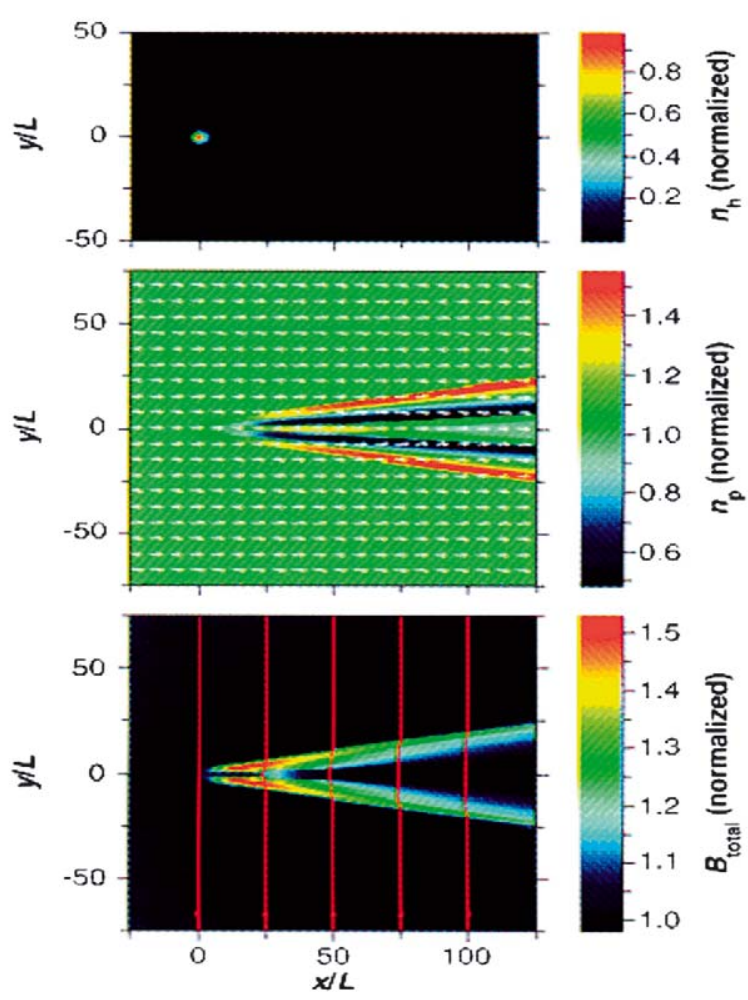

Figure 34. Solar wind interaction $\left(M_{f}=9\right.$, IMF perpendicular to the flow direction) with an immobile ion cloud of diameter $\approx 300 \mathrm{~km}$ calculated from the fully nonlinear MHD equations in 2-D approximation. Density and magnetic perturbations are seen to be concentrated along Mach cones. Arrows (middle panel) indicate the flow direction, red lines (bottom panel) correspond to IMF field lines (Sauer and Dubinin, 2001).

However, the direct relevance of the Mach cone pictures to the observed events is questionable, at least when only the moons itself and their plasma envelope is taken into consideration. Since the size of the Martian moons is of the order of $10 \mathrm{~km}$, the expected effective obstacle cross section does hardly exceed the proton inertial length (in the solar wind of the same order as the thermal proton gyroradius). In this case, the wake produced by the obstacle is dominated by the generation of short wavelength whistler waves and results in a dispersed Mach cone without long-ranging wings and separated magnetic and density perturbations.

On the final stage of the Phobos-2 mission the spacecraft was settled on the quasi-synchronous with the Phobos-Moon orbit. The spacecraft periodically approached the moon to the distance of $200 \mathrm{~km}$ and went away to the distance of $400 \mathrm{~km}$. The close encounters in the solar wind repeated every $\sim 8$ hours. The magnetic field measurements with time resolution of $45 \mathrm{~s}$ were made on $11 \mathrm{en-}$ counters. Figure 36 shows data for 8 closest approaches (CAs) (two orbits were not included because of gaps in the data). The distinct feature of the most CAs is 


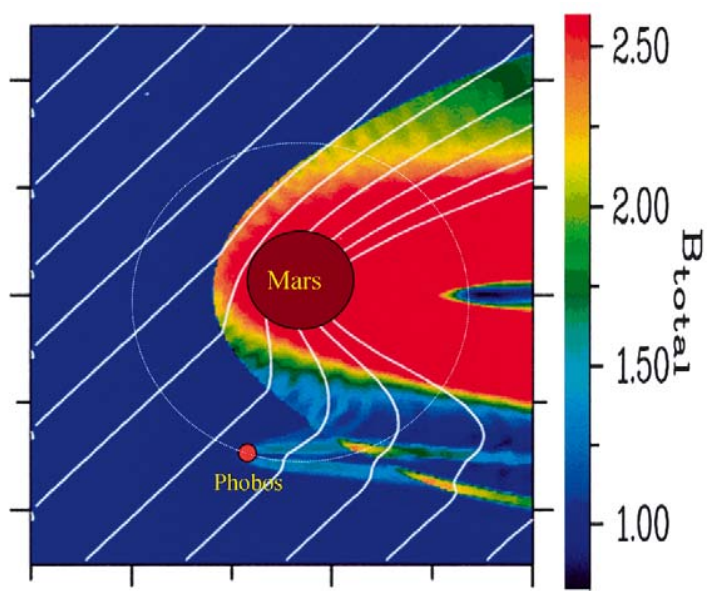

Figure 35. Solar wind interaction with the Phobos-moon and Mars calculated from the fully nonlinear MHD equations in 2-D approximation. Distinct magnetic field perturbations in the wake of the moon propagate downstream the Martian bow shock (Sauer and Dubinin, 2001).
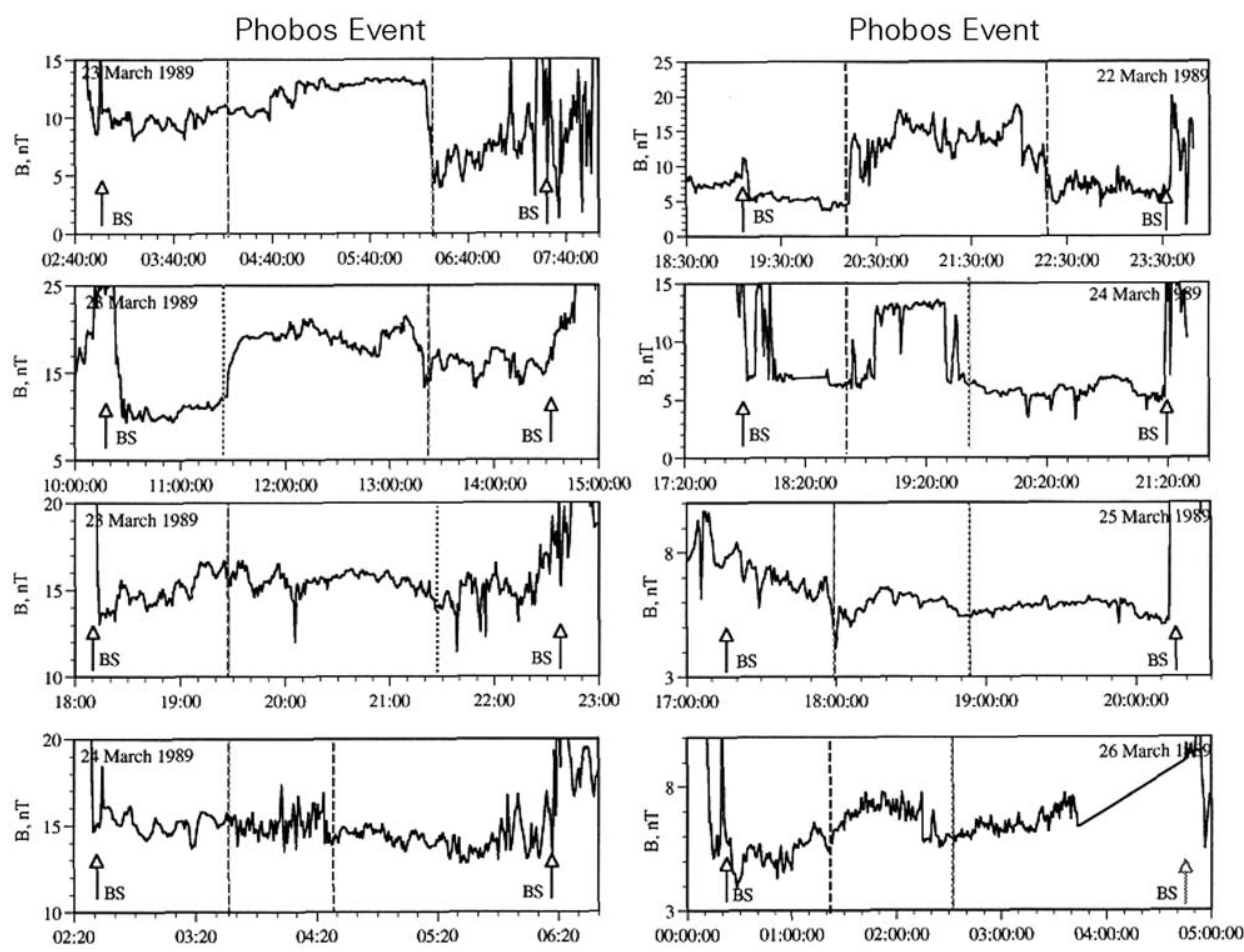

Figure 36. The set of the magnetic field measurements during the encounters with the Phobos moon on the final state of the Phobos-2 mission. Dotted curves show the intervals of the closest approaches when the distinct 'Phobos events' were observed (Sauer and Dubinin, 2001). 


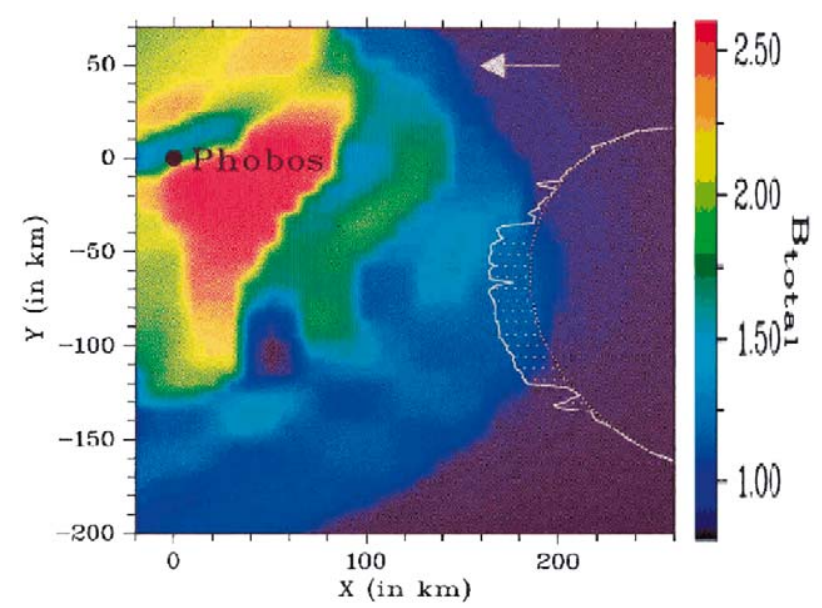

Figure 37. Composition of the magnetic field value in the neighborhood of the Phobos-moon from the bi-ion MHD simulations and the magnetic field strength along the spacecraft trajectory on the final state of the Phobos-2 mission. (Sauer and Dubinin, 2001).

a significant distortion of the magnetic field value. The perturbations range from shock-like enhancements to distinct strongly increased magnetic field fluctuations. Although some of the events were probably caused by the interplanetary shocks (e.g., at $\sim 21$ UT on 22 March or at $\sim 12$ UT on 23 March) or by outward motion of the bow shock (at $\sim 19$ UT on 24 March) the coincidence of all events with the closest approach is puzzling and therefore the interpretation in the terms of the moon events remains 'living'. Figure 37 shows the simulation results of the magnetic field disturbances in front of the outgassing moon. The white curve gives the magnetic field value along the spacecraft trajectory on March 24 in the moon reference frame. It is observed that the spacecraft could enter Phobos's 'minimagnetosphere'.

\section{Conclusions and Open Questions}

A few main points should be emphasised from the preceding sections. First, the bow shock at Mars is physically smaller than at all other solar system planets. This has implications for the plasma processes active at the shock and the amount of space available for the deceleration of the solar wind and for its collisionless heating via the wave-particle interaction processes in the downstream magnetosheath. At Mars, the thermalization of the ion populations is not expected to be complete in the dayside magnetosheath since the subsolar shock altitude is comparable to a typical solar wind proton gyroradius.

The Martian shock standoff distance is also larger relative to the size of the planet than at Venus. This was one argument often used in the past to infer the existence of a global dynamo magnetic field. We now know that Mars lacks such 
an internal magnetic field like Venus and present the same type of 'atmospheric' interaction with the solar wind as for active comets. This larger relative size of the shock comes mostly from the much more extended exosphere at Mars relative to the planet size, due to its weak gravity. This extension of the exosphere appears as the dimensioning parameter for the global plasma environment in such an interaction with the solar wind as it is for comets. This aspect is more widely discussed in a companion paper (Nagy et al., this issue).

The Martian shock is also much more variable on a day-to-day basis than at Venus, as has been clearly revealed by the MGS observations. However its mean location and shape seems very steady and is surprisingly at least only very weakly dependent on the solar cycle variations contrary to what is observed for Venus. The influence of the pickup ion dynamics creates a reliable but small asymmetry for the bow shock location along the direction of the convection electric field. The location of the shock is also locally influenced by the presence of the remnant crustal sources but only at a very small level.

The major cause for the day-to-day variability likely lays in the physical parameters of the shock itself. The Alfvén Mach number $M_{\mathrm{A}}$ is above 10 for typical upstream solar wind parameters at Mars and the magnetosonic Mach number (more suitable for the properties of such a fast-mode shock) is $M_{M_{s}} \sim 6$, typically. With such values of $M_{\mathrm{A}}$ and $M_{M s}$, the bow shock is extremely electromagnetically active. This is revealed both in the Phobos 2 and MGS observations. Moreover this is also a higher beta shock than at Venus. Another aspect comes from the possible effect of the curvature radius of the shock surface compared to the ion gyroradius. The major consequence is that the separation between quasi-parallel and quasiperpendicular shocks is not so relevant than for the Earth or Venus, which may have strong implications on the local processes at the bow shock such as particle reflection and acceleration.

A great variety of upstream waves have been detected at Mars, providing valuable clues about the physics at this planet. Mars differ from Earth and from Venus in that upstream waves are caused not only by particles reflected at the bow shock or leaked upstream through the shock, but waves are also generated by interactions between the solar wind and the exosphere. The most striking observation to that respect is the existence of highly coherent waves with very large amplitude at the cyclotron proton frequency.

Several outstanding questions should be addressed in future analyses and observations. What processes account for the differences between the shocks at Venus and Mars? If mass-loading makes the Martian shock standoff distance slightly larger than for Venus, why is there not a significant variation in this altitude with the solar cycle as there is at Venus? Why is the observed Martian bow shock not as asymmetric as predicted from kinetic simulations? What more can we learn about the different particle populations (incident solar wind, reflected particles, shocked solar wind particles leaking from magnetosheath, exosphere) from upstream waves? About the observed strong local disturbances, how can we discrim- 
inate between the interpretations of interplanetary current sheet interaction with the shock and moon effects? Answer to these questions may come from continued analysis of existing data sets and theoretical investigations. Answers may also come from future observations made at Mars, such as those that will be made by Mars Express.

\section{Acknowledgements}

The authors thank the International Space Science Institute (ISSI), Bern, Switzerland, for providing support to the International Team 'Mars Magnetism and its Interaction with the Solar Wind'. This review was realised from the work of this team.

\section{References}

Acuña M. H., Connerney, J. E. P., Wasilewski, P., Lin, R. P., Anderson, K. A., Carlson, W., McFadden, C. J., Curtis, D. W., Rème, H., Cros, A., Medale, J. L., Sauvaud, J. A., d'Uston, C., Bauer, S. J., Cloutier, P., Mayhew, M. and Ness, N. F.: 1992, 'The Mars Observer Magnetic Fields Investigations', J. Geophys. Res. 97, 7799-7814.

Acuña, M. H., Connerney, J. E. P., Wasilewski, P., Lin, R. P., Anderson, K. A., Carlson, C. W., McFadden, J., Curtis, D. W., Mitchell, D., Rème, H., Mazelle, C., Sauvaud, J. A., d'Uston, C., Cros, A., Médale, J. L., Bauer, S. J., Cloutier, P., Mayhew, M., Winterhalter, D. and Ness, N. F.: 1998, 'Magnetic Field and Plasma Observations at Mars: Initial Results of the Mars Global Surveyor Mission', Science 279, 1676-1680.

Acuña, M. H., Connerney, J. E. P., Ness, N. F., Lin, R. P., Mitchell, D., Carlson, C. W., McFadden, J., Anderson, K. A., Rème, H., Mazelle, C., Vignes, D., Wasilewski, P. and Cloutier, P.: 1999, 'Global Distribution of Crustal Magnetization Discovered by the Mars Global Surveyor MAG/ER Experiment', Science 284, 790-793.

Acuña, M. H., Connerney, J. E. P., Wasilewski, P., Lin, R. P., Mitchell, D., Anderson, K. A., Carlson, C. W., McFadden, J., Rème, H., Mazelle, C., Vignes, D., Bauer, S. J., Cloutier, P. and Ness, N. F.: 2001, 'Magnetic field of Mars: Summary of Results from the Aerobraking and Mapping Orbits', J. Geophys. Res. 106(10), 23403-23417.

Akimoto, K. and Gary, S. P.: 1987, 'Electron/ion Whistler Instabilities and Magnetic Noise Bursts', J. Geophys. Res. 92, 11209-11214.

Alexander, C. J. and Russell, C. T.: 1985, 'Solar Cycle Dependence of the Location of the Venus Bow Shock', Geophys. Res. Lett. 12, 369-371.

Alexander, C. J., Luhmann J. G. and Russell C. T.: 1986, 'Interplanetary Field Control of the Location of the Venus Bow Shock: Evidence for Comet-like Ion Pickup', Geophys. Res. Lett. 13, 917-920.

Axford, W. I.: 1991, 'A Commentary on Our Present Understanding of the Martian Magnetosphere', Planetary Space Sci. 39, 167.

Barabash S. and Lundin, R.: 1993, 'Reflected Ions Near Mars, Phobos-2 Observations', Geophys. Res. Lett. 20, 787.

Barabash, S., Dubinin, E., Pissarenko, N., Lundin, R. and Russell, C. T.: 1991, 'Picked-up Protons near Mars: Phobos Observations', Geophys. Res. Lett. 18, 1805-1808.

Barabash, S., Lundin, R. and Norberg, O.: 1993, 'Upstream Regions at Mars', Plasma Environment of Non-Magnetic Planets, COSPAR colloq. Ser. 4, in T. I. Combosi, (ed.), 285, Pergamon, New York. 
Baumgärtel, K.: 1999, 'Soliton Approach to Magnetic Holes', J. Geophys. Res. 104, 28295-28308.

Baumgärtel, K., Sauer, K. and Bogdanov, A.: 1994, 'A Magnetohydrodynamic Model Solar Wind Interaction with Asteroid Gaspra', Science, 263, 653-655.

Baumgärtel, K., Sauer, K., Story, T. R. and McKenzie, J. F.: 1997, 'Solar Wind Response to a Magnetized Asteroid: Linear Theory', Icarus, 129, 94-105.

Baumgärtel, K., Sauer, K., Dubinin, E., Tarasov, V. and Dougherty, M.: 1998, "Phobos events" Signatures of Solar Wind Interaction with a Gas Torus?', Earth, Planets and Space 50, 453462.

Bertucci, C., Mazelle, C., Crider, D. H., Vignes, D., Acuña, M. H., Mitchell, D. L., Lin, R. P., Connerney, J. E. P., Rème, H., Cloutier, P. A., Ness, N. F. and Winterhalter, D.: 2003a, 'Magnetic Field Draping Enhancement at the Martian Magnetic Pileup Boundary from Mars Global Surveyor Observations', Geophys. Res. Lett. 30(2), 1099.

Bertucci, C., Mazelle, C., Slavin, J. A., Russell, C. T. and Acuña, M. H.: 2003b, 'Magnetic Field Draping Enhancement at Venus: Evidence for a Magnetic Pileup Boundary', Geophys. Res. Lett. 30(17), 1876.

Bogdanov, A. V.: 1981, 'Mars Satellite Deimos Interaction with the Solar Wind and its Influence on Flow Around Mars', J. Geophys. Res, 86, 6926.

Bogdanov, A. V., Sauer, K., Baumgärtel, K. and Shrivastava, K.: 1996, 'Plasma Structures at Weakly Outgassing Comets - Results from Bi-ion fluid Analysis', Planetary Space Sci. 44, 519-528.

Bogdanov, T. and Motschmann, U.: 2002, 'From a weak to a strong comet-3D global hybrid simulation results', Earth, Moon and Planets, 90, 305-321, 2002.

Bonifazi C., Moreno, G., Lazarus, A. J. and Sullivan, J. D.: 1980, 'Deceleration of the Solar Wind in the Earth's Foreshock Region: ISSE-2 and IMP-8 Observations', J. Geophys. Res. 85, 6031.

Brain, D. A., Bagenal, F., Acuña, M. H., Connery, J. E. P., Crider, D. H., Mazelle, C., Mitchell, D. L. and Ness, N. F.: 2002, 'Observations of Low Frequency Electromagnetic Plasma Waves Upstream from the Martian shock', J. Geophys. Res. 107(A6), pp. 148-227 (10.1029/2000JA000416).

Brain, D. A., Bagenal, F., Acuña, M. H. and Connery, J. E. P.: 2003, 'Martian Magnetic Morphology: Contribution of Solar Wind and Crust', J. Geophys. Res. (in press).

Brecht, S. H.: 1990, 'Magnetic Asymmetries of Unmagnetized Planets', Geophys. Res. Lett. 17, 1243 $-1246$.

Brecht S. H.: 1997, 'Hybrid Simulations of the Magnetic Topology of Mars', J. Geophys. Res. 102, $4743-4750$.

Brecht, S. H.: 2002, 'Numerical Techniques Associated with Simulations of Solar Wind Interactions with Non-Magnetized Bodies', Comparative Aeronomy in the Solar System, AGU Press, New York (in press).

Brecht, S. H. and Ferrante, J. R.: 1991, 'Global Hybrid Simulation of Unmagnetized Planets: Comparison of Venus and Mars', J. Geophys. Res. 96, 11209-11220.

Brecht, S. H., Ferrante, J. R. and Luhmann, J. G.: 1993, 'Three-Dimensional Simulations of the Solar Wind Interaction with Mars', J. Geophys. Res. 98, 1345-1357.

Brinca, A.L.: 1991, 'Cometary Linear Instabilities: from Profusion to Prospective', Cometary Plasma Processes, in A. D. Johnstone (ed.), pp. 211-222, Geophysical Monograph 61, 211-222.

Cloutier, P. A., Chen, Y., Crider, D. H., Law, C. C., Walker, P. W., Acuña, M. H., Connerney, J. E. P., Lin, R. P., Anderson, K. A., Mitchell, D. L., Reme, H., Mazelle, C., Sauvaud, J. A., d'Uston, C., Vignes, D., Bauer, S. J. and Ness, N. F.: 1999, Venus-like Interaction of the Solar Wind with Mars', Geophys. Res. Lett. 26, 2685-2688.

Connerney, J. E. P., Acuña, M. H., Wasilewski, P. J., Ness, N. F., Rème, H., Mazelle, C., Vignes, D., Lin, R. P., Mitchell, D. L., Cloutier, P. A.,: 1999, 'Magnetic Lineation in the Ancien Crust of Mars', Science 284, 794-798.

Cravens, T. E., Shinagawa, H. and Luhmann, J. G.: 1997, in Stephen W. Bougher, D. M. Hunten and R. J. Philips (eds.), 'Magnetohydrodynamic Processes: Magnetic Fields in the Ionosphere of 
Venus, Venus II : Geology, Geophysics, Atmosphere, and Solar Wind Environment. University of Arizona Press, Tucson, AZ, p. 61.

Crider, D. H.: 1999, 'Evidence of Electron Impact Ionization in the Magnetic Pileup Boundary of Mars - Observations and modeling results, Ph.D. Thesis', Rice University, 109 pp.

Delva, M. and Dubinin, E.: 1998, 'Upstream ULF Fluctuations Near Mars', J. Geophys. Res. 103, 317.

Dubinin, E. M.: 1993, 'The Phobos and Deimos Effects', Adv. Space Res. 13, 271-130.

Dubinin, E. and Sauer, K.: 1999, 'The Martian Magnetosphere - Alaboratory for Bi-ion Plasma Investigations', Astrophys. Space Sci. 264, 273.

Dubinin E., Lundin, R., Koskinen, H. and Norberg, O.: 1993, 'Cold Ions at the Martian Bow Shock Phobos Observations', J. Geophys. Res. 98, 5617.

Dubinin, E. M., Lundin, R., Pissarenko, N. F., Barabash, S. V., Zakharov, A. V., Koskinen, H., Schwingenschuh, K. and Yeroshenko, Y. G.: 1990, 'Indirect Evidence for a Gas/Dust Torus Along the Phobos Orbit', Geophys. Res. Lett. 17, 861-864.

Dubinin, E. M., Pissarenko, N. F., Barabash, S. V., Zakharov, A. V., Lundin, R., Koskinen, H., Schwingenschuh, K. and Yeroshenko, Y. G.: 1991, 'Tails of Phobos and Deimos in the Solar Wind and in the Martian Magnetosphere', Planetary Space Sci. 39, 123-130.

Dubinin E. M., Obod, D., Pedersen, A. and Grard, R.: 1994, 'Mass-Loading Asymmetry in Upstream Region near Mars', Geophys. Res. Lett. 21, 2769.

Dubinin E. M., Obod, D., Lundin, R., Schwingenschuh, K. and Grard, R.: 1995, 'Some Features of the Martian Bow Shock', Adv. Space Res. 15(8/9), 423.

Dubinin, E., Sauer, K., Baumgärtel, K. and Srivastava, K.: 1998, 'Multiple Shocks at Mars', Earth Planets Space 50, 279-287.

Dubinin E. M., Sauer, K., Delva, M. et al.: 2000a, 'Multi-instrument Study of the Upstream Region near Mars: The Phobos-2 Observations', J. Geophys. Res. 105, 7557.

Dubinin E., Sauer, K. et al.: 2000b, 'Deceleration of the Solar Wind Upstream of the Martian Bow Shock. Mass-loading or Foreshock Features?', Adv. Space Res. 26(10), 1627.

Fanale, F. P. and Salvail, J. R.: 1989, 'Loss of Water from Phobos', Geophys. Res. Lett. 16, 287-290.

Fairfield, D. H.: 1974, 'Whistler Waves Observed Upstream from Collisionless Shocks', J. Geophys. Res. 79(10), 1368-1378.

Gleaves, D. G., Southwood, D. J., Dunlop, M. and Mier-Jedrzejowicz, W.: 1987, 'Low-Frequency Magnetic Wave Spectra Associated with the AMPTE Solar Wind Barium Release of 27 December 1984', Planetary Space Sci. 35, 493-500.

Goldstein, M. L. and Wong, H. K.: 1987, 'A Theory for Low-Frequency Waves Observed at Comet Giacobini-Zinner', J. Geophys. Res. 92, 4695-4700.

Grard, R., Klinge, D., Klimov, S., Savin, S. and Trotignon, J. G.: 1989, 'The Plasma Wave System on Phobos', J. Phys. E Sci. Instrum. 22, 888-894.

Grard, R., Nairn, C., Pedersen, A., Klimov, S., Savin, S., Skalsky, A. and Trotignon, J. G.: 1991, 'Plasma and Waves around Mars', Planetary Space Sci. 39, 89-98.

Grard, R., Skalsky, S. and Trotignon, J. G.: 1993, in T. I. Combosi (ed.), 'Selected Wave and Plasma Features of the Martian Environment', in Plasma Environment of non-Magnetic Planets, COSPAR Colloq. Ser. 4, 321-327.

Greenstadt, E. W.: 1971, 'Conditions for Magnetic Interaction of Asteroids with the Solar Wind, Icarus 14, 374-381.

Greenstadt, E. W., Le, G. and Strangeway, R. J.: 1995, 'ULF Waves in the Foreshock', Adv. Space Res. 15(8-9), 71-84.

Gringauz, K. I., Kurt, V. G., Moroz, V. I. and Shklovskii, I. S.: 1981, 'Results of Observations of Charged Particles Observed Out to $R=100000 \mathrm{~km}$, with the Aid of Charged-Particle Traps on Soviet Space Rockets', Soviet Astron. 4, 680.

Herbert, F.: 1993, 'Solar Wind Interaction with Asteroids', Adv. Space Res. 13(10), 249-258. 
Hopcroft, M. W. and Chapman, S. C., '2D Hybrid Simulations of the Solar Wind Interaction with a Small Scale Comet in High Mach Number Flows', Geophys. Res. Lett. 28(6), 1115.

Ip, W.-H.: 1988, 'On Hot Oxygen Corona at Mars', Icarus 76, 135.

Ip, W.-H.: 1990, 'The Fast Atomic Oxygen Corona Extent of Mars', Geophys. Res. Lett. 17, 22892292.

Ip, W.-H. and Banaszkiwicz, M.: 1990, 'On the Dust/Gas Tori of Phobos and Deimos', Geophys. Res. Lett. 17, 857.

Ip, W.-H.: 1992, 'Neutral Particle Environment of Mars: The Exosphere-Plasma Interaction Effects', Adv. Space Res. 12(9), 205.

Kim, J., Nagy, A. F., Fox, J. L. and Cravens, T. E.: 1998, 'Solar Cycle Variability of Hot Oxygen Corona at Mars', J. Geophys. Res. 103, 29339.

Kivelson, M. G., Bargatze, L. F., Khurana, K. K., Southwood, D. J., Walker, R. J., Coleman P. J. jr.: 1993, 'Magnetic Field Signatures Near Galileo's Closest Approach to Gaspra', Science 261, 331 -334 .

Kotova, G. et al.: 1997, 'Study of the Solar Wind Deceleration Upstream of the Martian Terminator Bow Shock', J. Geophys. Res. 102, 2165.

Krivov, V. A. and Hamilton, D. B.: 1997, 'Martian Dust Belts: Waiting for Discovery', Icarus 128, $335-353$.

Krymski, A. M., Breus, T. K., Dougherty, M. K., Southwood, D. J. and Axford, W. I.: 1992, 'The Electromagnetic Effects of the Solar Wind Interaction With the Phobos Neutral Gas Halo and Dust Torus', Planetary Space Sci. 40, 1033-1041.

Le, G., Blanco-Cano, X., Russell, C. T., Zhou, X.-W., Mozer, F., Trattner, K. J., Fuselier, S. A. and Anderson, B. J.: 2001, 'Electromagnetic Ion Cyclotron Waves in the High-Altitude Cusp: Polar Observations', J. Geophys. Res. 106, 19067-19079.

Lin, Y.: 1997, 'Generation of Anomalous Flows Near the Bow Shock by its Interaction with Interplanetary Discontinuities', J. Geophys. Res. 102, 24265.

Lipatov, A., Sauer, K. and Baumgärtel, K.: 1997, '2.5D Hybrid Code Simulation of the Solar Wind Interaction with Weak Comets and Related Objects', Planetary Space Sci. 20, 279-282.

Lipatov, A. S., Motschmann, U. and Bagdonat, T.: 2002, '3D Hybrid Simulations of the Interaction of the Solar Wind with a Weak Comet', Planetary Space Sci 50(4), 403-411.

Ma Y., Nagy, A. F., Hansen, K. C., DeZeeuw D. L. and Gombosi, T. I.: 2002, 'Three-Dimensional Multispecies MHD Studies of the Solar Wind Interaction with Mars in the Presence of Crustal Fields', J. Geophys. Res. 107(A10), 1282.

Mazelle, C. and Neubauer, F. M.: 1993, 'Discrete Wave Packets at the Proton Cyclotron Frequency at Comet P/Halley', Geophys. Res. Lett. 20, 153-156.

Mazelle, C., Rème, H., Vignes, D., Acuña, M. H., Brain, D., Connerney, J. E. P., Crider, D. H., Lin, R. P., Mitchell, D. L., Cloutier, P. A., Winterhalter, D. and Ness, N. F.: 2000, 'Nonlinear Low Frequency Waves Observations by Mars Global Surveyor', Eos Trans. AGU 81(48), Fall Meeting Suppl.

Mazelle, C., Bertucci, C., Sauer, K., Brain, D. A., Mitchell, D. L., Rème, H. and Acuña, M. H.: 2001, 'Properties of Upstream Waves at the Proton Cyclotron Frequency at Mars from MGS Observations', Eos Trans. AGU 82(47), Fall Meet. Suppl.

Mitchell, D. L., Lin, R. P., Mazelle, C., Rème, H., Cloutier, P. A., Connerney, J. E. P., Acuña, M. H. and Ness, N. F.: 2001, 'Probing Mars'Crustal Magnetic Field and Ionosphere with the MGS Electron Reflectometer', J. Geophys. Res. 106(E10), 23419-23428.

Mohlmann, D., Riedler, W., Rustenbach, J., Schwingenschuh, K., Kurths, J., Motschmann, U., Roatsch, T., Sauer, K. and Lichtenegger, H. T. M.: 1991, 'The Question of an Internal Martian Magnetic Field, Planetary Space Sci. 39, 83-88.

Moore, K. R., Thomas, V. A. and McComas, D. J.: 1991, 'Global Hybrid Simulation of the Solar Wind Interaction with Venus', J. Geophys. Res. 96, 7779-7791. 
Moses S. L., Coroniti, F. V. and Scarf, F. L.: 1988, 'Expectations for the Microphysics of the MarsSolar Wind Interaction', Geophys. Res. Lett. 15, 429-432.

Øieroset, M., Mitchell, D. L., Phan, T. D., Lin, R. P. and Acuña, M. H.: 2001, 'Hot Diamagnetic Cavities Upstream of the Martian Bow Shock', Geophys. Res. Lett. 28, 887.

Orlowski, D. S., Crawford, G. K. and Russell, C. T.: 1990, 'Upstream Waves at Mercury, Venus and Earth: Comparison of the Properties of One Hertz Waves', Geophys. Res. Lett. 17(12), $2293-$ 2296.

Orlowski, D. S., Russell, C. T., and Lepping, R. P.: 1992, 'Wave Phenomena in the Upstream Region of Saturn', J. Geophys. Res. 97(A12), 19187-19199.

Orlowski, D. S. and Russell, C. T.: 1995, 'Comparison of Properties of Upstream Whistlers at Different Planets', Adv. Space Res. 16(4), 137-141.

Pedersen, A., Nairn, C., Grard, R. and Schwingenschuh, K.: 1991, 'Deviation of Electron Densities from Differential Potential Measurements Upstream and Downstream of the Bow Shock and in the Magnetosphere of Mars', J. Geophys. Res. 96, 11243.

Price, C. P., Gaffey, J. D. and Dong, J. Q.: 1988, 'Excitations of Low-Frequency Hydromagnetic Waves by Freshly Created Ions in the Solar Wind', J. Geophys. Res. 93, 837-844.

Riedler W., et al.: 1989, 'Magnetic Fields Near Mars: First Results', Nature 341, 604.

Russell, C. T., Childers, D. D. and Coleman, P. J.: 1971, 'Ogo5 Observations of Upstream Waves in the Interplanetary Medium: Discrete Wave Packets', J. Geophys. Res. 76, 845-861.

Russell, C. T.: 1985, in B. Tsurutani and G. Stone (eds.), 'Planetary Bow shocks', Collisionless Shocks in the Heliosphere: Reviews of Current Research, Geophys. Monograph 35, 109, AGU.

Russell, C. T., Chou, E., Luhmann, J. G., Gazis, P., Brace, L. H. and Hoegy, W. R.: 1988, 'Solar and Interplanetary Control of the Location of the Venus Bow Shock', J. Geophys. Res 93, 54615469.

Russell, C. T., Luhmann, J. G., Schwingenschuh, K., Riedler, W. and Yeroshenko, Y.: 1990, 'Upstream Waves at Mars: Phobos Observations', Geophys. Res. Lett. 17, 897-900.

Sagdeev, R. Z., Shapiro, V. D., Shevchenko, V. I., Zacharov, A., Kiraly, P., Szego, K., Nagy, A. F. and Grard, R.: 1990, 'Wave Activity in the Neighborhood of the Bow Shock of Mars', Geophys. Res. Lett. 17, 893-896.

Sauer, K. and Dubinin, E.: 2000, 'The Nature of the Martian 'Obstacle Boundary', Adv. Space Res. 26, $1633-1637$.

Sauer, K., Bogdanov, A. and Baumgärtel, K.: 1994, 'Evidence of an Ion Composition Boundary (Protonopause) in Bi-ion Fluid Simulations of Solar Wind Mass Loading', Geophys. Res. Lett. 21, 2255-2258.

Sauer, K., Bogdanov, A. and Baumgärtel, K.: 1995a, 'The Protonopause - an Ion Composition Coundary in the Magnetosheath of Comets, Venus and Mars', Adv. Space Res. 16, 153-156.

Sauer, K., Dubinin, E., Baumgärtel, K., Bogdanov, A.: 1995b, 'Deimos: An obstacle to the Solar Wind', Science 269, 1075.

Sauer, K., Dubinin, E. and Baumgärtel, K.: 1997, 'Bi-ion Structuring in the Magnetosheath of Mars. Theoretical Modeling'. Adv. Space Res. 20, 137-140.

Sauer K., Baumgärtel, K., Dubinin, E. and Tarasov, V.: 1998a, 'Low-Frequency Electromagnetic Waves and Instabilities within the Martian Bi-ion Plasma', Earth, Planets Space 50, 269-278.

Sauer, K., Dubinin, E. and Baumgärtel, K.: 1998b, 'Nonlinear MHD Waves and Discontinuities in the Martian Magnetosheath. Observations and 2D Bi-ion MHD Simulations', Earth Planets Space, 50, 793-801.

Sauer, K., Dubinin, E., Dunlop, M., Baumgärtel, K. and Tarasov, V.: 1999, 'Low-Frequency Electromagnetic Waves Near and Below the Proton Cyclotron Frequency at the AMPTE Ba Release: Relevance to Comets and Mars', J. Geophys. Res. 104, 6763-6771.

Sauer, K., and Dubinin, E.: 2003, 'The Magnetic Pile-Up Boundary at Mars and Related Ion Dynamics: Observations and Simulations', Geophys. Res. Lett. (in press). 
Sauer, K., Dubinin, E. and McKenzie, J. F.: 2001, 'New Type of Soliton in Bi-ion Plasmas and Possible Implications', Geophys. Res. Lett. 28, 3589-3592.

Sauer, K., Dubinin, E. and McKenzie, J. F.: 2002a, 'Coherent Waves in Multi-Ion Plasmas', Physica Scripta T98, 52.

Sauer, K., Dubinin, E. and McKenzie, J. F.: 2002b, 'Wave Emission by Whistler Oscillitons: Application to Coherent Lion Roars', Geophys. Res. Lett. 29, 24, 2226, doi: 10.1029/2002 GLO15771, 2002.

Sauer, K., Dubinin, E., Phobos/Deimos events: observations and simulations, MPAE (Max-PlanckInstitut für Aeronomie) - Report: 3 - 2001.

Schwartz, S. J. et al.: 1985, 'An Active Current Sheet in the Solar Wind', Nature 318, 269.

Schwartz, S. J. et al.: 2000, 'Conditions for the Formation of Hot Flow Anomalies at Earth's Bow Shock', J. Geophys. Res. 105, 12639.

Schwingenschuh, K., Riedler, W., Lichtennegger, H., Yeroshenko, Y., Sauer, K, Luhmann, J. G., Ong, M. and Russell, C. T.: 1990, 'Martian Bow Shock: Phobos Observations', Geophys. Res. Lett. 17, 889.

Shimazu, H.: 1999, 'Three-Dimensional Hybrid Simulations of Magnetized Plasma Flow Around an Obstacle', Earth Planets Space 51, 383-393.

Shimazu, H.: 2001, 'Three-Dimensional Hybrid Simulation of Solar Wind Interaction With Unmagnetized Planets', J. Geophys. Res. 106, 8333-8342.

Skalsky, A., Grard, R., Klimov, S., Nairn, S. M. C., Trotignon, J. G., and Schwingenschuh, K.: 1992, 'The Martian Bow Shock: Wave Observations in the Upstream Region', J. Geophys. Res. 97, $2927-2933$.

Skalsky, A., Grard, R., Kiraly, P., Klimov, S., Kopanyi, V., Schwingenschuh, K. and Trotignon, J. G.: 1993, Simultaneous Plasma Wave and Electron Flux Observations Upstream of the Martian Bow Shock', Planetary Space Sci. 41, 183-188.

Skalsky A., Dubinin, E., Petrukovich, A., Sauer, K., Kiraly P. and Schwingenschuh, K.: 1998, 'Ion and Electron Heating at the Martian Bow Shock, Common for Bow Shocks or Not?', Earth Planets Space 50, 289.

Slavin, J. A., Elphic, R. C., Russell, C. T., Wolfe, J. H. and Intriligator, D. S.: 1979, 'Position and Shape of the Venus Bow Shock - Pioneer Venus Orbiter Observations', Geophys. Res. Lett. 6, 901-904.

Slavin J. A. and Holzer, R. E.: 1981, 'Solar Wind Flow About the Terrestrial Planets: 1. Modeling Bow Shock Position and shape', J. Geophys. Res. 86, 11401-11418.

Slavin J. A. and Holzer, R. E., 1982, 'The Solar Wind Interaction with Mars Revisited, J. Geophys. Res.87(B12), 10285-10296.

Slavin, J. A., Holzer, R. E. Spreiter, J. R. Stahara, S. S. and Chaussee, D. S.: 1983, 'Solar Wind Flow About the Terrestrial Planets. II - Comparison With Gas Dynamic Theory and Implications for Solar-Planetary Interactions', J. Geophys. Res. 88, 19-35.

Slavin, J. A., Schwingenschuh, K., Riedler, W. and Eroshenko, E.: 1991, 'The Solar Wind Interaction with Mars - Mariner 4, Mars 2, Mars 3, Mars 5 and PHOBOS 2 Observations of Bow Shock Position and Shape', J. Geophys. Res.96, 11235-11241.

Sloter, S.: 1971, 'The Dust Belts of Mars, Cornell Center for Radiophys. and Space Phys., Rep. No. 462.

Smith, E. J., Davis, L. Jr., Coleman, P. J. Jr. and Jones, D. E.: 1965, 'Magnetic Field Measurements Near Mars', Science 149, 1241-1242.

Spangler, S., Fey, A., Anderson, G. and Fuselier, S.: 1988, 'An Observational Study of MHD Waveinduced Density Fluctuations Upstream of the Earth's Bow Shock', J. Geophys. Res. 93, 845857.

Spreiter, J. R. and Stahara, S S.: 1980, 'A New Predictive Model for Determining Solar WindTerrestrial Planet Interactions’, J. Geophys. Res. 85, 6769-6777. 
Strangeway, R. J. and Crawford, G. K.: 1995, 'Comparison of Upstream Phenomena at Venus and Earth', Adv. Space Res. 16, (4), 125-136.

Szegö, K., Glassmeier, K.-H., Bingham, R., Bogdanov, A., Fischer, C., Haerendel, G., Brinca, A., Cravens, T., Dubinin, E., Sauer, K., Fisk, L., Gombosi, T., Schwadron, N., Isenberg, P., Lee, M., Mazelle, Ch., Möbius, E., Motschmann, U., Shapiro, V. D., Tsurutani, B. and Zang, G.: 2000, 'Physics of Massloaded Plasmas', Space Sci. Rev. 94(3/4).

Tatrallyay, M., Gevai, G. and Apathy, I. et al.: 1997, 'Magnetic Field Overshoots in the Martian Bow Shock', J. Geophys. Res. 102, 2157.

Thomas, V. A. and Brecht, S. H.: 1987, 'Angular Dependence of High Mach Number Plasma Interactions', J. Geophys. Res. $92,3175$.

Thomas, V. A. et al.: 1991, 'Hybrid Simulation of the Formation of a Hot Flow Anomaly', J. Geophys. Res. 96, 11625.

Thomsen, M. F. et al.: 1986, 'Hot, Diamagnetic Cavities Upstream from the Earth's Bow Shock', J. Geophys. Res. 91, 2961.

Trotignon, J. G., Hamelin, M., Grard, R., Pedersen, A., Klimov, S., Savin, S. and Skalsky, A.: 1991a, 'A Comparison of the Earth's and Mars' Bow Shocks Detected by the Phobos Plasma-Wave System', Planetary Space Sci. 39, 99-112.

Trotignon, J. G., Grard, R. and Klimov, S.: 1991b, 'Location of the Martian Bow Shock Measurements by the Plasma Wave System on Phobos 2', Geophys. Res. Lett. 18, 365-368.

Trotignon, J. G., Grard, R. and Savin, S.: 1991c, Plasma Wave System Measurements of the Martian Bow Shock from the Phobos 2 Spacecraft', J. Geophys. Res. 96, 11253-11264.

Trotignon, J. G., Skalsky, A., Grard, R., Nairn, C. and Klimov, S.: 1992, 'Electron Density in the Martian Foreshock as a By-product of the Electron Plasma Oscillation Observations', J. Geophys. Res. 97, 10831-10840.

Trotignon, J. G., Grard, R. and Skalsky, A.: 1993, 'Position and Shape of the Martian Bow Shock: the Phobos 2 Plasma Wave System Observations', Planetary Space Sci. 41, 189-198.

Trotignon, J. G., Trotignon, A., Dubinin, E., Skalsky, A., Grard, R. and Schwingenschuh, K.: 2000, 'Electron Plasma Waves Beyond the Mars' Bow Shock: the PWS/Phobos 2 Observations', Adv. Space Res. 26(10), 1619-1622.

Tsurutani, B. T.: 1991, in A.D. Johnstone (ed.), Comets: A Laboratory for Plasma Waves and Instabilities', Cometary Plasma Processes, pp. 189-209, Geophysical Monograph 61, 189-209, American Geophysical Union.

Tsurutani, B. T., Brinca, A. L., Buti, B., Smith, E. J., Thorne, R. M. and Matsumoto, H.: 1989, 'Magnetic Pulses with Duration Near the Local Proton Cyclotron Period: Comet Giacobini-Zinner', $J$. Geophys. Res. 94, 29-35.

Vaisberg, O. L. and Zelenyi, L. M.: 1984, 'Formation of the Plasma Mantle in the Venusian Magnetosphere', ICARUS 58, 412-430.

Vaisberg, O. L.: 1992a, 'The Solar Wind Interaction with Mars: a Review of Results from Previous Soviet Missions to Mars', Adv. Space Res. 12(9), 137-161.

Vaisberg, O. L.: 1992b, 'The Solar Wind Interaction with Mars: a Review of Results from Previous Soviet Missions to Mars, in: Venus and Mars: Atmospheres, Ionospheres, and Solar Wind Interactions, Proceedings of 1990 Chapman conference, Geophysical Monograph 66, pp. 311-326, American Geophysical Union.

Verigin, M. I., Gringauz, K. I. et al.: 1991, 'On the Problem of the Martian Atmosphere Dissipation: Phobos-2 TAUS Spectrometer Results', J. Geophys. Res. 96, 19315.

Verigin, M. I. et al.: 1993, 'The Dependence of the Martian Magnetopause and Bow Shock on Solar Wind Ram Pressure According to Phobos 2 TAUS Ion Spectrometer Seasurements', J. Geophys. Res. 98, 1303.

Verigin, M., Kotova, G., Shutte, N., Remizov, A., Szegö, K., Tátrallyay, M., Apáthy, I., Rosenbauer, H., Livi, S., Richter, A. K., Schwingenschuh, K., Zhang, T.-L. and Slavin, J.: 1997, 'Quantitative 
Model of the Martian Magnetopause Shape and its Variation with the Solar Wind Ram Pressure Based on Phobos 2 Observations', J. Geophys. Res. 102(A2), 2147-2156.

Verigin, M. I., Kotova, G. A., Remizov, A. P., Styazhkin, V. A., Schutte, N. M., Zhang, T.-L., Riedler, W., Rosenbauer, H., Szegö, K., Tatrallyay, M. and Schwingenschuh, K., 'Shape and Location of Planetary Bow Shocks', 1999 Cosmic Research 37(1), 34.

Vignes, D., Mazelle, C., Rème, H., Acuña, M. H., Connerney, J. E. P., Lin, R. P., Mitchell, D. L., Cloutier, P., Crider, D. H. and Ness, N.VF.: 2000, The Solar Wind Interaction with Mars: Locations and shapes of the Bow Shock and the Magnetic Pile-up Boundary from the Observations of the MAG/ER Experiment Onboard Mars Global Surveyor', Geophys. Res. Lett. 27(1), 49-52.

Vignes, D., Acuña, M. H., Connerney, J. E. P., Crider, D. H., Mazelle, C. and Rème, H.: 2002 'Factors Controlling the Location of the Bow Shock at Mars', Geophys. Res. Lett. 29(9), 42-1-42-4.

Wang, Z. and Kivelson, M. G.: 1996, 'Asteroid Interaction with Solar Wind', J. Geophys. Res. 101, 24479-24493.

Wong, H. K. and Goldstein, M. L.: 1988, 'Proton Beam Generation of Oblique Whistler Waves', J. Geophys. Res. 93, 4110-4114.

Wu, C. S. and Davidson, R. C.: 1972, 'Electromagnetic Instabilities Produced by Neutral-particle Ionization in Interplanetary Space', J. Geophys. Res. 77, 5399.

Zakharov, A. V.: 1992, 'The Plasma Environment of Mars: Phobos Mission Results. A 1990 status', Adv. Space Res. 12(9), 169-189.

Zelenyi, L. M. and Vaisberg, O. L.: 1985, in Buti (ed.), 'Venusian Interaction with the Solar Wind Plasma Flow as a Limiting Case of the Cometary Type Interaction', Advances of Space Plasma Physics World Scientific, Singapore, pp. 59-76.

Zhang, T.-L., Luhmann, J. G. and Russell, C. T.: 1990, 'The Solar Cycle Dependence of the Location and Shape of the Venus Bow Shock', J. Geophys. Res. 95, 14961-14967.

Zhang, T. L., Schwingenschuh, K., Lichtenegger, H., Riedler, W. and Russell, C. T.: 1991, 'Interplanetary Magnetic Field Control of the Mars Bow Shock - Evidence for Venuslike Interaction', J. Geophys. Res. 96, 11265-11269.

Zhang T. L., Schwingenschuh, K. and Russell, C. T.: 1995, 'A Study of the Solar Wind Deceleration in the Earth's Foreshock Region', Adv. Space Res. 15(8/9), 137.

Zhang T. L. et al.: 1997, 'Solar Sind Deceleration at Mars and Earth: A Comparison', Adv. Space Res. 15(2), 133. 